\title{
CONVERGENCE OF QUANTUM COHOMOLOGY BY QUANTUM LEFSCHETZ
}

\author{
HIROSHI IRITANI
}

\begin{abstract}
Quantum Lefschetz theorem by Coates and Givental 4 gives a relationship between the genus 0 Gromov-Witten theory of $X$ and the twisted theory by a line bundle $\mathcal{L}$ on $X$. We prove the convergence of the twisted theory under the assumption that the genus 0 theory for original $X$ converges. As a byproduct, we prove the semisimplicity and the Virasoro conjecture for the Gromov-Witten theories of (not necessarily Fano) projective toric manifolds.
\end{abstract}

\section{INTRODUCTION}

Quantum cohomology is a deformation of the ring structure of the ordinary cohomology. The structure constants of quantum cohomology are formal power series whose coefficients consist of Gromov-Witten invariants. We do not know a priori whether or not the structure constants are convergent. In this paper, we discuss the compatibility of quantum Lefschetz principle and the convergence of quantum cohomology.

There are several cases where the convergence is trivial. If $c_{1}(X)>0$, the small quantum cohomology of $X$ is defined over the polynomial ring by the degree constraints. If $c_{1}(X)<0$, even the big quantum cohomology is defined over the polynomial ring for the same reason. Hence, the problem is the intermediate case, i.e. when there exist two curves $C_{1}, C_{2}$ in $X$ such that $\left\langle c_{1}(X),\left[C_{1}\right]\right\rangle \geq 0$ and $\left\langle c_{1}(X),\left[C_{2}\right]\right\rangle \leq 0$. The main theorem in this paper is the following.

Theorem 1.1. Let $X$ be a smooth projective variety and $\mathcal{L}$ be a nef line bundle on $X$. If the big quantum cohomology $Q H^{*}(X)$ of $X$ has convergent structure constants, then the twisted quantum cohomology $Q H_{S^{1}}^{*}(X, \mathcal{L})$ by $\mathcal{L}$ also has convergent structure constants. (This holds true when $\mathcal{L}$ is replaced by a sum of nef line bundles. )

Coates-Givental's quantum Lefschetz theorem [4] gives the twisted quantum cohomology $Q H_{S^{1}}^{*}(X, \mathcal{L})$ in terms of $Q H^{*}(X)$. Here, $Q H_{S^{1}}^{*}(X, \mathcal{L})$ is a cohomology theory closely related to the quantum cohomology of an intersection $Y \subset X$ with respect to the line bundle $\mathcal{L}$. More precisely, $Q H_{S^{1}}^{*}(X, \mathcal{L})$ gives us the information on the structure constants of $Q H^{*}(Y)$ with respect to the cohomology classes coming from the ambient space $X$. Therefore, if the convergence of $Q H^{*}(X)$ is known, we can know the convergence of $Q H^{*}(Y)$ partially. The main tool in the proof is a ring of formal power series with certain estimates for coefficients.

In the second half of the paper, we give a description of mirror symmetry for a not necessarily nef toric variety. In [17], the author calculated the quantum cohomology $D$-module of a toric variety $X$. The method there was to embed $X$ into another Fano toric variety $X^{\prime}$ as a complete intersection and to use quantum Lefschetz theorem 4] together with a mirror theorem for a Fano toric variety [10. We will recast the consequences of [17] in terms of the following oscillatory integral, which was introduced as a mirror of a toric variety in [8, 10]:

$$
\mathcal{I}_{\Gamma}\left(q_{1}, \ldots, q_{r}, \hbar\right)=\int_{\Gamma_{q} \subset Y_{q}} e^{\sum_{i=1}^{r+N} \mathrm{w}_{i} / \hbar} \omega_{q}, \quad Y_{q}=\left\{\left(\mathrm{w}_{i}\right)_{i=1}^{r+N} \in\left(\mathbb{C}^{*}\right)^{r+N} ; \prod_{i=1}^{r+N} \mathrm{w}_{i}^{m_{i a}}=q_{a}\right\} .
$$


Here, $\omega_{q}$ is a holomorphic volume form on $Y_{q}$ and $\Gamma_{q}$ is a non-compact cycle. These oscillatory integrals define the following mirror $D$-module $M_{\text {mir }}$ (denoted by $F H_{0}$ in the main text):

$$
M_{\text {mir }}:=\mathbb{C}\left\langle q_{1}, \ldots, q_{r}, \hbar \partial_{1}, \ldots, \hbar \partial_{r}, \hbar\right\rangle / I_{\text {poly }}, \quad \partial_{a}=q_{a} \partial / \partial q_{a} .
$$

Here, $I_{\text {poly }}$ is a left ideal consisting of polynomial differential operators annihilating $\mathcal{I}_{\Gamma}(q, \hbar)$. Mirror symmetry for a Fano toric variety $X$ states that the mirror $D$-module $M_{\text {mir }}$ is isomorphic to the big quantum cohomology $D$-module $Q D M^{*}(X)$ restricted to $H^{2}(X)$. Here, $Q D M^{*}(X)$ is a $D$-module over the total cohomology ring $H^{*}(X)$ which is defined by $Q H^{*}(X)$. For a general toric variety $X$, we obtain the following description:

Theorem 1.2 (see Theorem [5.6 for details). Let $\widehat{M}_{\text {mir }}$ be a D-module on the formal germ $\left(\mathbb{C}^{r}, 0\right)$ obtained as the completion of $M_{\text {mir }}$ with respect to its natural q-adic topology (denoted by $F H_{S^{1}}^{*}$ in the main text). There exists a formal embedding $\mathfrak{e m b}:\left(\mathbb{C}^{r}, 0\right) \rightarrow\left(H^{*}(X), 0\right)$ such that we have an isomorphism of D-modules:

$$
\Phi_{\mathfrak{e m b}}: \mathfrak{e m b}^{*}\left(Q D M^{*}(X)\right) \cong \widehat{M}_{\text {mir }} .
$$

Here, $Q D M^{*}(X)$ is the big quantum $D$-module of the toric variety $X$. If $X$ is Fano, the image of $\mathfrak{e m b}$ coincides with the linear subspace $H^{2}(X) \subset H^{*}(X)$.

Because of the completion in the above description, it is not clear if $Q H^{*}(X)$ is convergent. Our main theorem 1.1 is not directly applicable to $X$ because $X$ is a complete intersection in $X^{\prime}$ with respect to a sum of not necessarily nef line bundles. Using techniques similar to the proof of Theorem 1.1, however, we show the following:

Theorem 1.3 (Theorem 5.7 Corollary [5.12). The big quantum cohomology of a smooth projective toric variety is convergent and generically semisimple. The embedding $\mathfrak{e m b}$ in the above theorem is complex analytic.

Note that the isomorphism $\Phi_{\mathfrak{e m b}}$ is not convergent unless $X$ is nef (Proposition 5.13). The asymptotic expansion of the oscillatory integral $\mathcal{I}_{\Gamma}(q, \hbar)$ in $\hbar$ is shown to give a formal solution to $Q D M^{*}(X)$ for special choices of cycles $\Gamma$ (Corollary 6.9). We also prove the $R$-conjecture for equivariant quantum cohomology of toric varieties (Theorem 6.10). Here, the $R$-conjecture implies the Virasoro constraints by Givental's theory [13.

Our result on the semisimplicity is also a successful test for Bayer and Manin's modified Dubrovin's conjecture [1. The modified Dubrovin's conjecture claims that $(p, p)$-part of quantum cohomology of a projective variety $X$ is generically semisimple if its bounded derived category $D_{\text {coh }}^{b}(X)$ of coherent sheaves admits a full exceptional collection. In fact, Kawamata recently showed that toric varieties have full exceptional collections [21].

For the application of the main theorem 1.1, we need to know the convergence of big quantum cohomology of ambient spaces. We prove that if $H^{*}(X)$ is generated by $H^{2}(X)$ and if the small quantum cohomology of $X$ has convergent structure constants, so does the big quantum cohomology (Corollary [5.9). In particular, the big quantum cohomology of a Fano variety with $H^{2}$-generated cohomology always has convergent structure constants. In a subsequent paper [18, we will also prove that the big quantum cohomology (and higher genus potential $\mathcal{F}_{g}$ also) is convergent for a projective manifold which admits Hamiltonian torus action with only isolated fixed points and isolated one dimensional orbits.

We should remark that in this paper, we only consider the even part of (quantum) cohomology. For example, $H^{*}(X)$ always means $H^{\text {even }}(X)$.

The paper is organized as follows. In section 2 and 3 , we review the quantum $D$-modules and the quantum Lefschetz theorem by Coates and Givental. In section 4 , we prove the main 
theorem. In section 5, we discuss the mirror symmetry for a non-nef toric variety. In section 6 , we prove the $R$-conjecture for any toric variety.

Acknowledgments. Thanks are due to Professor Martin Guest, Professor Hiraku Nakajima and Kazushi Ueda for valuable discussions. Part of this paper was written while the author stayed at Mathematical Sciences Research Institute. He thanks MSRI for excellent working conditions. He is also grateful to anonymous referee for thier helpful comments. This research is supported by Grant-in-Aid for JSPS Fellows and Scientific Research 15-5482.

\section{Quantum $D$-Modules}

In this section, we introduce quantum $D$-modules twisted by the equivariant Euler class following [4, 25]. Let $X$ be a smooth projective variety and $\mathcal{L}$ be a line bundle over $X$. Let $\bar{M}_{0, n}(X, \boldsymbol{d})$ be the moduli space of genus 0 , degree $\boldsymbol{d}$ stable maps to $X$ with $n$ marked points, where $\boldsymbol{d} \in H_{2}(X, \mathbb{Z})$. We have the following diagram:

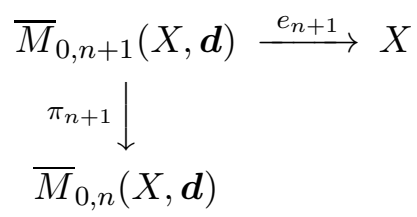

where $e_{i}$ is the evaluation map and $\pi_{i}$ is the forgetful map. We introduce the fiber-wise $S^{1}$ action on $\mathcal{L}$ by scalar multiplication. Let $\lambda$ be a generator of the $S^{1}$ equivariant cohomology of a point. Define the twisted correlator

$$
\left\langle\alpha_{1}, \ldots, \alpha_{n}\right\rangle_{S^{1}, \boldsymbol{d}}^{\mathcal{L}}=\int_{\left[\bar{M}_{0, n}(X, \boldsymbol{d})\right]^{\mathrm{virt}}} \prod_{i=1}^{n} e_{i}^{*}\left(\alpha_{i}\right) \operatorname{Euler}_{S^{1}}\left(R^{\bullet} \pi_{n+1_{*}} e_{n+1}^{*} \mathcal{L}\right),
$$

where $\alpha_{1}, \ldots, \alpha_{n} \in H^{*}(X, \mathbb{C})$ and $\left[\bar{M}_{0, n}(X, \boldsymbol{d})\right]^{\text {virt }}$ is the virtual fundamental class. The right hand side is in $\mathbb{C}\left[\lambda, \lambda^{-1}\right]$. Let $\left\{p_{0}, \ldots, p_{s}\right\}$ be a basis of $H^{*}(X, \mathbb{C})$. We assume that $p_{0}$ is a unit and that $p_{1}, \ldots, p_{r}$ form a nef integral basis of $H^{2}(X, \mathbb{Z})(r \leq s)$. Let $t_{0}, \ldots, t_{s}$ be linear coordinates dual to the basis $p_{0}, \ldots, p_{s}$. We write $q_{a}:=\exp \left(t_{a}\right)$ and $q^{\boldsymbol{d}}:=q_{1}^{\left\langle p_{1}, \boldsymbol{d}\right\rangle} q_{2}^{\left\langle p_{2}, \boldsymbol{d}\right\rangle} \cdots q_{r}^{\left\langle p_{r}, \boldsymbol{d}\right\rangle}=$ $\exp \left(\sum_{a=1}^{r}\left\langle p_{a}, \boldsymbol{d}\right\rangle t_{a}\right)$ for $\boldsymbol{d} \in H_{2}(X, \mathbb{Z})$. Define a twisted pairing $\langle\cdot, \cdot\rangle_{S^{1}}^{\mathcal{L}}$ by

$$
\langle\alpha, \beta\rangle_{S^{1}}^{\mathcal{L}}=\int_{X} \alpha \cup \beta \cup \operatorname{Euler}_{S^{1}}(\mathcal{L}) .
$$

The twisted quantum product $*_{\mathcal{L}}$ is defined by the formula:

$$
\begin{aligned}
\left\langle\alpha *_{\mathcal{L}} \beta, \gamma\right\rangle_{S^{1}}^{\mathcal{L}} & =\sum_{\boldsymbol{d} \in \Lambda} \sum_{n \geq 0} \frac{1}{n !}\left\langle\alpha, \beta, \gamma,\left(\sum_{j=0}^{s} t_{j} p_{j}\right)^{\otimes n}\right\rangle_{S^{1}, \boldsymbol{d}}^{\mathcal{L}} \\
& =\sum_{\boldsymbol{d} \in \Lambda} \sum_{n \geq 0} \frac{1}{n !}\left\langle\alpha, \beta, \gamma,\left(\sum_{j=r+1}^{s} t_{j} p_{j}\right)^{\otimes n}\right\rangle_{S^{1}, \boldsymbol{d}}^{\mathcal{L}} q^{\boldsymbol{d}},
\end{aligned}
$$

where $\alpha, \beta, \gamma \in H^{*}(X, \mathbb{C})$ and $\Lambda \subset H_{2}(X, \mathbb{Z})$ is a semigroup generated by effective curves. The product $*_{\mathcal{L}}$ is extended linearly over $\mathbb{C}\left[\lambda, \lambda^{-1}\right] \llbracket t_{0}, q_{1}, \ldots, q_{r}, t_{r+1}, \ldots, t_{s} \rrbracket$ and

$$
Q H_{S^{1}}^{*}(X, \mathcal{L}):=\left(H^{*}(X) \otimes \mathbb{C}\left[\lambda, \lambda^{-1}\right] \llbracket t_{0}, q_{1}, \ldots, q_{r}, t_{r+1}, \ldots, t_{s} \rrbracket, *_{\mathcal{L}}\right)
$$

becomes an associative and commutative ring. We define $\operatorname{deg} t_{j}=2-\operatorname{deg} p_{j}$ for $j=0$ or $j>r$, $\operatorname{deg} q^{\boldsymbol{d}}=2\left\langle c_{1}(X)-c_{1}(\mathcal{L}), \boldsymbol{d}\right\rangle$ and $\operatorname{deg} \lambda=2$. Then $Q H_{S^{1}}^{*}(X, \mathcal{L})$ becomes a graded ring. For 
simplicity, we also use the following notation:

$$
x=\left(x_{0}, x_{1}, \ldots, x_{r}, x_{r+1}, \ldots, x_{s}\right)=\left(t_{0}, q_{1}, \ldots, q_{r}, t_{r+1}, \ldots, t_{s}\right), \quad \tau=\sum_{j=0}^{s} t_{j} p_{j} .
$$

When $c_{1}(\mathcal{L})$ is nef, the product $*_{\mathcal{L}}$ can be defined over $\mathbb{C}[\lambda] \llbracket x \rrbracket$ (see $[25]$ ). Therefore in this case, we can also consider the non-equivariant $(\lambda=0)$ version $Q H^{*}(X, \mathcal{L})=\left(H^{*}(X) \otimes \mathbb{C} \llbracket x \rrbracket, *_{\mathcal{L}}\right)$.

The usual (non-twisted) quantum cohomology $Q H^{*}(X)=\left(H^{*}(X) \otimes \mathbb{C} \llbracket x \rrbracket, *_{X}\right)$ is defined by removing all the Euler classes in the definition. Let $Y$ be a smooth intersection in $X$ with respect to a transverse section $s \in \Gamma(X, \mathcal{L})$. By the main theorem of [22], if $c_{1}(\mathcal{L})$ is nef, we have at $\lambda=0$,

$$
\left\langle\alpha_{1}, \ldots, \alpha_{n}\right\rangle_{\boldsymbol{d}}^{\mathcal{L}}=\left\langle i^{*} \alpha_{1}, \ldots, i^{*} \alpha_{n}\right\rangle_{\boldsymbol{d}}^{Y}
$$

for the inclusion $i: Y \rightarrow X$ and the correlator $\langle\cdots\rangle_{\boldsymbol{d}}^{Y}$ for $Y$. Therefore, from $*_{\mathcal{L}}$, we can read the structure constants of $Q H^{*}(Y)$ with respect to the classes coming from the ambient space.

We can endow a $D$-module structure on the quantum cohomology. Let $*$ denote $*_{\mathcal{L}}$ or $*_{X}$. The dual Givental connection $\nabla^{\hbar}$ is defined by

$$
\nabla_{j}^{\hbar}=\hbar \frac{\partial}{\partial t_{j}}+p_{j} * \quad(0 \leq j \leq s),
$$

where $\hbar$ is a formal variable of degree two. This connection is regular singular along $q_{1}=\cdots=$ $q_{r}=0$ and is known to be flat. It defines the non-twisted or twisted quantum $D$-modules:

$$
Q D M^{*}(X)=\left(H^{*}(X)[\hbar] \llbracket x \rrbracket, \nabla^{\hbar}\right), \quad Q D M_{S^{1}}^{*}(X, \mathcal{L})=\left(H^{*}(X)\left[\hbar, \lambda, \lambda^{-1}\right] \llbracket x \rrbracket, \nabla^{\hbar}\right)
$$

When $c_{1}(L)$ is nef, we can also consider the non-equivariant version $Q D M^{*}(X, \mathcal{L})$. It is easy to see that quantum $D$-module is generated by 1 over the Heisenberg algebra $\mathbb{C}[\hbar] \llbracket x \rrbracket\left[\nabla_{0}^{\hbar}, \ldots, \nabla_{s}^{\hbar}\right]$. There exists a unique fundamental solution $L(\tau, \hbar)$ for the flat connection $\nabla^{\hbar}$ such that

$$
\begin{aligned}
& \hbar d \circ L(\tau, \hbar)=L(\tau, \hbar) \circ \nabla^{\hbar}, \\
& L(\tau, \hbar)=e^{\tau / \hbar} T(x, \hbar), \quad T \in \operatorname{End}\left(H^{*}(X)\right) \otimes \mathbb{C}\left[\hbar^{-1}\right] \llbracket x \rrbracket,\left.\quad T\right|_{q=0}=\mathrm{id} .
\end{aligned}
$$

where $\tau \in H^{*}(X)$ is considered as an operator acting on $H^{*}(X)$ by the cup product. This $L(\tau, \hbar)$ satisfies the following unitarity [11]:

$$
\langle L(\tau,-\hbar) \alpha, L(\tau, \hbar) \beta\rangle=\langle\alpha, \beta\rangle
$$

where $\langle\cdot, \cdot\rangle$ denotes the Poincaré pairing $\langle\cdot, \cdot\rangle^{X}$ of $X$ in case of $Q D M^{*}(X)$ and the twisted Poincaré pairing $\langle\cdot, \cdot\rangle_{S^{1}}^{\mathcal{L}}$ in case of $Q D M_{S^{1}}^{*}(X, \mathcal{L})$. This $L(\tau, \hbar)$ also defines the $J$-function of quantum cohomology by

$$
J(\tau, \hbar):=L(\tau, \hbar) 1, \quad 1 \in H^{*}(X) \text { is a unit. }
$$

The $J$-function is a realization of a generator 1 of the quantum $D$-module as a function.

\section{Coates-Givental's Quantum Lefschetz}

Coates and Givental's quantum Lefschetz theorem [4] describes the relationship between the two quantum cohomologies $Q H^{*}(X)$ and $Q H_{S^{1}}^{*}(X, \mathcal{L})$. It was described in terms of symplectic transformations of Lagrangian cones in the infinite dimensional space $H^{*}(X) \otimes \mathbb{C} \llbracket \hbar, \hbar^{-1} \rrbracket$. In this paper, we describe it in terms of gauge transformations by translating the language of cones into that of quantum $D$-modules. 
3.1. Symplectic formalism. First we will review the infinite dimensional symplectic formalism in [13, 4] briefly. Consider the following general multiplicative characteristic class for a complex vector bundle $E$ :

$$
\boldsymbol{c}(E)=\exp \left(\sum_{k=0}^{\infty} s_{k} \operatorname{ch}_{k}(E)\right) .
$$

Here, $s=\left(s_{0}, s_{1}, s_{2}, \ldots\right)$ are infinite number of arbitrary parameters. Let $\mathcal{H}$ be the following infinite dimensional vector space:

$$
\mathcal{H}:=H^{*}(X) \otimes \mathbb{C}\left[\hbar, \hbar^{-1}\right] \llbracket s \rrbracket=\mathcal{H}_{+} \oplus \mathcal{H}_{-},
$$

where

$$
\mathcal{H}_{+}=H^{*}(X) \otimes \mathbb{C}[\hbar] \llbracket s \rrbracket, \quad \mathcal{H}_{-}=\hbar^{-1} H^{*}(X) \otimes \mathbb{C}\left[\hbar^{-1}\right] \llbracket s \rrbracket .
$$

For any holomorphic vector bundle $E$ on $X$, define a symplectic form $\Omega_{\boldsymbol{s}}$ on $\mathcal{H}$ by

$$
\Omega_{\boldsymbol{s}}(f(\hbar), g(\hbar))=\operatorname{Res}_{\hbar=0} d \hbar \int_{X} f(-\hbar) g(\hbar) \boldsymbol{c}(E), \quad f, g \in \mathcal{H} .
$$

Then, $\mathcal{H}_{+}$and $\mathcal{H}_{-}$become Lagrangian with respect to this symplectic form $\Omega_{\boldsymbol{s}}$ and give a polarization of $\mathcal{H}$. The fundamental solution $L(\tau, \hbar)$ of the quantum $D$-module $Q D M^{*}(X)$ (see (19)) defines a Lagrangian subspace $\mathbb{L}_{\tau}$ of $\left(\mathcal{H}, \Omega_{0}\right)$ for each $\tau$.

$$
\mathbb{L}_{\tau}:=L(\tau,-\hbar)\left(\mathcal{H}_{+}\right)
$$

Here, we assume the convergence of $L(\tau, \hbar)$ for the sake of simplicity. For a rigorous argument, we need to introduce a Novikov ring $\Lambda_{\text {nov }}$ and replace $\mathcal{H}$ with the module $H^{*}\left(X, \Lambda_{\text {nov }} \llbracket s \rrbracket\right)\left\{\hbar, \hbar^{-1}\right\}$ of convergent power series in $\hbar$ with respect to the adic topology (as explained in [4]). These semi-infinite subspaces sweep a germ of Lagrangian cone $\mathcal{C}_{0}$ in $\left(\mathcal{H}, \Omega_{0}\right)$.

$$
\mathcal{C}_{0}:=\bigcup_{\tau \in H^{*}(X)} \hbar \mathbb{L}_{\tau}
$$

As explained in 4], the tangent space of $\mathcal{C}_{0}$ at any point in $\hbar \mathbb{L}_{\tau}$ equals the Lagrangian subspace $\mathbb{L}_{\tau}$. In other words, $\mathcal{C}_{0}$ has a remarkable property that it is ruled by $\hbar$ times its tangent spaces. Let $J(\tau, \hbar)=L(\tau, \hbar) 1$ be the $J$-function of $Q H^{*}(X)$. Then the vectors $-\hbar J(\tau,-\hbar)$ parametrized by $\tau \in H^{*}(X)$ lie on the cone $\mathcal{C}_{0}$. The derivatives $\left\{-\hbar \partial_{t_{j}} J(\tau,-\hbar)\right\}_{j}$ form a basis of the tangent space $\mathbb{L}_{\tau}$ of $\mathcal{C}_{0}$ over $\mathbb{C}[\hbar] \llbracket s \rrbracket$. Thus, the $J$-function recovers the whole Lagrangian cone $\mathcal{C}_{0}$ by (4).

Similarly, the twisted theory by the characteristic class $c$ and a vector bundle $E$ defines a Lagrangian cone $\mathcal{C}_{\boldsymbol{s}} \subset\left(\mathcal{H}, \Omega_{\boldsymbol{s}}\right)$. Coates and Givental proved that two cones $\mathcal{C}_{0}$ and $\mathcal{C}_{\boldsymbol{s}}$ are related by a linear symplectic transformation.

Theorem 3.1 (4, Corollary 4). The linear symplectic transformation

$$
\boldsymbol{c}(E)^{-1 / 2} \exp \left(\sum_{l, k \geq 0} s_{2 k+l-1} \frac{B_{2 k}}{(2 k) !} \operatorname{ch}_{l}(E) \hbar^{2 k-1}\right):\left(\mathcal{H}, \Omega_{0}\right) \longrightarrow\left(\mathcal{H}, \Omega_{\boldsymbol{s}}\right)
$$

sends the Lagrangian cone $\mathcal{C}_{0}$ to $\mathcal{C}_{\boldsymbol{s}}$. Here, $B_{2 k}$ is the Bernoulli number defined by $x /\left(1-e^{-x}\right)=$ $x / 2+\sum_{k \geq 0} B_{2 k} x^{2 k} /(2 k)$ ! and $s_{-1}=0$.

In this paper, we only consider a twist by the equivariant Euler class and the case where $E$ is a line bundle $\mathcal{L}$. In this case, values of the parameters $s_{i}$ are set as follows:

$$
s_{0}=\log \lambda, \quad s_{k}=(-1)^{k-1} \frac{(k-1) !}{\lambda^{k}} \quad k>0 .
$$


Using this substitution, $\boldsymbol{c}(\mathcal{L})$ equals the equivariant Euler class $c_{1}(\mathcal{L})+\lambda$, where $S^{1}$ acts on $\mathcal{L}$ by scalar multiplication on each fiber and $\lambda$ is a generator of $H_{S^{1}}^{*}(\mathrm{pt})$. Coates and Givental introduced the following hypergeometric modification of the $J$-function:

$$
I_{\mathcal{L}}(\tau, \hbar):=\sum_{\boldsymbol{d} \in \Lambda} \frac{\prod_{k=-\infty}^{\langle\rho, \boldsymbol{d}\rangle}(\rho+k \hbar+\lambda)}{\prod_{k=-\infty}^{0}(\rho+k \hbar+\lambda)} J_{\boldsymbol{d}}(t, \hbar) q^{\boldsymbol{d}}, \quad \rho:=c_{1}(\mathcal{L}),
$$

where $J(\tau, \hbar)=\sum_{\boldsymbol{d} \in \Lambda} J_{\boldsymbol{d}}(t, \hbar) q^{\boldsymbol{d}}$ is the $J$-function of $Q H^{*}(X)$.

Theorem 3.2 ([4, Theorem 2]). The vectors $-\hbar I_{\mathcal{L}}\left(\tau^{*},-\hbar\right)$ parametrized by $\tau^{*} \in H^{*}(X)$ lie on the cone $\mathcal{C}_{\lambda}$ defined by the twisted quantum cohomology $Q H_{S^{1}}^{*}(X, \mathcal{L})$. The derivatives $\left\{-\hbar \partial_{t_{j}} I_{\mathcal{L}}\left(\tau^{*},-\hbar\right)\right\}_{j}$ span the tangent spaces to $\mathcal{C}_{\lambda}$ and reconstruct the whole cone $\mathcal{C}_{\lambda}$.

3.2. Symplectic transformation as a gauge transformation. Following [17, section 5], we will interpret the linear symplectic transformation in Theorem 3.1 as a gauge transformation of quantum $D$-modules. The quantum $D$-module $Q D M^{*}(X)=\left(H^{*}(X) \otimes \mathbb{C}[\hbar] \llbracket x \rrbracket, \nabla^{\hbar}\right)$ is a vector bundle on a formal neighborhood Spec $\mathbb{C} \llbracket x_{0}, \ldots, x_{s} \rrbracket$ with fiber $H^{*}(X)$ endowed with a flat connection $\nabla^{\hbar}$ with parameter $\hbar$. This vector bundle has a canonical trivialization by definition. This canonical trivialization together with a canonical origin $\tau=0$ of the cohomology fixes a choice of a fundamental solution (10) and in turn defines the Lagrangian subspace (3) and the Lagrangian cone (4). Suppose that one changes the trivialization by a gauge transformation $g(x, \hbar) \in \operatorname{End}\left(H^{*}(X)\right) \otimes \mathbb{C}[\hbar] \llbracket x \rrbracket$ such that

$$
g_{0}(\hbar):=\left.g\right|_{q=0}=A_{0} \exp \left(a_{1} \hbar+a_{3} \hbar^{3}+\cdots\right), \quad A_{0}, a_{i} \in H^{*}(X) .
$$

Then the dual Givental connection $\nabla^{\hbar}$ changes as

$$
g^{*} \nabla_{j}^{\hbar}=g^{-1} \circ \nabla_{j}^{\hbar} \circ g=\hbar \frac{\partial}{\partial t_{j}}+g^{-1}\left(p_{j} *\right) g+\hbar g^{-1} \frac{\partial g}{\partial t_{j}}
$$

Suppose also that one takes another point $\tau=c$ as an origin. Then the fundamental solution changes as

$$
L^{g, c}(\tau, \hbar)=e^{-c / \hbar} g_{0}(\hbar)^{-1} L(\tau, \hbar) g(x, \hbar) .
$$

This new fundamental solution $L^{g, c}(\tau, \hbar)$ is uniquely determined by a differential equation $\hbar d \circ L^{g, c}(\tau, \hbar)=L^{g, c}(\tau, \hbar) \circ\left(g^{*} \nabla^{\hbar}\right)$ and a shifted initial condition:

$$
L^{g, c}(\tau, \hbar)=e^{(\tau-c) / \hbar} T^{g, c}(x, \hbar),\left.\quad T^{g, c}\right|_{q=0}=\mathrm{id} .
$$

By the same formulas (3), (44) as before, $L^{g, c}(\tau, \hbar)$ defines the following Lagrangian cone:

$$
e^{c / \hbar} g_{0}(-\hbar)^{-1} \mathcal{C}_{0}=A_{0}^{-1} \exp \left(\frac{c}{\hbar}+a_{1} \hbar+a_{3} \hbar^{3}+\cdots\right) \mathcal{C}_{0} .
$$

Therefore, $e^{c / \hbar} g_{0}(-\hbar)^{-1}$ is identified with the symplectic transformation in Theorem 3.1. In case of equivariant Euler class, Theorem 3.1 is restated as follows:

Proposition 3.3. The dual Givental connection of the twisted quantum $D$-module $Q D M_{S^{1}}^{*}(X, \mathcal{L})$ is obtained from that of $Q D M^{*}(X)$ by a gauge transformation $\nabla^{\hbar} \mapsto g^{*} \nabla^{\hbar}=g^{-1} \circ \nabla^{\hbar} \circ g$, $g(x, \hbar) \in \sqrt{\lambda} \operatorname{End}\left(H^{*}(X)\right) \otimes \mathbb{C}[\hbar]\left[\lambda, \lambda^{-1} \rrbracket \llbracket x \rrbracket\right.$ and a coordinate change $\tau \mapsto \hat{\tau}=\hat{\tau}(\tau)$ such that

$$
\begin{gathered}
\left.g\right|_{q=0}=(\lambda+\rho)^{1 / 2} \exp \left(\sum_{k \geq 1, l \geq 0}(-1)^{l} \frac{(2 k+l-2) !}{\lambda^{2 k+l-1}} \frac{B_{2 k}}{(2 k) !} \frac{\rho^{l}}{l !} \hbar^{2 k-1}\right) . \\
\hat{\tau}(\tau=c)=0, \quad \text { where } c=\rho \log \lambda+\sum_{l=2}^{\operatorname{dim} X}(-1)^{l} \frac{(l-2) !}{\lambda^{l-1}} \frac{\rho^{l}}{l !} .
\end{gathered}
$$


The hypergeometric modification in Theorem 3.2 can be considered as an intermediate step to find a gauge transformation $g$ and a coordinate change $\hat{\tau}=\hat{\tau}(\tau)$ in the above proposition. If one changes a trivialization of $Q D M^{*}(X)$ by a gauge transformation $g$ satisfying (77) and also shifts the origin by a coordinate change satisfying (8), the set of column vectors $L^{g, c}(\tau,-\hbar) p_{j}$ of the new fundamental solution gives a basis of tangent spaces to the cone $\mathcal{C}_{\lambda}$ defined by the twisted quantum cohomology. Conversely, a matrix formed by the basis $\left\{-\hbar \partial_{t_{j}} I_{\mathcal{L}}\left(\tau^{*},-\hbar\right)\right\}_{j}$ of tangent spaces to the cone $\mathcal{C}_{\lambda}$ (given in Theorem 3.2) satisfies the following initial condition:

$$
\widetilde{L}\left(\tau^{*}, \hbar\right):=\left[\begin{array}{ccc}
\mid & & \mid \\
\hbar \partial_{t_{0}} I_{\mathcal{L}}\left(\tau^{*}, \hbar\right) & \cdots & \hbar \partial_{t_{s}} I_{\mathcal{L}}\left(\tau^{*}, \hbar\right) \\
\mid & & \mid
\end{array}\right]=e^{\tau^{*} / \hbar} \widetilde{T}(x, \hbar),\left.\quad \widetilde{T}(x, \hbar)\right|_{q=0}=\mathrm{id} .
$$

From this it follows that there exists an intermediate gauge transformation $g_{1}(\tau, \hbar)$ satisfying (17) and a coordinate change $\tau^{*}=\tau^{*}(\tau)$ satisfying (8) such that

$$
L^{g_{1}, c}(\tau, \hbar)=\widetilde{L}\left(\tau^{*}, \hbar\right),
$$

where the left hand side is given in (6). Let $\widetilde{\nabla}^{\hbar}$ be the connection in this new gauge and coordinates:

$$
\widetilde{\nabla}^{\hbar}:=\hbar d+\sum_{j=0}^{s} \Omega_{j} d t_{j}, \quad \Omega_{j}(x, \hbar):=\widetilde{L}(\tau, \hbar)^{-1} \hbar \partial_{t_{j}} \widetilde{L}(\tau, \hbar) .
$$

Here, the connection $\widetilde{\nabla}^{\hbar}$ at a point $\tau^{*}(\tau)$ is equal to $g_{1}^{*} \nabla^{\hbar}$ at $\tau$. By abuse of notation, we use $\tau$ instead of $\tau^{*}$ as an argument of this new connection $\widetilde{\nabla}^{\hbar}$ and solution $\widetilde{L}$.

Proposition 3.4. (i) The connection matrix $\Omega_{j}$ is in the ring $\operatorname{End}\left(H^{*}(X)\right) \otimes \mathbb{C}[\hbar]\left[\lambda, \lambda^{-1} \rrbracket \llbracket x \rrbracket\right.$. If $c_{1}(\mathcal{L})$ is nef, this is also in the ring $\operatorname{End}\left(H^{*}(X)\right) \otimes \mathbb{C}[\hbar, \lambda] \llbracket x \rrbracket$.

(ii) The pairing $\langle\langle\alpha, \beta\rangle\rangle$ defined by

$$
\langle\langle\alpha, \beta\rangle\rangle:=\langle\widetilde{L}(\tau,-\hbar) \alpha, \widetilde{L}(\tau, \hbar) \beta\rangle_{S^{1}}^{\mathcal{L}}, \quad \alpha, \beta \in H^{*}(X)
$$

takes values in $\mathbb{C}[\hbar]\left[\lambda, \lambda^{-1} \rrbracket \llbracket x \rrbracket\right.$. If $c_{1}(\mathcal{L})$ is nef, this takes values in $\mathbb{C}[\hbar, \lambda] \llbracket x \rrbracket$.

Proof. (i) A tangent space to the cone $\mathcal{C}_{\lambda}$ is a vector space over $\mathbb{C}[\hbar]\left[\lambda, \lambda^{-1} \rrbracket\right.$. The former part follows from this and the argument in [4] using a ruling property (4) of the cone $\mathcal{C}_{\lambda}$. If $c_{1}(\mathcal{L})$ is nef, the hypergeometric modification is of the form

$$
I_{\mathcal{L}}(\tau, \hbar)=\sum_{\boldsymbol{d} \in \Lambda} \prod_{k=1}^{\langle\rho, \boldsymbol{d}\rangle}(\rho+\lambda+k \hbar) J_{\boldsymbol{d}}(t, \hbar) q^{\boldsymbol{d}}
$$

and does not contain negative powers of $\lambda$. The latter part follows from this.

(ii) This follows from that $\widetilde{L}(\tau,-\hbar) \alpha$ is a tangent vector of $\mathcal{C}_{\lambda}$ and that $\mathcal{C}_{\lambda}$ is Lagrangian with respect to the symplectic form $\operatorname{Res}_{\hbar=0}\langle f(-\hbar), g(\hbar)\rangle_{S^{1}}^{\mathcal{L}} d \hbar$.

The dual Givental connection of $Q D M_{S^{1}}^{*}(X, \mathcal{L})$ can be obtained from $\widetilde{\nabla}^{\hbar}$ by a further gauge transformation by $g_{2} \in \operatorname{End}\left(H^{*}(X)\right) \otimes \mathbb{C}[\hbar]\left[\lambda, \lambda^{-1} \rrbracket \llbracket x \rrbracket\right.$ and a coordinate change $x \mapsto \hat{x}$. The gauge transformation $g_{2}$ must satisfy

$$
\left.g_{2}\right|_{q=0}=\mathrm{id}, \quad g_{2}^{*} \widetilde{\nabla}^{\hbar}=\hbar d+\sum_{j=0}^{s} \widehat{\Omega}_{j} d t_{j}, \quad \widehat{\Omega}_{j} \text { does not depend on } \hbar .
$$


The new coordinates $\hat{x}=\left(\hat{t}_{0}, \hat{q}_{1}=e^{\hat{t}_{1}}, \ldots, \hat{q}_{r}=e^{\hat{t}_{r}}, \hat{t}_{r+1}, \ldots, \hat{t}_{s}\right)$ are of the form

$$
\begin{gathered}
\hat{t}_{0}=t_{0}+F_{0}(x, \lambda), \quad \log \hat{q}_{a}=\log q_{a}+F_{a}(x, \lambda) \quad(1 \leq a \leq r), \\
\hat{t}_{j}=t_{j}+F_{j}(x, \lambda), \quad(r+1 \leq j \leq s)
\end{gathered}
$$

for some $F_{j}(x, \lambda) \in \mathbb{C}\left[\lambda, \lambda^{-1} \rrbracket \llbracket x \rrbracket\right.$ satisfying $\left.F_{j}(x, \lambda)\right|_{q=0}=0$. When written in the new coordinate system $\left(\hat{t}_{0}, \ldots, \hat{t}_{s}\right)$, the connection matrix $\widehat{\Omega}_{\hat{j}}$ must satisfy

$$
\widehat{\Omega}_{\hat{j}}(1)=p_{j}, \quad \widehat{\Omega}_{\hat{j}}:=\sum_{i=0}^{s} \frac{\partial t_{i}}{\partial \hat{t}_{j}} \widehat{\Omega}_{i}
$$

since $\widehat{\Omega}_{\hat{j}}$ is identified with the quantum multiplication by $p_{j}$ in $Q H_{S^{1}}^{*}(X, \mathcal{L})$. Note that a gauge transformation and a coordinate change satisfying (11), (12) do not change the cone $\mathcal{C}_{\lambda}$. As shown in [4, 14, this gauge transformation $g_{2}$ can be obtained as the positive part of the Birkhoff factorization of the fundamental solution:

$$
g_{2}=\widetilde{L}_{+}, \quad \widetilde{L}(\tau, \hbar)=\widetilde{L}_{-}(\tau, \hbar) \widetilde{L}_{+}(x, \hbar),
$$

where $\widetilde{L}_{-}=\mathrm{id}+O\left(\hbar^{-1}\right)$ and $\widetilde{L}_{+}$is regular at $\hbar=0$. As shown in [17, Theorem 4.6 and 4.8, $g_{2}$ and $\hat{x}$ can be uniquely determined by the conditions (11), (13).

Summarizing, we can find the gauge transformation $g$ in Proposition 3.3 as a composite $g_{1} \circ g_{2}$ of two gauge transformations. First gauge transformation $g_{1}$ corresponds to the hypergeometric modification $\widetilde{L}(\tau, \hbar)$ of the fundamental solution and the second one $g_{2}$ can be obtained by the Birkhoff factorization of $\widetilde{L}(\tau, \hbar)$. In the next section, we study the analytic property of the connection $\Omega_{j}$ and the second gauge transformation $g_{2}$ in detail.

\section{Proof of the Main theorem}

4.1. Formal power series with estimates. In this subsection, we give an estimate for the connection matrix $\Omega_{j}$ which is obtained after the gauge transformation $g_{1}$. We will show that matrix elements of $\Omega_{j}$ are not necessarily convergent power series, but their coefficients satisfy certain estimates. We assume that $c_{1}(\mathcal{L})=\rho$ is nef and that the ambient quantum cohomology $Q H^{*}(X)$ converges. We use the notation in Section 3.

Let $L(\tau, \hbar)$ be the fundamental solution (10) of $Q D M^{*}(X)$.

$$
L(\tau, \hbar)=e^{\tau / \hbar} T(x, \hbar)=e^{\tau / \hbar} \sum_{\boldsymbol{m}, n \geq 0} T_{\boldsymbol{m}, n} x^{\boldsymbol{m}} \hbar^{-n},
$$

where $\boldsymbol{m}=\left(m_{1}, \ldots, m_{s}\right)$ is a multi-index in $\mathbb{Z}_{\geq 0}^{s}$. $\left(T(x, \hbar)\right.$ does not depend on $x_{0}=t_{0}$. $)$

Lemma 4.1. There exist positive constants $C_{1}, C_{2}>1$ such that

$$
\left\|T_{\boldsymbol{m}, n}\right\| \leq C_{1} C_{2}^{|\boldsymbol{m}|+n} \frac{1}{n !}
$$

where $\|\cdot\|$ is the operator norm and $|\boldsymbol{m}|=\sum_{j=1}^{s} m_{j}$.

Proof. The function $T(x, \hbar)$ is known to satisfy the following homogeneity:

$$
2 \hbar \frac{\partial T}{\partial \hbar}+\sum_{a=1}^{r}\left(\operatorname{deg} q_{a}\right) \frac{\partial T}{\partial q_{a}}+\sum_{j=r+1}^{s}\left(\operatorname{deg} t_{j}\right) t_{j} \frac{\partial T}{\partial t_{j}}+[\mu, T]=0,
$$


where $\mu$ is a constant matrix defined by $\mu\left(p_{j}\right)=\left(\operatorname{deg} p_{j}\right) p_{j}$ and $\operatorname{deg} q_{a}$ is the degree defined in non-twisted theory. By using $\hbar \partial L / \partial t_{j}=L\left(p_{j} *_{X}\right)$, we can rewrite the above equation as

$$
2 \hbar \frac{\partial T}{\partial \hbar}+\sum_{a=1}^{r}\left(\operatorname{deg} q_{a}\right) \frac{1}{\hbar}\left(T\left(p_{a} *_{X}\right)-p_{a} T\right)+\sum_{j=r+1}^{s}\left(\operatorname{deg} t_{j}\right) \frac{t_{j}}{\hbar} T\left(p_{j} *_{X}\right)+[\mu, T]=0
$$

Set $T_{n}(x)=\sum_{\boldsymbol{m}} T_{\boldsymbol{m}, n} x^{\boldsymbol{m}}$. By this equation, we have

$$
(2 n-\operatorname{ad}(\mu)) T_{n}(x)=T_{n-1}(x) A(x)-c_{1}(X) T_{n-1}(x),
$$

where $A(x)=\sum_{a=1}^{r}\left(\operatorname{deg} q_{a}\right)\left(p_{a} *_{X}\right)+\sum_{j=r+1}^{s} t_{j}\left(\operatorname{deg} t_{j}\right)\left(p_{j} *_{X}\right)$. Therefore, we have

$$
T_{n}=\frac{1}{2 n}\left(1-\frac{\operatorname{ad}(\mu)}{2 n}\right)^{-1}\left(T_{n-1} A-c_{1}(X) T_{n-1}\right)
$$

By the assumption that $Q H^{*}(X)$ is convergent, there exist a neighborhood $U$ of $x=0$ and a constant $C>0$ such that

$$
\sup _{x \in U}\|A(x)\| \leq C, \quad \sup _{n \geq 1}\left\|\left(1-\frac{\operatorname{ad}(\mu)}{2 n}\right)^{-1}\right\| \leq C, \quad\left\|c_{1}(X)\right\| \leq C .
$$

Since $T_{0}(x)=\mathrm{id}$, we can see that $\sup _{x \in U}\left\|T_{n}(x)\right\| \leq C^{2 n} / n$ !. The lemma follows from this estimate.

For a multi-index $\boldsymbol{m}=\left(m_{1}, \ldots, m_{s}\right)$, we write $\langle\rho, \boldsymbol{m}\rangle=\sum_{a=1}^{r} \xi_{a} m_{a}$, where $\rho=c_{1}(\mathcal{L})=$ $\sum_{a=1}^{r} \xi_{a} p_{a}$. Note that $\langle\rho, \boldsymbol{m}\rangle \geq 0$ for $\boldsymbol{m} \in \mathbb{Z}_{\geq 0}^{s}$ because $\rho$ is nef. Let $\widetilde{L}(\tau, \hbar)$ be the fundamental solution given by hypergeometric modification (9).

$$
\widetilde{L}(\tau, \hbar)=e^{\tau / \hbar} \widetilde{T}(x, \hbar, \lambda)=e^{\tau / \hbar} \sum_{\boldsymbol{m} \geq 0, n \in \mathbb{Z}} \widetilde{T}_{\boldsymbol{m}, n}(\lambda) x^{\boldsymbol{m}} \hbar^{-n}
$$

Lemma 4.2. There exists a positive constant $C_{3}(\lambda)$ depending continuously on $\lambda$ such that

$$
\left\|\widetilde{T}_{\boldsymbol{m}, n}(\lambda)\right\| \leq C_{1} C_{3}(\lambda)^{|\boldsymbol{m}|+|n|} \begin{cases}1 / n ! & n \geq 0 \\ |n| ! & n \leq 0\end{cases}
$$

Here we set $0 !=1$. Moreover, $\widetilde{T}_{\boldsymbol{m}, n}=0$ for $\left.-n\right\rangle\langle\rho, \boldsymbol{m}\rangle$.

Proof. Because $\rho$ is nef, we have

$$
\sum_{n} \widetilde{T}_{\boldsymbol{m}, n}(\lambda) \hbar^{-n}=\prod_{k=1}^{\langle\rho, \boldsymbol{m}\rangle}(\rho+\lambda+k \hbar) \sum_{n \geq 0} T_{\boldsymbol{m}, n} \hbar^{-n}
$$


Therefore, by Lemma 4.1, we have

$$
\begin{aligned}
\left\|\widetilde{T}_{\boldsymbol{m}, n}(\lambda)\right\| & =\left\|\sum_{0 \leq l \leq\langle\rho, \boldsymbol{m}\rangle} \sum_{1 \leq k_{1}<k_{2}<\cdots<k_{l} \leq\langle\rho, \boldsymbol{m}\rangle}(\rho+\lambda)^{\langle\rho, \boldsymbol{m}\rangle-l} k_{1} k_{2} \cdots k_{l} T_{\boldsymbol{m}, n+l}\right\| \\
& \leq \sum_{0 \leq l \leq\langle\rho, \boldsymbol{m}\rangle}\|(\rho+\lambda)\|^{\langle\rho, \boldsymbol{m}\rangle-l}\left(\begin{array}{c}
\langle\rho, \boldsymbol{m}\rangle \\
l
\end{array}\right)(\langle\rho, \boldsymbol{m}\rangle-l+1) \cdots\langle\rho, \boldsymbol{m}\rangle C_{1} C_{2}^{|\boldsymbol{m}|+n+l} \frac{1}{(n+l) !} \\
& \leq C_{1} C_{2}^{|\boldsymbol{m}|+n} 2^{\langle\rho, \boldsymbol{m}\rangle} \sum_{0 \leq l \leq\langle\rho, \boldsymbol{m}\rangle}\left(\begin{array}{c}
\langle\rho, \boldsymbol{m}\rangle \\
l
\end{array}\right)\|(\rho+\lambda)\|^{\langle\rho, \boldsymbol{m}\rangle-l} C_{2}^{l} \frac{l !}{(n+l) !} \\
& \leq C_{1} C_{2}^{|\boldsymbol{m}|+|n|} 2^{\langle\rho, \boldsymbol{m}\rangle}\left(\|\rho+\lambda\|+2 C_{2}\right)^{\langle\rho, \boldsymbol{m}\rangle} \begin{cases}1 / n ! & n \geq 0, \\
|n| ! & n \leq 0 .\end{cases}
\end{aligned}
$$

Thus, we obtain the estimate. The latter part follows from (14).

Let $\mathcal{O}_{\lambda}^{\hbar, \hbar^{-1}}(\rho)$ be the set of formal power series of the form $\sum_{\boldsymbol{m} \geq 0, n \in \mathbb{Z}} A_{\boldsymbol{m}, n}(\lambda) x^{\boldsymbol{m}} \hbar^{-n}$ in $\mathbb{C}(\lambda)\left[\hbar, \hbar^{-1}\right] \llbracket x \rrbracket$ satisfying the following conditions:

(i) $A_{\boldsymbol{m}, n}=0$ for $\left.-n\right\rangle\langle\rho, \boldsymbol{m}\rangle$.

(ii) There exist positive continuous functions $B(\lambda), C(\lambda)$ defined on the complement of a finite subset of $\mathbb{C}$ such that

$$
\left|A_{\boldsymbol{m}, n}(\lambda)\right| \leq B(\lambda) C(\lambda)^{|\boldsymbol{m}|+|n|} \begin{cases}1 / n ! & n \geq 0 \\ |n| ! & n \leq 0 .\end{cases}
$$

The above lemma says that each matrix element of $\widetilde{T}(x, \hbar, \lambda)$ is contained in $\mathcal{O}_{\lambda}^{\hbar, \hbar^{-1}}(\rho)$.

Lemma 4.3. The set $\mathcal{O}_{\lambda}^{\hbar, \hbar^{-1}}(\rho)$ is a subring of $\mathbb{C}(\lambda)\left[\hbar, \hbar^{-1}\right] \llbracket x \rrbracket$.

Proof. We must check that $\mathcal{O}_{\lambda}^{\hbar, \hbar^{-1}}(\rho)$ is closed under product. Let $\sum_{\boldsymbol{m} \geq 0, n} A_{\boldsymbol{m}, n} x^{\boldsymbol{m}} \hbar^{-n}$, $\sum_{\boldsymbol{m} \geq 0, n} B_{\boldsymbol{m}, n} x^{\boldsymbol{m}} \hbar^{-n}$ be in $\mathcal{O}_{\lambda}^{\hbar, \hbar^{-1}}(\rho)$. By taking the maximum if necessary, we can assume that these two elements are estimated by the same functions $B(\lambda), C(\lambda)>1$. Set $C_{\boldsymbol{m}, n}=$ $\sum_{\boldsymbol{m}_{1}+\boldsymbol{m}_{2}=\boldsymbol{m}} \sum_{n_{1}+n_{2}=n} A_{\boldsymbol{m}_{1}, n_{1}} B_{\boldsymbol{m}_{2}, n_{2}}$. When $n \geq 0$, we have

$$
\begin{aligned}
\left|C_{\boldsymbol{m}, n}\right| & \leq B(\lambda)^{2} C(\lambda)^{|\boldsymbol{m}|+|n|}\left(\sum_{\substack{n_{1}+n_{2}=n, n_{i} \geq 0}} \frac{1}{n_{1} ! n_{2} !}+2 \sum_{i=1}^{\langle\rho, \boldsymbol{m}\rangle} C(\lambda)^{2 i} \frac{i !}{(n+i) !}\right) \sum_{\boldsymbol{m}_{1}+\boldsymbol{m}_{2}=\boldsymbol{m}} 1 \\
& \leq B(\lambda)^{2} C(\lambda)^{|\boldsymbol{m}|+|n|} \frac{1}{n !}\left(2^{n}+2 \frac{C(\lambda)^{2\langle\rho, \boldsymbol{m}\rangle+2}}{C(\lambda)^{2}-1}\right) C^{\prime|\boldsymbol{m}|}
\end{aligned}
$$

for some $C^{\prime}>0$. When $n \leq 0$, we have

$$
\begin{aligned}
\left|C_{\boldsymbol{m}, n}\right| & \leq B(\lambda)^{2} C(\lambda)^{|\boldsymbol{m}|+|n|}\left(\sum_{\substack{n_{1}+n_{2}=n, n_{i} \leq 0}}\left|n_{1}\right| !\left|n_{2}\right| !+2 \sum_{i=1}^{\langle\rho, \boldsymbol{m}\rangle-|n|} C(\lambda)^{2 i} \frac{(i+|n|) !}{i !}\right) \sum_{\boldsymbol{m}_{1}+\boldsymbol{m}_{2}=\boldsymbol{m}} 1 \\
& \leq B(\lambda)^{2} C(\lambda)^{|\boldsymbol{m}|+|n|}|n| !\left(\langle\rho, \boldsymbol{m}\rangle+1+2\left(1+C(\lambda)^{2}\right)^{\langle\rho, \boldsymbol{m}\rangle}\right) C^{\prime|\boldsymbol{m}|}
\end{aligned}
$$

for some $C^{\prime}>0$. Therefore, we have the desired estimate for the product.

Let $\Omega_{j}(x, \hbar, \lambda)$ be the connection matrix of $\widetilde{\nabla}^{\hbar}$ given in (10). Let $\langle\langle\cdot, \cdot\rangle\rangle$ be the bilinear form defined in Proposition 3.4 (ii). 
Lemma 4.4. The elements of the form $\langle\langle\alpha, \beta\rangle\rangle,\left\langle\left\langle\alpha, \Omega_{j} \beta\right\rangle\right\rangle$ are contained in both $\mathbb{C}[\hbar, \lambda] \llbracket x \rrbracket$ and $\mathcal{O}_{\lambda}^{\hbar, \hbar^{-1}}(\rho)$ for $\alpha, \beta \in H^{*}(X)$.

Proof. By Proposition 3.4 , we can see that $\langle\langle\alpha, \beta\rangle\rangle$ and $\left\langle\left\langle\alpha, \Omega_{j} \beta\right\rangle\right\rangle$ are in $\mathbb{C}[\hbar, \lambda] \llbracket x \rrbracket$. Since we have

$$
\langle\langle\alpha, \beta\rangle\rangle=\langle\widetilde{T}(x,-\hbar, \lambda) \alpha, \widetilde{T}(x, \hbar, \lambda) \beta\rangle_{S^{1}}^{\mathcal{L}},
$$

we can see that $\langle\langle\alpha, \beta\rangle\rangle$ is in $\mathcal{O}_{\lambda}^{\hbar, \hbar^{-1}}(\rho)$ by Lemma 4.2 On the other hand, we have

$$
\begin{aligned}
\left\langle\left\langle\alpha, \Omega_{j} \beta\right\rangle\right\rangle & =\left\langle\widetilde{L}(\tau,-\hbar) \alpha, \hbar \frac{\partial}{\partial t_{j}} \widetilde{L}(\tau,-\hbar) \beta\right\rangle_{S^{1}}^{\mathcal{L}} \\
& =\left\langle\widetilde{T}(x,-\hbar, \lambda) \alpha, \nabla_{j}^{\mathrm{cl}} \widetilde{T}(x, \hbar, \lambda) \beta\right\rangle_{S^{1}}^{\mathcal{L}},
\end{aligned}
$$

where $\nabla_{j}^{\mathrm{cl}}=\hbar \partial_{t_{j}}+p_{j}$. Therefore, $\left\langle\left\langle\alpha, \Omega_{j} \beta\right\rangle\right\rangle$ is also in $\mathcal{O}_{\lambda}^{\hbar, \hbar^{-1}}(\rho)$ by Lemma 4.2

Define another ring $\mathcal{O}_{\lambda}^{\hbar}(\rho)$ by

$$
\mathcal{O}_{\lambda}^{\hbar}(\rho)=\mathcal{O}_{\lambda}^{\hbar, \hbar^{-1}}(\rho) \cap \mathbb{C}(\lambda)[\hbar] \llbracket x \rrbracket .
$$

By the above lemma, $\langle\langle\alpha, \beta\rangle\rangle,\left\langle\left\langle\alpha, \Omega_{j} \beta\right\rangle\right\rangle$ are contained in $\mathcal{O}_{\lambda}^{\hbar}(\rho)$.

Lemma 4.5. The ring $\mathcal{O}_{\lambda}^{\hbar}(\rho)$ is a local ring.

Proof. Let $\sum_{\boldsymbol{m} \geq 0} \sum_{n=0}^{\langle\rho, \boldsymbol{m}\rangle} A_{\boldsymbol{m}, n}(\lambda) x^{\boldsymbol{m}} \hbar^{n}$ be in $\mathcal{O}_{\lambda}^{\hbar}(\rho)$. We will show that if $A_{0,0}(\lambda)=1$, this element is invertible in $\mathcal{O}_{\lambda}^{\hbar}(\rho)$. We can assume that $\left|A_{\boldsymbol{m}, n}\right| \leq B(\lambda) C(\lambda)^{|\boldsymbol{m}|+n} n$ ! for some positive functions $B(\lambda), C(\lambda)>1$. Set $1+\sum_{\boldsymbol{m}>0, n \geq 0} B_{\boldsymbol{m}, n} x^{\boldsymbol{m}} \hbar^{n}=\left(1+\sum_{\boldsymbol{m}>0, n \geq 0} A_{\boldsymbol{m}, n} x^{\boldsymbol{m}} \hbar^{n}\right)^{-1}$. Then we have

$$
\begin{aligned}
& \left|B_{\boldsymbol{m}, n}\right|=\left|\sum_{l \geq 1}(-1)^{l} \sum_{\substack{\boldsymbol{m}=\boldsymbol{m}_{1}+\cdots+\boldsymbol{m}_{l} \\
\boldsymbol{m}_{i}>0}} \sum_{\substack{n=n_{1}+\cdots+n_{l} \\
0 \leq n_{i} \leq\left\langle\rho, \boldsymbol{m}_{i}\right\rangle}} A_{\boldsymbol{m}_{1}, n_{1}} \cdots A_{\boldsymbol{m}_{l}, n_{l}}\right| \\
& \leq \sum_{l \geq 1} \sum_{\substack{\boldsymbol{m}=\boldsymbol{m}_{1}+\cdots+\boldsymbol{m}_{l} \\
\boldsymbol{m}_{i}>0}} \sum_{\substack{n=n_{1}+\cdots+n_{l} \\
0 \leq n_{i} \leq\left\langle\rho, \boldsymbol{m}_{i}\right\rangle}} B(\lambda)^{l} C(\lambda)^{|\boldsymbol{m}|+n} n_{1} ! \cdots n_{l} \text { ! } \\
& \leq B(\lambda)^{|\boldsymbol{m}|} C(\lambda)^{|\boldsymbol{m}|+n} n ! \sum_{l \geq 1} \sum_{\substack{\boldsymbol{m}=\boldsymbol{m}_{1}+\cdots+\boldsymbol{m}_{l} \\
\boldsymbol{m}_{i}>0}}\left(\begin{array}{c}
n+l-1 \\
n
\end{array}\right) \\
& \leq B(\lambda)^{|\boldsymbol{m}|} C(\lambda)^{|\boldsymbol{m}|+n} 2^{n+|\boldsymbol{m}|-1} n ! \sum_{l \geq 1} \sum_{\substack{\boldsymbol{m}=\boldsymbol{m}_{1}+\cdots+\boldsymbol{m}_{l} \\
\boldsymbol{m}_{i}>0}} 1,
\end{aligned}
$$

where we used $l \leq|\boldsymbol{m}|$. The summation factor is of exponential order in $|\boldsymbol{m}|$ because

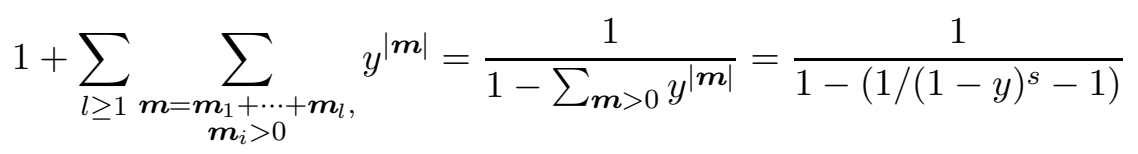

is analytic around $y=0$.

Proposition 4.6. Each entry of the connection matrix $\Omega_{j}(x, \hbar, \lambda)$ is contained in the ring $\mathcal{O}_{\lambda}^{\hbar}(\rho)$. 
Proof. By Lemma 4.4 $\eta_{k l}=\left\langle\left\langle p_{k}, p_{l}\right\rangle\right\rangle$ and $\left\langle\left\langle p_{k}, \Omega_{j} p_{l}\right\rangle\right\rangle$ belong to $\mathcal{O}_{\lambda}^{\hbar}(\rho)$. It is easy to see that $\lim _{q \rightarrow 0} \eta_{k l}=\left\langle p_{k}, p_{l}\right\rangle_{S^{1}}^{\mathcal{L}}$. Because the matrix $\left(\left\langle p_{k}, p_{l}\right\rangle_{S^{1}}^{\mathcal{L}}\right)$ is invertible in $\operatorname{Mat}(s+1, \mathbb{C}(\lambda))$ and $\mathcal{O}_{\lambda}^{\hbar}(\rho)$ is a local ring, the matrix $\left(\eta_{k l}\right)$ is invertible in $\operatorname{Mat}\left(s+1, \mathcal{O}_{\lambda}^{\hbar}(\rho)\right)$. Therefore, matrix elements $\Omega_{j ; k l}=\sum_{i} \eta^{k i}\left\langle\left\langle p_{i}, \Omega_{j} p_{l}\right\rangle\right\rangle$ are in $\mathcal{O}_{\lambda}^{\hbar}(\rho)$.

4.2. Gauge fixing. In this subsection, we will find a gauge transformation $g_{2}$ which changes the connection matrices into $\hbar$-independent ones.

Let $\mathcal{O}_{\lambda}^{\hbar}$ be the set of formal power series $\sum_{\boldsymbol{m}, n \geq 0} A_{\boldsymbol{m}, n}(\lambda) x^{\boldsymbol{m}} \hbar^{n}$ in $\mathbb{C}(\lambda)[\hbar] \llbracket x \rrbracket$ satisfying the following conditions:

(i) $A_{\boldsymbol{m}, n}=0$ if $|\boldsymbol{m}|_{0}=0, n>0$,

(ii) There exists positive continuous functions $B(\lambda)$ and $C(\lambda)$ defined on the complement of a finite subset of $\mathbb{C}$ such that

$$
\left|A_{\boldsymbol{m}, n}(\lambda)\right| \leq B(\lambda) C(\lambda)^{|\boldsymbol{m}|+n}|\boldsymbol{m}|_{0}^{n},
$$

where $|\boldsymbol{m}|_{0}=\sum_{a=1}^{r} m_{a}(\leq|\boldsymbol{m}|)$. It is easy to see that $\mathcal{O}_{\lambda}^{\hbar}(\rho)$ is contained in $\mathcal{O}_{\lambda}^{\hbar}$.

Lemma 4.7. $\mathcal{O}_{\lambda}^{\hbar}$ is a local ring.

We omit the proof because it is similar to Lemma 4.5.

Proposition 4.8. There exists a unique gauge transformation $g_{2}$ with entries in $\mathcal{O}_{\lambda}^{\hbar}$ such that $\left.g_{2}\right|_{q=0}=\mathrm{id}$ and that the new connection matrix $\widehat{\Omega}_{j}$ of $g_{2}^{*} \widetilde{\nabla}^{\hbar}=\hbar d+\sum_{j=0}^{s} \widehat{\Omega}_{j} d t_{j}$ is $\hbar$-independent. Moreover, $\widehat{\Omega}_{j}$ is convergent.

This proposition is considered to be a general gauge fixing lemma. It is applicable to any flat connection of the form $\hbar d+\Omega$ which is defined over $\mathcal{O}_{\lambda}^{\hbar}$, regular singular along $q_{1} q_{2} \cdots q_{r}=0$ and whose residue matrices at $q=0$ are nilpotent. In [17, Theorem 4.6, we showed the existence and the uniqueness of $g_{2}$ in $\operatorname{End}\left(H^{*}(X)\right) \otimes \mathbb{C}[\hbar, \lambda] \llbracket x \rrbracket$ by using a formal Birkhoff factorization. Here, we will show that $g_{2}$ also belongs to $\operatorname{End}\left(H^{*}(X)\right) \otimes \mathcal{O}_{\lambda}^{\hbar}$. This gauge fixing can be considered as a procedure of renormalization. A divergent connection can be renormalized by $g_{2}$ to yield a finite (convergent) result.

Proof. Once we establish the existence of $g_{2}$ in $\operatorname{End}\left(H^{*}(X)\right) \otimes \mathcal{O}_{\lambda}^{\hbar}$, we can see that $\widehat{\Omega}_{j}$ is convergent because it is contained in $\operatorname{End}\left(H^{*}(X)\right) \otimes \mathcal{O}_{\lambda}^{\hbar}$ and $\hbar$-independent at the same time. Thus, it suffices to solve for $g_{2}$ in $\operatorname{End}\left(H^{*}(X)\right) \otimes \mathcal{O}_{\lambda}^{\hbar}$. Set $\Omega_{j}=\sum_{\boldsymbol{d}, n \geq 0} \Omega_{j ; \boldsymbol{d}, n}\left(t_{r+1}, \cdots, t_{s}, \lambda\right) q^{\boldsymbol{d}} \hbar^{n}$. Because $\Omega_{j}$ is defined over $\mathcal{O}_{\lambda}^{\hbar}$, there exist a neighborhood $U \subset \mathbb{C}^{s-r}$ of 0 and continuous functions $B(\lambda), C(\lambda)>0$ such that $\left\|\Omega_{j ; \boldsymbol{d}, n}(t, \lambda)\right\| \leq B(\lambda) C(\lambda)^{|\boldsymbol{d}|+n}|\boldsymbol{d}|^{n}$ for $\left(t_{r+1}, \cdots, t_{s}\right) \in U$ and $0 \leq j \leq s$. From now on, we omit $\lambda$ and $\left(t_{r+1}, \cdots, t_{s}\right)$ in the notation, but $\left(t_{r+1}, \cdots, t_{s}\right)$ is always assumed to be in $U$. We set $g_{2}=\sum_{\boldsymbol{d}, n \geq 0} G_{\boldsymbol{d}, n} q^{\boldsymbol{d}} \hbar^{n}, \widehat{\Omega}_{j}=\sum_{\boldsymbol{d}, n \geq 0} \widehat{\Omega}_{j ; \boldsymbol{d}} q^{\boldsymbol{d}}$. By expanding the relation

$$
g_{2} \widehat{\Omega}_{a}=\Omega_{a} g_{2}+\hbar q_{a} \frac{\partial g_{2}}{\partial q_{a}}, \quad 1 \leq a \leq r
$$

we obtain the following equations:

$$
\begin{aligned}
& d_{a} G_{\boldsymbol{d}, n-1}+\operatorname{ad}\left(p_{a}\right) G_{\boldsymbol{d}, n}=\sum_{\substack{\boldsymbol{d}_{1}+\boldsymbol{d}_{2}=\boldsymbol{d}, \boldsymbol{d}_{1}>0, \boldsymbol{d}_{2}>0}} G_{\boldsymbol{d}_{2}, n} \widehat{\Omega}_{a ; \boldsymbol{d}_{1}}-\sum_{\substack{\boldsymbol{d}_{1}+\boldsymbol{d}_{2}=\boldsymbol{d}, n=n_{1}+n_{2} \\
\boldsymbol{d}_{1}>0}} \Omega_{a ; \boldsymbol{d}_{1}, n_{1}} G_{\boldsymbol{d}_{2}, n_{2}} \quad(n \geq 1), \\
& \operatorname{ad}\left(p_{a}\right) G_{\boldsymbol{d}, 0}=\widehat{\Omega}_{a ; \boldsymbol{d}}+\sum_{\substack{\boldsymbol{d}_{1}+\boldsymbol{d}_{2}=\boldsymbol{d}, \boldsymbol{d}_{1}>0, \boldsymbol{d}_{2}>0}} G_{\boldsymbol{d}_{2}, 0} \widehat{\Omega}_{a ; \boldsymbol{d}_{1}}-\sum_{\substack{\boldsymbol{d}_{1}+\boldsymbol{d}_{2}=\boldsymbol{d}, \boldsymbol{d}_{1}>0}} \Omega_{a ; \boldsymbol{d}_{1}, 0} G_{\boldsymbol{d}_{2}, 0},
\end{aligned}
$$


where we used $G_{0, n}=\delta_{0, n}, \widehat{\Omega}_{a ; 0}=p_{a}$ and $\Omega_{a ; 0, n}=\delta_{0, n} p_{a}$. Note that $\Omega_{a ; \boldsymbol{d}, n}$ is known and $G_{\boldsymbol{d}, n}$ and $\widehat{\Omega}_{a ; \boldsymbol{d}}$ are unknown. Assume by induction that we know $G_{\boldsymbol{d}^{\prime}, n}$ and $\widehat{\Omega}_{a ; \boldsymbol{d}^{\prime}}$ for all $\boldsymbol{d}^{\prime}$ with $\left|\boldsymbol{d}^{\prime}\right|<\bar{d}$. For a multi-index $\boldsymbol{d}$ with $|\boldsymbol{d}|=\bar{d}$, we first solve for $G_{\boldsymbol{d}, n}$ for all $n \geq 0$ by using (15), and then we solve for $\widehat{\Omega}_{a ; \boldsymbol{d}}$ by using (16).

More precisely, we must solve for $G_{\boldsymbol{d}, n}, \widehat{\Omega}_{a ; \boldsymbol{d}}$ with estimates. Introduce the following notation:

$$
\begin{gathered}
g_{d, n}=\frac{1}{d^{n}} \sum_{|\boldsymbol{d}|=d}\left\|G_{\boldsymbol{d}, n}\right\|, \quad \omega_{a ; d, n}=\frac{1}{d^{n}} \sum_{|\boldsymbol{d}|=d}\left\|\Omega_{a ; \boldsymbol{d}, n}\right\|, \quad \widehat{\omega}_{a ; d}=\sum_{|\boldsymbol{d}|=d}\left\|\widehat{\Omega}_{a ; \boldsymbol{d}}\right\|, \\
H_{a ; \boldsymbol{d}, n}=\text { the right hand side of (15) }, \quad h_{d, n}=\max _{1 \leq a \leq r} \frac{1}{d^{n}} \sum_{|\boldsymbol{d}|=d}\left\|H_{a ; \boldsymbol{d}, n}\right\| \quad(n \geq 1) .
\end{gathered}
$$

There exist positive $A_{1}, B_{1}$ such that $\omega_{a ; d, n} \leq A_{1} B_{1}^{d+n}$. Assume by induction that

$$
g_{d, n} \leq \frac{B_{2}^{d}}{(d+1)^{M}} B_{3}^{n}, \quad \widehat{\omega}_{a ; d} \leq A_{2} \frac{B_{2}^{d}}{(d+1)^{M}} \quad(1 \leq a \leq r)
$$

hold for all $d<\bar{d}$. We take $A_{2}$ so that $\widehat{\omega}_{a ; 0}=\left\|p_{a}\right\| \leq A_{2}$ holds. Then (17) is valid for $d=0$ because $g_{0, n}=\delta_{0, n}$. We will specify $B_{2}, B_{3}, M$ later. Take a $\boldsymbol{d}$ with $|\boldsymbol{d}|=\bar{d}$. Let $a(\boldsymbol{d})$ be an index such that $d_{a(\boldsymbol{d})}=\max \left\{d_{1}, \ldots, d_{r}\right\}$. Let $C$ be a constant satisfying $\left\|\operatorname{ad}\left(p_{a}\right)\right\| \leq C$ for $1 \leq a \leq r$. By (15), we have

$$
\begin{aligned}
G_{\boldsymbol{d}, n} & =\frac{1}{d_{a(\boldsymbol{d})}}\left(H_{a(\boldsymbol{d}) ; \boldsymbol{d}, n+1}-\operatorname{ad}\left(p_{a(\boldsymbol{d})}\right) G_{\boldsymbol{d}, n+1}\right) \\
& =\frac{1}{d_{a(\boldsymbol{d})}} H_{a(\boldsymbol{d}) ; \boldsymbol{d}, n+1}-\frac{\operatorname{ad}\left(p_{a(\boldsymbol{d})}\right)}{d_{a(\boldsymbol{d})}^{2}} H_{a(\boldsymbol{d}) ; \boldsymbol{d}, n+2}+\cdots+\frac{\operatorname{ad}\left(p_{a(\boldsymbol{d})}\right)^{2 N}}{d_{a(\boldsymbol{d})}^{2 N+1}} H_{a(\boldsymbol{d}) ; \boldsymbol{d}, n+2 N+1},
\end{aligned}
$$

where $N=\operatorname{dim}_{\mathbb{C}} X$ and we used $\operatorname{ad}\left(p_{a(\boldsymbol{d})}\right)^{2 N+1}=0$. By using $\left(|\boldsymbol{d}| / d_{a(\boldsymbol{d})}\right) \leq r$, we have

$$
g_{\bar{d}, n} \leq r h_{\bar{d}, n+1}+r^{2} C h_{\bar{d}, n+2}+\cdots+r^{2 N+1} C^{2 N} h_{\bar{d}, n+2 N+1} .
$$

Also we have

$$
\begin{aligned}
h_{\bar{d}, n} & \leq \max _{1 \leq a \leq r}\left(\sum_{\substack{\left|\boldsymbol{d}_{1}\right|+\left|\boldsymbol{d}_{2}\right|=\bar{d}, \boldsymbol{d}_{1}>0, \boldsymbol{d}_{2}>0}} \frac{1}{\bar{d}^{n}}\left\|G_{\boldsymbol{d}_{2}, n}\right\|\left\|\widehat{\Omega}_{a ; \boldsymbol{d}_{1}}\right\|+\sum_{\begin{array}{c}
\left|\boldsymbol{d}_{1}\right|+\left|\boldsymbol{d}_{2}\right|=\bar{d}, \\
n_{1}+n_{2}=n, \boldsymbol{d}_{1}>0
\end{array}} \frac{1}{\bar{d}^{n_{1}}}\left\|\Omega_{a ; \boldsymbol{d}_{1}, n_{1}}\right\| \frac{1}{\bar{d}^{n_{2}}}\left\|G_{\boldsymbol{d}_{2}, n_{2}}\right\|\right) \\
& \leq \max _{1 \leq a \leq r}\left(\sum_{d_{1}=1}^{\bar{d}-1} g_{\bar{d}-d_{1}, n} \widehat{\omega}_{a ; d_{1}}+\sum_{d_{1}=1}^{\bar{d}} \sum_{k=0}^{n} \omega_{a ; d_{1}, k} g_{\bar{d}-d_{1}, n-k}\right) .
\end{aligned}
$$

By using (16), we have

$$
\begin{aligned}
\widehat{\omega}_{a ; \bar{d}} & \leq C \sum_{|\boldsymbol{d}|=\bar{d}}\left\|G_{\boldsymbol{d}, 0}\right\|+\sum_{\begin{array}{c}
\left|\boldsymbol{d}_{1}\right|+\left|\boldsymbol{d}_{2}\right|=\bar{d} \\
\boldsymbol{d}_{1}>0, \boldsymbol{d}_{2}>0
\end{array}}\left\|G_{\boldsymbol{d}_{2}, 0}\right\|\left\|\widehat{\Omega}_{a ; \boldsymbol{d}_{1}}\right\|+\sum_{\substack{\left|\boldsymbol{d}_{1}\right|+\left|\boldsymbol{d}_{2}\right|=\bar{d} \\
\boldsymbol{d}_{1}>0}}\left\|\Omega_{a ; \boldsymbol{d}_{1}, 0}\right\|\left\|G_{\boldsymbol{d}_{2}, 0}\right\| \\
& \leq C g_{\bar{d}, 0}+\sum_{d_{1}=1}^{\bar{d}-1} g_{\bar{d}-d_{1}, 0} \widehat{\omega}_{a ; d_{1}}+\sum_{d_{1}=1}^{\bar{d}} \omega_{a ; d_{1}, 0} g_{\bar{d}-d_{1}, 0} .
\end{aligned}
$$


By (19) and the assumption (17), we obtain

$$
\begin{aligned}
h_{\bar{d}, n} & \leq A_{2} B_{2}^{\bar{d}} B_{3}^{n} \sum_{i=1}^{\bar{d}-1} \frac{1}{(\bar{d}-i+1)^{M}(i+1)^{M}}+A_{1} B_{2}^{\bar{d}} B_{3}^{n} \sum_{i=1}^{\bar{d}} \sum_{k=0}^{n}\left(\frac{B_{1}}{B_{2}}\right)^{i}\left(\frac{B_{1}}{B_{3}}\right)^{k} \frac{1}{(\bar{d}-i+1)^{M}} \\
& \leq\left(A_{2} \varepsilon_{1}(M)+\frac{A_{1}}{1-B_{1} / B_{3}} \varepsilon_{2}\left(B_{2}, M\right)\right) \frac{B_{2}^{\bar{d}}}{(\bar{d}+1)^{M}} B_{3}^{n},
\end{aligned}
$$

where we set

$$
\varepsilon_{1}(M)=\sup _{\bar{d} \geq 1} \sum_{i=1}^{\bar{d}-1} \frac{(\bar{d}+1)^{M}}{(\bar{d}-i+1)^{M}(i+1)^{M}}, \quad \varepsilon_{2}\left(B_{2}, M\right)=\sup _{\bar{d} \geq 1} \sum_{i=1}^{\bar{d}}\left(\frac{B_{1}}{B_{2}}\right)^{i} \frac{(\bar{d}+1)^{M}}{(\bar{d}-i+1)^{M}} .
$$

By (18) and (211), we obtain

$$
g_{\bar{d}, n} \leq \varepsilon_{3}\left(B_{2}, B_{3}, M\right) \frac{B_{2}^{\bar{d}}}{(\bar{d}+1)^{M}} B_{3}^{n},
$$

where we set

$$
\varepsilon_{3}\left(B_{2}, B_{3}, M\right)=\left(A_{2} \varepsilon_{1}(M)+\frac{A_{1}}{1-B_{1} / B_{3}} \varepsilon_{2}\left(B_{2}, M\right)\right) r B_{3} \frac{\left(r B_{3} C\right)^{2 N+1}-1}{r B_{3} C-1} .
$$

By (22) and (20), we have

$$
\widehat{\omega}_{a ; \bar{d}} \leq \varepsilon_{3}\left(B_{2}, B_{3}, M\right)\left(C+A_{2} \varepsilon_{1}(M)+A_{1} \varepsilon_{2}\left(B_{2}, M\right)\right) \frac{B_{2}^{\bar{d}}}{(\bar{d}+1)^{M}} .
$$

In order to complete the induction step, we need to specify the parameters $B_{2}, B_{3}, M$. First we set $B_{3}=2 B_{1}$. To choose $B_{2}$ and $M$, we need the following lemma:

Lemma 4.9. $\lim _{M \rightarrow \infty} \varepsilon_{1}(M)=0, \lim _{B_{2} \rightarrow \infty} \varepsilon_{2}\left(B_{2}, M\right)=0$.

The proof will be given in the Appendix. For sufficiently large $M$, we have

$$
A_{2} \varepsilon_{1}(M) r B_{3} \frac{\left(r B_{3} C\right)^{2 N+1}-1}{r B_{3} C-1}<\min \left\{\frac{1}{2}, \frac{A_{2}}{3 C}\right\} \text { and } \varepsilon_{1}(M)<\frac{1}{3} .
$$

Next, for sufficiently large $B_{2}$, we have

$$
\frac{A_{1}}{1-B_{1} / B_{3}} \varepsilon_{2}\left(B_{2}, M\right) r B_{3} \frac{\left(r B_{3} C\right)^{2 N+1}-1}{r B_{3} C-1}<\frac{1}{2} \text { and } A_{1} \varepsilon_{2}\left(B_{2}, M\right)<\frac{A_{2}}{3} .
$$

Now, it is easy to check that $\varepsilon_{3}\left(B_{2}, B_{3}, M\right)<1$ and $\varepsilon_{3}\left(B_{2}, B_{3}, M\right)\left(C+A_{2} \varepsilon_{1}(M)+A_{1} \varepsilon_{2}\left(B_{2}, M\right)\right)<$ $A_{2}$. Therefore, by (22) and (23), we complete the induction step.

Proof of Theorem 1.1. As explained at the end of the section 3] in order to obtain the structure constants of $Q H_{S^{1}}^{*}(X, \mathcal{L})$ from $g_{2}^{*} \widetilde{\nabla}^{\hbar}$, it suffices to find a new coordinate system $\hat{x}=\left\{\hat{t}_{0}, \hat{q}_{1}=\right.$ $\left.\exp \left(\hat{t}_{1}\right), \ldots, \hat{q}_{r}=\exp \left(\hat{t}_{r}\right), \hat{t}_{r+1}, \ldots, \hat{t}_{s}\right\}$ such that the connection matrix $\widehat{\Omega}_{\hat{j}}$ defined by $g_{2}^{*} \widetilde{\nabla}^{\hbar}=$ $\hbar d+\sum_{j=0}^{s} \widehat{\Omega}_{j} d t_{j}=\hbar d+\sum_{j=0} \widehat{\Omega}_{\hat{j}} d \hat{t}_{j}$ satisfies $\widehat{\Omega}_{\hat{j}} 1=p_{j}$. Then $\widehat{\Omega}_{\hat{j}}$ gives the twisted quantum product $p_{j} * \mathcal{L}$. Because $\widehat{\Omega}_{j}$ is already convergent, new coordinates $\hat{x}$ also become convergent functions in $x$. 


\section{MirRoR SYMmetrY FOR NON-NEF TORIC VARIETIES}

In this section, we will study mirror symmetry and the quantum cohomology of a not necessarily nef toric variety. In [17, we showed that the quantum $D$-module of $Q D M^{*}(X)$ of a toric variety $X$ can be reconstructed from the equivariant Floer cohomology $F H_{S^{1}}^{*}$ [7, 16] by a generalized mirror transformation. There, the quantum $D$-module $Q D M^{*}(X)$ was described in terms of hypergeometric series ( $I$-function). Here, we describe $Q D M^{*}(X)$ in terms of the mirror oscillatory integral introduced by Givental [8, 10. The oscillatory integral satisfies (a generalized version of) the Mellin system of hypergeometric differential equations. We will show that the equivariant Floer cohomology $F H_{S^{1}}^{*}$ is isomorphic to the q-adic completion of the Mellin system. Then, results in [17 can be restated as $Q D M^{*}(X)$ restricted to some non-linear subspace of $H^{*}(X)$ is isomorphic to the completion of the Mellin system. Using a method similar to section [4 we will show the convergence of the quantum cohomology of toric varieties. By using mirror symmetry, we will also show the semisimplicity.

5.1. Mirrors and the Mellin system. Let $X$ be a smooth projective toric variety defined by a fan $\Sigma \subset \mathbb{R}^{N}$. Take a nef integral basis $\left\{p_{1}, \ldots, p_{r}\right\}$ of $H^{2}(X, \mathbb{Z})$. Let $D_{1}, \ldots, D_{r+N}$ be all the torus invariant prime divisors and $w_{1}, \ldots, w_{r+N} \in H^{2}(X, \mathbb{Z})$ be their Poincaré duals. We write $w_{i}=\sum_{a=1}^{r} m_{i a} p_{a}$. We can recover $X$ from the data $w_{i}$ and Kähler class $\eta_{X}$ in $H^{2}(X, \mathbb{R})$. Set $\mathcal{B}=\left\{I \subset\{1, \ldots, N+r\} \mid \eta_{X} \notin \sum_{i \in I} \mathbb{R}_{>0} w_{i}\right\}$. Then we have

$$
X=\mathbb{C}_{\mathcal{B}}^{r+N} /\left(\mathbb{C}^{*}\right)^{r}, \quad \mathbb{C}_{\mathcal{B}}^{r+N}:=\mathbb{C}^{r+N} \backslash \bigcup_{I \in \mathcal{B}}\left\{\left(z_{1}, \ldots, z_{r+N}\right) \mid z_{i}=0 \text { for } i \notin I\right\} .
$$

Here, $\left(\mathbb{C}^{*}\right)^{r}$ acts on $\mathbb{C}^{r+N}$ as $\left(z_{1}, \ldots, z_{r+N}\right) \mapsto\left(t^{w_{1}} z_{1}, \ldots, t^{w_{r+N}} z_{r+N}\right)$, where $t^{w_{i}}=\prod_{a=1}^{r} t_{a}^{m_{i a}}$. The divisor $D_{i}$ corresponds to $\left\{z_{i}=0\right\}$. Let $\mathrm{w}_{i}$ denotes a coordinate of the mirror corresponding to the class $w_{i}$. Let $\pi: Y=\left(\mathbb{C}^{*}\right)^{r+N} \rightarrow\left(\mathbb{C}^{*}\right)^{r}$ be a family of algebraic tori defined by $\pi\left(\mathrm{w}_{1}, \ldots, \mathrm{w}_{r+N}\right)=\left(q_{1}, \ldots, q_{r}\right), q_{a}=\prod_{i=1}^{r+N} \mathrm{w}_{i}^{m_{i a}}$. Define a function $F(\mathrm{w})$ on $Y$ as $F(\mathrm{w})=\mathrm{w}_{1}+\cdots+\mathrm{w}_{r+N}$. We write $Y_{q}=\pi^{-1}(q)$ and $F_{q}=\left.F\right|_{Y_{q}}$ for $q \in\left(\mathbb{C}^{*}\right)^{r}$. The mirror oscillatory integral is given by

$$
\mathcal{I}_{\Gamma}(q, \hbar)=\int_{\Gamma_{q} \subset Y_{q}} e^{F_{q} / \hbar} \omega_{q}
$$

where $\Gamma_{q}$ is a non-compact real $N$-cycle in $Y_{q}$ such that the integral converges. The holomorphic volume form $\omega_{q}$ is given below. Take coordinates $\left(s_{1}, \cdots, s_{N}\right)$ of fibers $Y_{q}$ of the form $s_{b}=$ $\prod_{i=1}^{r+N} \mathrm{w}_{i}^{m_{i b}^{\prime}}$, where the $(r+N) \times N$ matrix $\left(m_{i b}^{\prime}\right): \mathbb{Z}^{r+N} \rightarrow \mathbb{Z}^{N}$ gives a splitting of the exact sequence

$$
0 \longrightarrow \mathbb{Z}^{N} \stackrel{\operatorname{ker}\left(m_{i a}\right)}{\longrightarrow} \mathbb{Z}^{r+N} \stackrel{\left(m_{i a}\right)}{\longrightarrow} \mathbb{Z}^{r} \cong H^{2}(X, \mathbb{Z}) \longrightarrow 0 .
$$

By this splitting, we can write $\mathrm{w}_{i}=\prod_{a=1}^{r} q_{a}^{l_{a i}} \prod_{b=1}^{N} s_{b}^{x_{i b}}$. Here, $\vec{x}_{i}=\left(x_{i 1}, \ldots, x_{i N}\right) \in \mathbb{Z}^{N}$ gives the primitive generator of the $i$-th one dimensional cone of the fan $\Sigma$. The matrix $\left(l_{a i}\right): \mathbb{Z}^{r} \rightarrow \mathbb{Z}^{r+N}$ also gives a splitting of the exact sequence (24). We can choose this splitting so that $l_{a i} \geq 0$ because $\left\{p_{1}, \ldots, p_{r}\right\}$ is a nef basis. We define $\omega_{q}$ by

$$
\omega_{q}=\frac{d \log \mathrm{w}_{1} \wedge \cdots \wedge d \log \mathrm{w}_{r+N}}{d \log q_{1} \wedge \cdots \wedge d \log q_{r}}=\left.d \log s_{1} \wedge \cdots \wedge d \log s_{N}\right|_{Y_{q}} .
$$

This is independent of a choice of the splitting. 
Proposition 5.1. The oscillatory integral $\mathcal{I}_{\Gamma}(q, \hbar)$ satisfies $\mathcal{P}_{\boldsymbol{d}} \mathcal{I}_{\Gamma}(q, \hbar)=0$ for all $\boldsymbol{d} \in \mathbb{Z}^{r}$, where $\mathcal{P}_{\boldsymbol{d}}$ is a differential operator defined by

$$
\mathcal{P}_{\boldsymbol{d}}=q^{\boldsymbol{d}} \prod_{\left\langle w_{i}, \boldsymbol{d}\right\rangle<0} \prod_{k=0}^{-\left\langle w_{i}, \boldsymbol{d}\right\rangle-1}\left(\sum_{a=1}^{r} m_{i a} \hbar \partial_{a}-k \hbar\right)-\prod_{\left\langle w_{i}, \boldsymbol{d}\right\rangle>0} \prod_{k=0}^{\left\langle w_{i}, \boldsymbol{d}\right\rangle-1}\left(\sum_{a=1}^{r} m_{i a} \hbar \partial_{a}-k \hbar\right),
$$

where $\partial_{a}=q_{a} \partial / \partial q_{a}$.

This proposition may be well-known, but we include a proof for completeness.

Proof. First we have

$$
\begin{aligned}
\int_{\Gamma} \hbar \frac{\partial e^{F_{q} / \hbar}}{\partial \log \mathrm{w}_{i}} \omega_{q} & =\int_{\Gamma}\left(\sum_{a=1}^{r} m_{i a} \hbar \partial_{a} e^{F_{q} / \hbar}+\sum_{b=1}^{N} m_{i b}^{\prime} \hbar \frac{\partial e^{F_{q} / \hbar}}{\partial \log s_{b}}\right) \prod_{b} d \log s_{b} \\
& =\left(\sum_{a=1}^{r} m_{i a} \hbar \partial_{a}\right) \int_{\Gamma} e^{F_{q} / \hbar} \prod_{b} d \log s_{b}+\sum_{c=1}^{N} m_{i c}^{\prime} \int_{\Gamma} \hbar d\left(e^{F_{q} / \hbar} \prod_{b \neq c} d \log s_{b}\right) \\
& =\left(\sum_{a=1}^{r} m_{i a} \hbar \partial_{a}\right) \int_{\Gamma} e^{F_{q} / \hbar} \omega_{q} .
\end{aligned}
$$

By using this, we have

$$
\begin{aligned}
\mathcal{P}_{\boldsymbol{d}} \mathcal{I}(q, \hbar) & =\int_{\Gamma}\left(q^{\boldsymbol{d}} \prod_{\left\langle w_{i}, \boldsymbol{d}\right\rangle<0} \prod_{k=0}^{-\left\langle w_{i}, \boldsymbol{d}\right\rangle-1}\left(\hbar \mathrm{w}_{i} \frac{\partial}{\partial \mathrm{w}_{i}}-k \hbar\right)-\prod_{\left\langle w_{i}, \boldsymbol{d}\right\rangle>0} \prod_{k=0}^{\left\langle w_{i}, \boldsymbol{d}\right\rangle-1}\left(\hbar \mathrm{w}_{i} \frac{\partial}{\partial \mathrm{w}_{i}}-k \hbar\right)\right) e^{F_{q} / \hbar} \omega_{q} \\
& =\int_{\Gamma}\left(q^{\boldsymbol{d}} \prod_{\left\langle w_{i}, \boldsymbol{d}\right\rangle<0} \mathrm{w}_{i}^{-\left\langle w_{i}, \boldsymbol{d}\right\rangle}-\prod_{\left\langle w_{i}, \boldsymbol{d}\right\rangle>0} \mathrm{w}_{i}^{\left\langle w_{i}, \boldsymbol{d}\right\rangle}\right) e^{F_{q} / \hbar} \omega_{q}=0,
\end{aligned}
$$

where we used $q^{\boldsymbol{d}}=\prod_{i} \mathrm{w}_{i}^{\left\langle w_{i}, \boldsymbol{d}\right\rangle}$.

We call the system of hypergeometric differential equations $\left\{\mathcal{P}_{\boldsymbol{d}} \mathcal{I}(q, \hbar)=0 \mid \boldsymbol{d} \in \mathbb{Z}^{r}\right\}$ the Mellin system. Let $M_{\text {Mell }}$ be the $D$-module corresponding to the Mellin system

$$
M_{\text {Mell }}=\mathbb{C}\left\langle q^{ \pm}, \hbar \partial, \hbar\right\rangle / I_{\text {Mell }}, \quad I_{\text {Mell }}=\sum_{\boldsymbol{d} \in \mathbb{Z}^{r}} \mathbb{C}\left\langle q^{ \pm}, \hbar \partial, \hbar\right\rangle \mathcal{P}_{\boldsymbol{d}}
$$

where $q^{ \pm}$and $\hbar \partial$ are shorthand for $q_{1}^{ \pm}, \ldots, q_{r}^{ \pm}$and $\hbar q_{1} \partial / \partial q_{1}, \ldots, \hbar q_{r} \partial / \partial q_{r}$. The oscillatory integral $\mathcal{I}_{\Gamma}(q, \hbar)$ gives a solution of $M_{\text {Mell }}$ for each non-compact cycle $\Gamma$.

5.2. $S^{1}$-equivariant Floer cohomology. We review the algebraic construction of the equivariant Floer cohomology $F H_{S^{1}}^{*}$ for a toric variety $X$ briefly (see [16 for detail). For each $\boldsymbol{d} \in \mathbb{Z}^{r}$, we put $H_{S^{1}}^{*}\left(L_{\boldsymbol{d}}^{\infty}\right)=\mathbb{C}\left[P_{1}, \ldots, P_{r}, \hbar\right]$. This is an algebra over $H_{S^{1}}^{*}(\mathrm{pt})=\mathbb{C}[\hbar]$. When $\left\langle w_{i}, \boldsymbol{d}\right\rangle \geq\left\langle w_{i}, \boldsymbol{d}^{\prime}\right\rangle$ for all $i$, we define a push-forward map $i_{\boldsymbol{d}, \boldsymbol{d}^{\prime}}: H_{S^{1}}^{*}\left(L_{\boldsymbol{d}}^{\infty}\right) \rightarrow H_{S^{1}}^{*}\left(L_{\boldsymbol{d}^{\prime}}^{\infty}\right)$ by $i_{\boldsymbol{d}, \boldsymbol{d}^{\prime}}(\alpha)=\alpha \prod_{i=1}^{N} \prod_{k=\left\langle w_{i}, \boldsymbol{d}^{\prime}\right\rangle}^{\left\langle w_{i}, \boldsymbol{d}\right\rangle-1} W_{i, k}$, where $W_{i, k}=\sum_{a=1}^{r} m_{i a} P_{a}-k \hbar$. Then, we have an inductive system $\left(H_{S^{1}}^{*}\left(L_{\boldsymbol{d}}^{\infty}\right), i_{\boldsymbol{d}, \boldsymbol{d}^{\prime}}\right)$. Let $H_{S^{1}}^{\infty / 2}=\lim _{\boldsymbol{d}} H_{S^{1}}^{*}\left(L_{\boldsymbol{d}}^{\infty}\right)$ be its direct limit. We define an $H_{S^{1}}^{*}(\mathrm{pt})$ algebra homomorphism $Q^{\boldsymbol{d}}=\prod_{a=1}^{r} Q_{a}^{d_{a}}: H_{S^{1}}^{*}\left(L_{\boldsymbol{d}^{\prime}}^{\infty}\right) \rightarrow H_{S^{1}}^{*}\left(L_{\boldsymbol{d}^{\prime}+\boldsymbol{d}}^{\infty}\right)$ by $Q^{\boldsymbol{d}}\left(P_{a}\right)=P_{a}-d_{a} \hbar$. This is compatible with the direct limit and we have a module homomorphism $Q^{d}: H_{S^{1}}^{\infty / 2} \rightarrow H_{S^{1}}^{\infty / 2}$. We can check that the multiplication by $P_{a}$ and the action of $Q_{b}$ on $H_{S^{1}}^{\infty / 2}$ satisfies the commutation relation $\left[P_{a}, Q_{b}\right]=\hbar \delta_{a b} Q_{b}$. Hence, $H_{S^{1}}^{\infty / 2}$ has a $D$-module structure when we regard $P_{a}$ as a differential operator $\hbar Q_{a}\left(\partial / \partial Q_{a}\right)$. Let $\Delta_{\boldsymbol{d}} \in H_{S^{1}}^{\infty / 2}$ be the image of $1 \in H_{S^{1}}^{*}\left(L_{\boldsymbol{d}}^{\infty}\right)$. We 
also write $\Delta=\Delta_{0}$. Let $F H_{0}$ be the submodule $\mathbb{C}\left\langle P_{1}, \ldots, P_{r}, Q_{1}, \ldots, Q_{r}, \hbar\right\rangle \Delta$ of $H_{S^{1}}^{\infty / 2}$. This $F H_{0}$ has a natural $Q$-adic topology and we define $F H_{S^{1}}^{*}$ as the completion of $F H_{0}$ :

$$
F H_{S^{1}}^{*}=\widehat{F H_{0}}={\underset{n}{n}}_{\lim _{n}} F H_{0} / \mathfrak{m}^{n} F H_{0},
$$

where $\mathfrak{m}=\sum_{a} Q_{a} \mathbb{C}[Q, \hbar]$. This becomes a module over $\mathbb{C}[\hbar] \llbracket Q_{1}, \ldots, Q_{r} \rrbracket\left\langle P_{1}, \ldots, P_{r}\right\rangle$.

Proposition 5.2. The D-module $H_{S^{1}}^{\infty}$ is generated by $\Delta$ as a $\mathbb{C}\left\langle Q^{ \pm}, P, \hbar\right\rangle$-module and all the relations are generated by $\widetilde{\mathcal{P}}_{\boldsymbol{d}}=Q^{\boldsymbol{d}} \prod_{\left\langle w_{i}, \boldsymbol{d}\right\rangle<0} \prod_{k=0}^{-\left\langle w_{i}, \boldsymbol{d}\right\rangle-1} W_{i, k}-\prod_{\left\langle w_{i}, \boldsymbol{d}\right\rangle>0} \prod_{k=0}^{\left\langle w_{i}, \boldsymbol{d}\right\rangle-1} W_{i, k}$ for $\boldsymbol{d} \in \mathbb{Z}^{r}$. Therefore, $H_{S^{1}}^{\infty / 2}$ is isomorphic to $M_{\text {Mell }}$.

Proof. By the relation $\Delta_{\boldsymbol{d}}=Q^{\boldsymbol{d}} \Delta, H_{S^{1}}^{\infty / 2}$ is generated by $\Delta$. It is easy to check that $\widetilde{\mathcal{P}}_{\boldsymbol{d}} \Delta=0$. Assume that $f(P, Q, \hbar) \Delta=0$. We set $f(P, Q, \hbar)=\sum_{i} f_{i}(P, \hbar) Q^{\boldsymbol{d}_{i}}$. There exists $\boldsymbol{d}$ such that $\left\langle w_{j}, \boldsymbol{d}_{i}+\boldsymbol{d}\right\rangle>0$ for all $i, j$. Then we have

$$
\begin{aligned}
Q^{\boldsymbol{d}} f(P, Q, \hbar) & =\sum_{i} f_{i}\left(P_{a}-\left\langle p_{a}, \boldsymbol{d}\right\rangle \hbar, \hbar\right) Q^{\boldsymbol{d}_{i}+\boldsymbol{d}} \\
& =\sum_{i} f_{i}\left(P_{a}-\left\langle p_{a}, \boldsymbol{d}\right\rangle \hbar, \hbar\right)\left(\widetilde{\mathcal{P}}_{\boldsymbol{d}_{i}+\boldsymbol{d}}-\prod_{j} \prod_{k=0}^{\left\langle w_{j}, \boldsymbol{d}_{i}+\boldsymbol{d}\right\rangle-1} W_{j, k}\right) .
\end{aligned}
$$

When applying this to $\Delta$, we have $\sum_{i} f_{i}\left(P_{a}-\left\langle p_{a}, \boldsymbol{d}\right\rangle \hbar, \hbar\right) \prod_{j} \prod_{k=0}^{\left\langle w_{j}, \boldsymbol{d}_{i}+\boldsymbol{d}\right\rangle-1} W_{j, k} \Delta=0$. Because the canonical map $H_{S^{1}}^{*}\left(L_{0}^{\infty}\right) \rightarrow H_{S^{1}}^{\infty}$ is injective, we have

$$
\sum_{i} f_{i}\left(P_{a}-\left\langle p_{a}, \boldsymbol{d}\right\rangle \hbar, \hbar\right) \prod_{j} \prod_{k=0}^{\left\langle w_{j}, \boldsymbol{d}_{i}+\boldsymbol{d}\right\rangle-1} W_{j, k}=0 .
$$

Therefore, by (25), we have $f(P, Q, \hbar)=\sum_{i} f_{i}(P, \hbar) Q^{-\boldsymbol{d}} \widetilde{\mathcal{P}}_{\boldsymbol{d}_{i}+\boldsymbol{d}}$.

Corollary 5.3. Under the correspondence $q_{a} \mapsto Q_{a}, \hbar \partial_{a} \mapsto P_{a}$, we have

$$
F H_{0} \cong \mathbb{C}\langle q, \hbar \partial, \hbar\rangle / I_{\text {poly }}, \quad I_{\text {poly }}=I_{\text {Mell }} \cap \mathbb{C}\langle q, \hbar \partial, \hbar\rangle .
$$

Hereafter, we identify $Q_{a}$ and $P_{a}$ with $q_{a}$ and $\hbar \partial_{a}$. From this, we can describe $F H_{S^{1}}^{*}$ as follows.

\section{Proposition 5.4.}

$$
F H_{S^{1}}^{*}=\widehat{F H}_{0} \cong \mathbb{C}[\hbar] \llbracket q \rrbracket\langle\hbar \partial\rangle / \bar{I}_{\text {poly }},
$$

where $\bar{I}_{\text {poly }} \subset \mathbb{C}[\hbar] \llbracket q \rrbracket\langle\hbar \partial\rangle$ is the closure of $I_{\text {poly }}$ with respect to the q-adic topology.

Proof. In [16], section 4.4, it is proved that $F H_{S^{1}}^{*}$ is generated by $\Delta$ over $\mathbb{C}[\hbar] \llbracket Q \rrbracket\langle P\rangle$. Therefore we have a surjection $\mathbb{C}[\hbar] \llbracket Q \rrbracket\langle P\rangle \rightarrow F H_{S^{1}}^{*}$. Assume $f(P, Q, \hbar)=\sum_{\boldsymbol{d} \geq 0} f_{\boldsymbol{d}}(P, \hbar) Q^{\boldsymbol{d}} \in$ $\mathbb{C}[\hbar] \llbracket Q \rrbracket\langle P\rangle$ satisfies $f(P, Q, \hbar) \Delta=0$. Set $f_{n}=\sum_{|\boldsymbol{d}| \leq n} f_{\boldsymbol{d}}(P, \hbar) Q^{\boldsymbol{d}}$. Then,

$$
f_{n} \Delta=-\sum_{|\boldsymbol{d}|>n} f_{\boldsymbol{d}}(P, \hbar) Q^{\boldsymbol{d}} \Delta
$$

belongs to $F H_{0} \cap \mathfrak{m}^{n+1} \widehat{F H}_{0}=\mathfrak{m}^{n+1} F H_{0}$. Therefore, there exists $g_{n}$ in $\mathfrak{m}^{n+1} \mathbb{C}\langle Q, P, \hbar\rangle$ such that $f_{n} \Delta=g_{n} \Delta$. Because $f_{n}-g_{n} \in I_{\text {poly }}$ and $f_{n}-g_{n} \rightarrow f$ as $n \rightarrow \infty$, we have $f \in \bar{I}_{\text {poly }}$. 
Later, we will see that $\bar{I}_{\text {poly }}$ does not necessarily coincide with $\mathbb{C}[\hbar] \llbracket q \rrbracket\langle\hbar \partial\rangle I_{\text {poly }}$. In such a case, we need to add non-algebraic differential equations. We set $\mathcal{O}_{\text {small }}^{\hbar}=\mathcal{O}_{\lambda}^{\hbar} \cap \mathbb{C}[\hbar] \llbracket q \rrbracket\left(\mathcal{O}_{\lambda}^{\hbar}\right.$ was introduced in section 4):

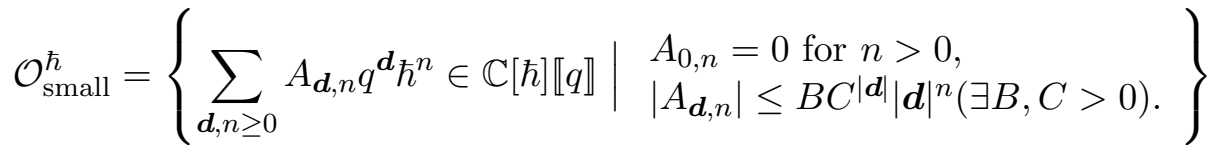

Let $\widetilde{F H}_{S^{1}}^{*}$ be the following submodule of $F H_{S^{1}}^{*}$ :

$$
\widetilde{F H}_{S^{1}}^{*}=\mathcal{O}_{\text {small }}^{\hbar}\left\langle P_{1}, \ldots, P_{r}\right\rangle \Delta \subset F H_{S^{1}}^{*} .
$$

Then we have $\widetilde{F H}_{S^{1}}^{*} \cong \mathcal{O}_{\text {small }}^{\hbar}\langle\hbar \partial\rangle / \bar{I}_{\text {poly }}$, where $\bar{I}_{\text {poly }}$ is the closure of $I_{\text {poly }}$ in $\mathcal{O}_{\text {small }}^{\hbar}\langle\hbar \partial\rangle$ with respect to the $q$-adic topology. Then we have a surjection

$$
M_{\text {Mell }} \otimes_{\mathbb{C}\left[\hbar, q^{ \pm}\right]} \mathcal{O}_{\text {small }}^{\hbar}\left[q^{-1}\right]=\left(\mathcal{O}_{\text {small }}^{\hbar}\langle\hbar \partial\rangle / \mathcal{O}_{\text {small }}^{\hbar} I_{\text {poly }}\right)\left[q^{-1}\right] \longrightarrow \widetilde{F H}_{S^{1}}^{*}\left[q^{-1}\right]
$$

Proposition 5.5. Let $\left\{T_{i}\left(y_{1}, \ldots, y_{r}\right)\right\}_{i=0}^{s}$ be homogeneous polynomials such that $\left\{T_{i}\left(p_{1}, \ldots, p_{r}\right)\right\}_{i=0}^{s}$ forms a basis of $H^{*}(X)$. Then, $\left\{T_{i}(P) \Delta\right\}_{i=1}^{s}$ forms a free basis of $\widetilde{F H}_{S^{1}}^{*}$ as an $\mathcal{O}_{\text {small }}^{\hbar}$-module. Moreover, we have $F H_{S^{1}}^{*}=\widetilde{F H}_{S^{1}}^{*} \otimes_{\mathcal{O}_{\text {small }}^{\hbar}} \mathbb{C}[\hbar] \llbracket q \rrbracket$.

Proof. In [16], section 4.4, we showed that $\left\{T_{i}(P) \Delta\right\}_{i=0}^{s}$ forms a free basis of $F H_{S^{1}}^{*}$ as a $\mathbb{C}[\hbar] \llbracket q \rrbracket$ module. Here, we will show this is also a basis of $\widetilde{F H}_{S^{1}}^{*}$ as a $\mathcal{O}_{\text {small }}^{\hbar}$-module. It suffices to show that the connection matrix $\Omega_{a}=\left(\Omega_{a ; i j}\right)$ defined by

$$
P_{a} T_{j}(P) \Delta=\sum_{i=0}^{s} \Omega_{a ; i j}(q, \hbar) T_{i}(P) \Delta
$$

has entries in $\mathcal{O}_{\text {small }}^{\hbar}$. We use a method similar to the proof in section 4.1. By [16], section 4.3, there exists a map $\Xi: F H_{S^{1}}^{*} \rightarrow H^{*}(X) \otimes \mathbb{C}\left[\hbar, \hbar^{-1}\right] \llbracket q \rrbracket$ and a pairing $(\cdot, \cdot): F H_{*}^{S^{1}} \times F H_{S^{1}}^{*} \rightarrow$ $\mathbb{C}[\hbar] \llbracket q \rrbracket$ such that

$$
\Xi\left(P_{a} \alpha\right)=\left(\hbar \partial_{a}+p_{a}\right) \Xi(\alpha), \quad(\bar{\alpha}, \beta)=\int_{X} \overline{\Xi(\alpha)} \cup \Xi(\beta),
$$

for $\alpha, \beta \in F H_{S^{1}}^{*}$. Here, the equivariant Floer homology $F H_{*}^{S^{1}}$ is the Poincaré dual theory of $F H_{S^{1}}^{*}$ with a $\mathbb{C} \llbracket q \rrbracket$-module isomorphism ${ }^{-}: F H_{S^{1}}^{*} \cong F H_{*}^{S^{1}}$ such that $\overline{\hbar \alpha}=-\hbar \bar{\alpha}$. The operator - also acts on $H^{*}(X)\left[\hbar, \hbar^{-1}\right] \llbracket q \rrbracket$ by changing the sign of $\hbar$. The function $\widetilde{I}(q, \hbar)=\Xi(\Delta)$ is written as

$$
\widetilde{I}(q, \hbar)=\sum_{\boldsymbol{d} \geq 0} q^{\boldsymbol{d}} \prod_{i=1}^{r+N} \frac{\prod_{k=1+\left\langle w_{i}, \boldsymbol{d}\right\rangle}^{\infty}\left(w_{i}+k \hbar\right)}{\prod_{k=1}^{\infty}\left(w_{i}+k \hbar\right)} .
$$

Here, $I(q, \hbar)=e^{p \log q / \hbar} \widetilde{I}(q, \hbar)$ is frequently referred to as $I$-function. We write $\widetilde{I}(q, \hbar)=$ $\sum_{\boldsymbol{d} \geq 0} I_{\boldsymbol{d}}(\hbar) q^{\boldsymbol{d}}$. Take $C_{1}>0$ such that $\sup _{|\hbar|=1, k \geq 1, i}\left(\left\|w_{i}\right\|,\left\|1+w_{i} /(k \hbar)\right\|,\left\|\left(1+w_{i} /(k \hbar)\right)^{-1}\right\|\right) \leq$ $C_{1}$. Then we have for $|\hbar|=1$

$$
\begin{aligned}
\left\|I_{\boldsymbol{d}}(\hbar)\right\| & \leq \frac{\prod_{\left\langle w_{i}, \boldsymbol{d}\right\rangle<0}\left|\left\langle w_{i}, \boldsymbol{d}\right\rangle\right| !}{\prod_{\left\langle w_{i}, \boldsymbol{d}\right\rangle>0}\left\langle w_{i}, \boldsymbol{d}\right\rangle !} C_{1}^{\sum_{i=1}^{r+N}\left|\left\langle w_{i}, \boldsymbol{d}\right\rangle\right|} \\
& \leq C_{2}^{|\boldsymbol{d}|} \begin{cases}1 /\left\langle c_{1}(X), \boldsymbol{d}\right\rangle ! & \text { if }\left\langle c_{1}(X), \boldsymbol{d}\right\rangle \geq 0, \\
\left|\left\langle c_{1}(X), \boldsymbol{d}\right\rangle\right| ! & \text { if }\left\langle c_{1}(X), \boldsymbol{d}\right\rangle \leq 0 .\end{cases}
\end{aligned}
$$


for some $C_{2}>0$. Here, we used $c_{1}(X)=\sum_{i=1}^{r+N} w_{i}$. Let $\mathcal{O}_{\text {deg }}^{\hbar, \hbar^{-1}}$ be the set of power series $\sum_{\boldsymbol{d}} A_{\boldsymbol{d}}(\hbar) q^{\boldsymbol{d}}$ in $\mathbb{C}\left[\hbar, \hbar^{-1}\right] \llbracket q \rrbracket$ satisfying

$$
\sup _{|\hbar|=1}\left|A_{\boldsymbol{d}}(\hbar)\right| \leq B C^{\boldsymbol{d}} \begin{cases}1 /\left\langle c_{1}(X), \boldsymbol{d}\right\rangle ! & \text { if }\left\langle c_{1}(X), \boldsymbol{d}\right\rangle \geq 0, \\ \left|\left\langle c_{1}(X), \boldsymbol{d}\right\rangle\right| ! & \text { if }\left\langle c_{1}(X), \boldsymbol{d}\right\rangle \leq 0,\end{cases}
$$

for some $B, C>0$. ( $B, C$ depend on each element.) As in section 4.1] we can prove that $\mathcal{O}_{\mathrm{deg}}^{\hbar, \hbar^{-1}}$ is a subring of $\mathbb{C}\left[\hbar, \hbar^{-1}\right] \llbracket q \rrbracket$. Each component of $\widetilde{I}(q, \hbar)$ belongs to the ring $\mathcal{O}_{\mathrm{deg}}^{\hbar, \hbar^{-1}}$. Set $\eta_{k j}=\left(\overline{T_{k}(P) \Delta}, T_{j}(P) \Delta\right)$. By using (27), we have

$$
\begin{aligned}
\eta_{k j} & =\int_{X} T_{k}(-\hbar \partial+p) \widetilde{I}(q,-\hbar) \cup T_{j}(\hbar \partial+p) \widetilde{I}(q, \hbar) \\
\sum_{i=0}^{s} \eta_{k i} \Omega_{a ; i j} & =\left(\overline{T_{k}(P) \Delta}, P_{a} T_{j}(P) \Delta\right) \\
& =\int_{X} T_{k}(-\hbar \partial+p) \widetilde{I}(q,-\hbar) \cup\left(\hbar \partial_{a}+p_{a}\right) T_{j}(\hbar \partial+p) \widetilde{I}(q, \hbar) .
\end{aligned}
$$

Therefore, $\eta_{k j}$ and $\sum_{i} \eta_{k i} \Omega_{a ; i j}$ are contained in $\mathcal{O}_{\mathrm{deg}}^{\hbar, \hbar^{-1}} \cap \mathbb{C}[\hbar] \llbracket q \rrbracket$. Moreover, these elements are homogeneous with respect to the grading $\operatorname{deg} q^{\boldsymbol{d}}=2\left\langle c_{1}(X), \boldsymbol{d}\right\rangle$ and $\operatorname{deg} \hbar=2$. The homogeneity means that the coefficient of $q^{\boldsymbol{d}} \hbar^{n}$ is non-vanishing only when $\left\langle c_{1}(X), \boldsymbol{d}\right\rangle+n$ equals a given constant. Therefore, by Cauchy's residue theorem, we can see that $\eta_{k j}$ and $\sum_{i} \eta_{k i} \Omega_{a ; i j}$ are also contained in $\mathcal{O}_{\text {small }}^{\hbar}$. Because $\lim _{q \rightarrow 0} \eta_{k j}=\int_{X} T_{k}(p) \cup T_{j}(p)$ is an invertible matrix and $\mathcal{O}_{\text {small }}^{\hbar}$ is a local ring, we have $\Omega_{a ; i j} \in \mathcal{O}_{\text {small }}^{\hbar}$.

5.3. Generalized mirror transformation. We can describe how to reconstruct the quantum $D$-module $Q D M^{*}(X)$ from $F H_{S^{1}}^{*}$ as follows:

Theorem 5.6 (19], [16] Theorem 4.9, 5.4). There exists a formal embedding $\mathfrak{e m b}:\left(\mathbb{C}^{r}, 0\right) \rightarrow$ $\left(\mathbb{C}^{s+1}, 0\right)$ and an isomorphism of D-modules $\Phi_{\mathfrak{e m b}}: \mathfrak{e m b}^{*}(Q D M(X)) \cong F H_{S^{1}}^{*}$. The map $\mathfrak{e m b}$ is given by equations of the form

$$
\hat{t}_{0}=F_{0}(q), \hat{q}_{1}=q_{1} \exp \left(F_{1}(q)\right), \ldots, \hat{q}_{r}=q_{r} \exp \left(F_{r}(q)\right), \hat{t}_{r+1}=F_{r+1}(q), \ldots, \hat{t}_{s}=F_{s}(q)
$$

for some $F_{i}(q) \in \mathbb{C} \llbracket q \rrbracket, F_{i}(0)=0$ and $\left.\Phi_{\mathfrak{e m b}}\right|_{q=0}$ is determined by the canonical isomorphism $H^{*}(X) \otimes \mathbb{C}[\hbar] \cong F H_{S^{1}}^{*} / \sum_{a=1}^{r} q_{a} F H_{S^{1}}^{*}$. Moreover, we can reconstruct $Q D M^{*}(X)$ from $F H_{S^{1}}^{*}$ by the following steps:

(i) Take a free basis $\left\{T_{0}, \ldots, T_{s}\right\}$ of $F H_{S^{1}}^{*}$ as a $\mathbb{C}[\hbar] \llbracket q \rrbracket$-module as in Proposition 5.5 and calculate a connection matrix $\Omega_{a}$ defined by $P_{a} T_{j}=\sum_{i=0}^{s} \Omega_{a ; i j} T_{i}$.

(ii) Find a gauge transformation $g$ such that $\left.g\right|_{q=0}=$ id and that the new connection matrix $\widehat{\Omega}_{a}$ is $\hbar$-independent, where $\widehat{\Omega}_{a}=g^{-1} \Omega_{a} g+g^{-1} \hbar \partial_{a} g$.

(iii) Solve for matrix-valued functions $\widehat{\Omega}_{j}\left(t_{0}, q, t_{r+1}, \ldots, t_{s}\right)(0 \leq j \leq s)$ from $\widehat{\Omega}_{a}(q)(1 \leq a \leq$ $r)$, where $\widehat{\Omega}_{0}=\mathrm{id}$. This procedure will be reviewed in Proposition 5.8 .

(iv) Find a new coordinate system $\left(\hat{t}_{0}, \hat{q}_{1}, \ldots, \hat{q}_{r}, \hat{t}_{r+1}, \ldots, \hat{t}_{s}\right)$ of the form $\hat{t}_{0}=t_{0}+F_{0}(q)$, $\hat{q}_{a}=q_{a} \exp \left(F_{a}(q)\right)(1 \leq a \leq r), \hat{t}_{j}=t_{j}+F_{j}(q)(r+1 \leq j \leq s)$ such that $\widehat{\Omega}_{\hat{j}}=\sum_{i=0}^{s}\left(\partial t_{i} / \partial \hat{t}_{j}\right) \widehat{\Omega}_{i}$ satisfies $\widehat{\Omega}_{\hat{j}}(1)=p_{j}$.

We study these four steps from an analytic point of view. By Proposition 5.5, the connection matrix $\Omega_{a}(q)$ in step (i) has its matrix elements in $\mathcal{O}_{\text {small }}^{\hbar}$. Then by Proposition 4.8, the connection matrix $\widehat{\Omega}_{a}$ in step (ii) becomes a convergent function of $q$. In Proposition [5.8, we will see that the reconstruction step (iii) preserves the convergence. The last step (iv) can 
be done in the convergent category, therefore $Q H^{*}(X)$ has convergent structure constants. Summarizing,

Theorem 5.7. The quantum $D$-module $Q D M^{*}(X)$ of a toric variety is defined over convergent power series. The embedding $\mathfrak{e m b}:\left(\mathbb{C}^{r}, 0\right) \rightarrow\left(\mathbb{C}^{s+1}, 0\right)$ in Theorem $[5.6$ is complex analytic and $\widetilde{F H}_{S^{1}}^{*} \cong \mathfrak{e m b}^{*}\left(Q D M_{\mathrm{an}}^{*}(X)\right) \otimes_{\mathcal{O}_{\mathrm{an}}^{\hbar}} \mathcal{O}_{\text {small }}^{\hbar}$, where $Q D M_{\mathrm{an}}^{*}(X)=\left(H^{*}(X) \otimes \mathcal{O}_{\mathrm{an}}^{\hbar}, \nabla^{\hbar}\right)$ and $\mathcal{O}_{\mathrm{an}}^{\hbar}=\left\{f(\hbar, x) \in \mathbb{C}\{\hbar, x\}|f|_{q=0}\right.$ is constant. $\}$.

In order to state the compatibility of reconstruction with convergence, we consider the following general situation. Consider a flat connection $\nabla^{\hbar}=\hbar d+\sum_{a=1}^{r} \Omega_{a}(q) d q_{a} / q_{a}$ of the bundle $H^{*}(X) \times U \rightarrow U$ regular singular along $q_{1} \cdots q_{r}=0$. Here, $U$ is a neighborhood of 0 in $\mathbb{C}^{r}$ and $\Omega_{a}(q)$ is an $\hbar$-independent holomorphic function on $U$.

Proposition 5.8. Assume that $H^{*}(X)$ is generated by 1 under the action of residue matrices $p_{a}:=\Omega_{a}(0)$. For a set of vectors $v_{1}, \ldots, v_{l}$ in $H^{*}(X), \nabla^{\hbar}$ can be uniquely extended to a flat connection $\widetilde{\nabla}^{\hbar}=\hbar d+\sum_{a=1}^{r} \Omega_{a}(q, t) d q_{a} / q_{a}+\sum_{j=1}^{l} \Phi_{j}(q, t) d t_{j}$ defined on a neighborhood $U^{\prime}$ of 0 in $\mathbb{C}^{r+l}$ under the condition that $\Phi_{j}(q, t) 1=v_{j}$.

Proof. In [17, Theorem 4.9, we proved that $\widetilde{\nabla}^{\hbar}$ can be reconstructed uniquely as a formal connection. Here, we prove that this is convergent. Because we can extend $\nabla^{\hbar}$ to the $t_{j^{-}}$ direction independently for different $t_{j}$ 's, we can assume that $l=1$. Flatness of the connection $\widetilde{\nabla}^{\hbar}$ implies $\partial_{t} \Omega_{a}=q_{a} \partial_{q_{a}} \Phi$ and $\left[\Omega_{a}, \Phi\right]=0$. By taking a smaller $U$ if necessary, we can assume that $H^{*}(X)$ is generated by 1 under the action of $\Omega_{a}(q)$ for each $q \in U$. Then, we have a surjection $\mathbb{C}\left[\Omega_{1}(q), \ldots, \Omega_{r}(q)\right] \rightarrow H^{*}(X)$ and this determines a product $*_{q}$ on $H^{*}(X)$ for each $q \in U$. On the locus $\{t=0\}, \Phi(q, 0)$ must be a multiplication $\left(v_{1} *_{q}\right)$ by $v_{1}$ because it commutes with $\Omega_{a}(q)$. Therefore, $\Phi(q, 0)$ becomes holomorphic on $U$. On the locus $\{q=0\}$, it is easy to see that $\Omega_{a}(0, t)=p_{a}$ and $\Phi(0, t)=v_{1} \cup$. Expand $\Phi$ and $\Omega_{a}$ as $\Phi=\sum_{\boldsymbol{d}, m \geq 0} \Phi_{\boldsymbol{d}, m} q^{\boldsymbol{d}_{t}}$ and $\Omega_{a}=\sum_{\boldsymbol{d}, m>0} \Omega_{a ; \boldsymbol{d}, m} q^{\boldsymbol{d}^{m}}{ }^{m}$. Note that $\Phi_{0, m}=\delta_{0 m}\left(v_{1} \cup\right)$ and $\Omega_{a ; 0, m}=\delta_{0 m} p_{a}$. Then by $\partial_{t} \Omega_{a}=q_{a} \partial_{q_{a}} \Phi$, we have

$$
\Omega_{a ; \boldsymbol{d}, m+1}=\frac{d_{a}}{m+1} \Phi_{\boldsymbol{d}, m}
$$

Let $\left\{T_{0}, \ldots, T_{s}\right\}$ be a basis of $H^{*}(X)$. By the assumption, we can write

$$
T_{i}=\sum_{l, a_{1}, \ldots, a_{l}} A_{a_{1}, \ldots, a_{l}}^{(i)} p_{a_{1}} \cdots p_{a_{l}}(1)
$$

for some $A_{a_{1}, \ldots, a_{l}}^{i} \in \mathbb{C}$. Then by $\Phi_{\boldsymbol{d}, m+1}(1)=0$, we have

$$
\begin{aligned}
\Phi_{\boldsymbol{d}, m+1} T_{i} & =\sum_{l, a_{1}, \ldots, a_{l}} A_{a_{1}, \ldots, a_{l}}^{(i)} \sum_{k=1}^{l} p_{a_{1}} \cdots\left[\Phi_{\boldsymbol{d}, m+1}, p_{a_{k}}\right] \cdots p_{a_{l}}(1) \\
& =\sum_{l, a_{1}, \ldots, a_{l}} A_{a_{1}, \ldots, a_{l}}^{(i)} \sum_{k=1}^{l} p_{a_{1}} \cdots\left(\sum_{\boldsymbol{d}_{1}>0} \sum_{j=0}^{m+1}\left[\Omega_{a_{k} ; \boldsymbol{d}_{1}, j}, \Phi_{\boldsymbol{d}-\boldsymbol{d}_{1}, m+1-j}\right]\right) \cdots p_{a_{l}}(1)
\end{aligned}
$$

where we used $\left[\Phi, \Omega_{a_{k}}\right]=0$. Assume by induction that we know $\Omega_{a ; \boldsymbol{d}^{\prime}, m}$ and $\Phi_{\boldsymbol{d}^{\prime}, m}$ for all $\left|\boldsymbol{d}^{\prime}\right|<\bar{d}$ and all $m \geq 0$. Set $\omega_{d, m}=\max _{a} \sum_{|\boldsymbol{d}|=d}\left\|\Omega_{a ; \boldsymbol{d}, m}\right\|$ and $\phi_{d, m}=\sum_{|\boldsymbol{d}|=d}\left\|\Phi_{\boldsymbol{d}, m}\right\|$. Assume also that there exist constants $A, C, M>1$ such that

$$
\omega_{d, m} \leq A \frac{C^{d+m}}{(d+1)^{M}} \frac{d^{m}}{m !}, \quad \phi_{d, m} \leq A \frac{C^{d+m}}{(d+1)^{M}} \frac{d^{m}}{m !}
$$


holds for all $d<\bar{d}$ and all $m \geq 0$. We must choose $A, C, M$ so that this estimate is valid for $m=0$ and all $d \geq 0$. Take $\boldsymbol{d}$ such that $|\boldsymbol{d}|=\bar{d}$. First we can solve for $\Omega_{a ; \boldsymbol{d}, 1}$ by (29). Then we solve for $\Phi_{\boldsymbol{d}, 1}$ by (30). Next we solve for $\Omega_{a ; \boldsymbol{d}, 2}$ by (29) and repeat this process. Assume that the estimate (31) holds for $d=\bar{d}$ and up to $m(m \geq 0)$. We have by (29),

$$
\omega_{\bar{d}, m+1} \leq \frac{\bar{d}}{m+1} \phi_{\bar{d}, m} \leq A \frac{C^{\bar{d}+m}}{(\bar{d}+1)^{M}} \frac{\bar{d}^{m+1}}{(m+1) !} .
$$

Next we have by (30),

$$
\begin{aligned}
\phi_{\bar{d}, m+1} & \leq B_{1} \sum_{0<i<\bar{d}} \sum_{j=0}^{m+1} \omega_{i, j} \phi_{\bar{d}-i, m+1-j}+B_{1} \omega_{\bar{d}, m+1}\left\|\left(v_{1} \cup\right)\right\| \\
& \leq B_{1} A^{2} \frac{C^{\bar{d}+m+1}}{(\bar{d}+1)^{M}} \frac{\bar{d}^{m+1}}{(m+1) !} \sum_{i=1}^{\bar{d}-1} \frac{(\bar{d}+1)^{M}}{(i+1)^{M}(\bar{d}-i+1)^{M}}+B_{2} A \frac{C^{\bar{d}+m}}{(\bar{d}+1)^{M}} \frac{\bar{d}^{m+1}}{(m+1) !} \\
& \leq A \frac{C^{\bar{d}+m+1}}{(\bar{d}+1)^{M}} \frac{\bar{d}^{m+1}}{(m+1) !}\left\{B_{1} A \varepsilon_{1}(M)+B_{2} / C\right\}
\end{aligned}
$$

where $B_{1}, B_{2}>0$ are constants determined only by $\left(H^{*}(X), \cup\right)$ and $\varepsilon_{1}(M)$ is a function defined in the proof of Proposition 4.8. To complete the induction, we need to choose $C$ and $M$ carefully. Because $\lim _{M \rightarrow \infty} \varepsilon_{1}(M)=0$, for sufficiently large $M$, we have $B_{1} A \varepsilon_{1}(M) \leq 1 / 2$. Next we choose $C$ sufficiently large so that the estimate (31) is valid for $m=0$ and all $d \geq 0$ and that $C \geq 2 B_{2}$. Then by (32), we have the desired estimate for $\phi_{\bar{d}, m+1}$. After we obtain the estimate (31) for all $d, m \geq 0$, we can see that $\Omega_{a}(q, t)$ and $\Phi(q, t)$ are convergent because

$$
\sum_{d \geq 0} \sum_{m \geq 0} C^{d+m} \frac{d^{m}}{m !} x^{d} y^{m}=\frac{1}{1-C x \exp (C y)}
$$

is holomorphic around $(x, y)=(0,0)$.

When we apply this proposition to the dual Givental connections, we have

Corollary 5.9. Let $X$ be a smooth projective variety. If $H^{*}(X)$ is generated by $H^{2}(X)$ and if the small quantum cohomology of $X$ has convergent structure constants, so does the big quantum cohomology $Q H^{*}(X)$.

5.4. Characteristic variety and semisimplicity. We study the characteristic variety of the Mellin system and proves the semisimplicity. For a differential operator $f(q, \hbar \partial, \hbar)$, we define its principal symbol as $\sigma(f)=f(q, \mathrm{p}, 0)$. Here, $\mathrm{p}_{1}, \ldots, \mathrm{p}_{r}$ are conjugate variables. We also define

$$
\begin{aligned}
\sigma\left(M_{\text {Mell }}\right) & =\mathbb{C}\left[q^{ \pm}, \mathrm{p}\right] / \sigma\left(I_{\text {Mell }}\right) \\
\sigma\left(\widetilde{F H}_{S^{1}}^{*}\right) & =\mathbb{C}\{q\}[\mathrm{p}] \otimes_{\sigma} \widetilde{F H}_{S^{1}}^{*} \cong \mathbb{C}\{q\}[\mathrm{p}] / \sigma\left(\bar{I}_{\text {poly }}\right) .
\end{aligned}
$$

The characteristic varieties of $\mathrm{Ch}\left(M_{\text {Mell }}\right)$ and $\mathrm{Ch}\left(\widetilde{F H}_{S^{1}}^{*}\right)$ are defined as analytic spectra of $\sigma\left(M_{\text {Mell }}\right)$ and $\sigma\left(\widetilde{F H}_{S^{1}}^{*}\right)$ respectively. They are (germs of) analytic subvarieties of $\left(\mathbb{C}^{*}\right)^{r} \times \mathbb{C}^{r}=$ $T^{*}\left(\mathbb{C}^{*}\right)^{r}$. By the surjection (26), we have an embedding

$$
\left.\mathrm{Ch}\left(\widetilde{F H}_{S^{1}}^{*}\right)\right|_{q_{1} \cdots q_{r} \neq 0} \longrightarrow \mathrm{Ch}\left(M_{\mathrm{Mell}}\right)
$$

The characteristic variety of $M_{\text {Mell }}$ is the zero set of $\sigma\left(\mathcal{P}_{\boldsymbol{d}}\right)$ for all $\boldsymbol{d}$. Because there exists $\boldsymbol{d}$ such that $\left\langle w_{i}, \boldsymbol{d}\right\rangle>0$ for all $i$, we can see from $\sigma\left(\mathcal{P}_{\boldsymbol{d}}\right)=0$ that $\sum_{b=1}^{r} m_{i b} \mathrm{p}_{b} \neq 0$ if $q \in\left(\mathbb{C}^{*}\right)^{r}$. 
(Here, we need the compactness of $X$.) Thus,

$$
\operatorname{Ch}\left(M_{\text {Mell }}\right)=\left\{(q, \mathrm{p}) \in\left(\mathbb{C}^{*}\right)^{r} \times \mathbb{C}^{r} \mid \sum_{b=1}^{r} m_{i b} \mathrm{p}_{b} \neq 0, q_{a}=\prod_{i=1}^{r+N}\left(\sum_{b=1}^{r} m_{i b} \mathrm{p}_{b}\right)^{m_{i a}}\right\} .
$$

Let $\operatorname{Crit}\left(F_{q}\right)$ be the set of critical points of $F_{q}$ in $Y_{q}$. This forms a family over $\left(\mathbb{C}^{*}\right)^{r}$. For $x \in \operatorname{Crit}\left(F_{q}\right)$, the cotangent vector $d_{x} F$ in $T_{x}^{*} Y$ equals $\pi^{*}\left(\sum_{a=1}^{r} \mathrm{p}_{a} d \log q_{a}\right)$ for some $\mathrm{p}_{a}$. Hence we have a map $d F: \bigcup_{q \in\left(\mathbb{C}^{*}\right)^{r}} \operatorname{Crit}\left(F_{q}\right) \rightarrow T^{*}\left(\mathbb{C}^{*}\right)^{r}, x \mapsto \sum_{a=1}^{r} \mathrm{p}_{a} d \log q_{a}$.

Lemma 5.10. $d F: \bigcup_{q \in\left(\mathbb{C}^{*}\right)^{r}} \operatorname{Crit}\left(F_{q}\right) \cong \operatorname{Ch}\left(M_{\text {Mell }}\right) \subset T^{*}\left(\mathbb{C}^{*}\right)^{r}$.

Proof. By $d F=\pi^{*}\left(\sum_{a=1}^{r} \mathrm{p}_{a} d \log q_{a}\right)$ on $\bigcup_{q} \operatorname{Crit}\left(F_{q}\right)$, we see that $\mathrm{w}_{i}=\sum_{a=1}^{r} m_{i a} \mathrm{p}_{a}$ and so $(q, \mathrm{p}) \in \mathrm{Ch}\left(M_{\text {Mell }}\right)$. The inverse is given by $\mathrm{w}_{i}=\sum_{a=1}^{r} m_{i a} \mathrm{p}_{a}$.

Proposition 5.11. (i) The projection $\mathrm{Ch}\left(M_{\text {Mell }}\right) \rightarrow\left(\mathbb{C}^{*}\right)^{r}$ is a ramified covering of degree $N ! \operatorname{Vol}(\operatorname{Conv}(\Sigma))$, where $\operatorname{Conv}(\Sigma)$ is the convex hull of all the primitive generators $\vec{x}_{1}, \ldots, \vec{x}_{r+N}$ of 1-dimensional cones in the fan $\Sigma$ defining $X$.

(ii) There exist exactly $\operatorname{dim}_{\mathbb{C}} H^{*}(X)$ branches of the covering $\mathrm{Ch}\left(M_{\mathrm{Mell}}\right) \rightarrow\left(\mathbb{C}^{*}\right)^{r}$ corresponding to the subcovering $\left.\mathrm{Ch}\left(\widetilde{F H}_{S^{1}}^{*}\right)\right|_{q_{1} \cdots q_{r} \neq 0} \rightarrow\left(\mathbb{C}^{*}\right)^{r}$. These branches are characterized by the condition that $\mathrm{p}_{a} \rightarrow 0$ as $q \rightarrow 0$.

Proof. By Kushnirenko's theorem [23, the dimension of Jacobi ring $\mathbb{C}\left[s_{1}, \ldots, s_{N}\right] /\left\langle\partial_{s} F_{q}\right\rangle$ equals $N$ ! times the volume of the Newton polytope of $F_{q}=\sum_{i=1}^{r+N}\left(\prod_{a} q_{a}^{l_{i a}}\right) s^{\vec{x}_{i}}$. On the other hand, the projection $\pi: \operatorname{Ch}\left(M_{\text {Mell }}\right) \rightarrow\left(\mathbb{C}^{*}\right)^{r}$ is a submersion at generic p. (In fact, $d \pi=$ $\partial \log q_{a} / \partial \mathrm{p}_{b}=\sum_{i} m_{i a} \mathrm{w}_{i}^{-1} m_{i b}$ is positive definite when $\mathrm{w}_{i}>0$.) Hence we obtain (i) by Lemma 5.10, Each branch of $\mathrm{Ch}\left(\widetilde{F H}_{S^{1}}^{*}\right)$ corresponds to a branch of simultaneous eigenvalues $\left(\mathrm{p}_{1}, \ldots, \mathrm{p}_{r}\right)$ of the connection matrices $\left(\left.\Omega_{1}\right|_{\hbar=0}, \ldots,\left.\Omega_{r}\right|_{\hbar=0}\right)$. Because $\left.\Omega_{a}\right|_{q=0}$ is a nilpotent matrix (cup product by $p_{a}$ ), we see that $\mathrm{p}_{a} \rightarrow 0$ as $q \rightarrow 0$. On the other hand, we have $\left.\mathrm{Ch}\left(M_{\text {Mell }}\right) \cong \mathrm{Ch}\left(F H_{0}\right)\right|_{q_{1} \cdots q_{r} \neq 0}$ and the zero-fiber of $\mathrm{Ch}\left(F H_{0}\right)$ is the spectrum of $\sigma\left(F H_{0}\right) / \sum_{a=1}^{r} q_{a} \sigma\left(F H_{0}\right) \cong \sigma\left(F H_{0} / \mathfrak{m} F H_{0}\right) \cong \sigma\left(\widehat{F H}_{0} / \mathfrak{m} \widehat{F H}_{0}\right) \cong H^{*}(X)$. Therefore, there exist only $\operatorname{dim}_{\mathbb{C}} H^{*}(X)$ branches converging to $\mathrm{p}=0$ at $q=0$.

By Theorem [5.7 we see that $\mathfrak{e m b}^{*}\left(\mathrm{Ch}\left(Q D M_{\text {an }}^{*}(X)\right)\right) \cong \mathrm{Ch}\left(\widetilde{F H}_{S^{1}}^{*}\right)$. Therefore, the generic fiber of the characteristic variety of $Q D M_{\text {an }}^{*}(X)$ consists of $\operatorname{dim}_{\mathbb{C}} H^{*}(X)$ distinct points. This means that

Corollary 5.12. The quantum cohomology of a smooth, projective toric variety is generically semisimple. (See 24], Part I, section 3 for the definition of semisimplicity.)

As remarked in the introduction, this corollary together with Kawamata's result 21] shows that Bayer and Manin's modified Dubrovin's conjecture [1] holds for toric varieties.

The proposition below will clarify the role of the nef condition for $c_{1}(X)$.

Proposition 5.13. The following conditions are equivalent.

(i) $c_{1}(X)$ is nef.

(ii) $N$ ! $\operatorname{Vol}(\operatorname{Conv}(\Sigma))=\operatorname{dim}_{\mathbb{C}} H^{*}(X)$.

(iii) $\left.\mathrm{Ch}\left(\widetilde{F H}_{S^{1}}^{*}\right)\right|_{q_{1} \cdots q_{r} \neq 0} \cong \mathrm{Ch}\left(M_{\text {Mell }}\right)$ on a small neighborhood of $q=0$.

(iv) $\widetilde{F H}_{S^{1}}^{*} \cong F H_{0} \otimes_{\mathbb{C}[\hbar, q]} \mathcal{O}_{\text {small }}^{\hbar}\left(\cong \mathcal{O}_{\text {small }}^{\hbar}\langle\hbar \partial\rangle / \mathcal{O}_{\text {small }}^{\hbar} I_{\text {poly }}\right)$.

(v) $F H_{S^{1}}^{*} \cong F H_{0} \otimes_{\mathbb{C}[\hbar, q]} \mathbb{C}[\hbar] \llbracket q \rrbracket\left(\cong \mathbb{C}[\hbar] \llbracket q \rrbracket\langle\hbar \partial\rangle / \mathbb{C}[\hbar] \llbracket q \rrbracket I_{\text {poly }}\right)$.

(vi) The generalized mirror transformation can be done using only convergent power series i.e. $\Omega_{a}(q, \hbar)$ and $g(q, \hbar)$ in the step (i) and (ii) of Theorem 5.6 are convergent functions of $q$ and $\hbar$. 
Proof. (i) $\Leftrightarrow$ (ii): The condition $c_{1}(X) \geq 0$ is equivalent to that every primitive generator $\vec{x}_{i}$ of one-dimensional cones lies in the boundary of $\operatorname{Conv}\left(\vec{x}_{1}, \ldots, \vec{x}_{r+N}\right)$. The number of top dimensional cones in $\Sigma$ equals $\operatorname{dim}_{\mathbb{C}} H^{*}(X)$ and each top dimensional cone has volume $1 / N$ !. Hence we obtain the equivalence.

(ii) $\Leftrightarrow$ (iii): This follows from Proposition 5.11

(iii) $\Rightarrow$ (iv): Set $M:=\mathcal{O}_{\text {small }}^{\hbar}\langle\hbar \partial\rangle / \mathcal{O}_{\text {small }}^{\hbar} I_{\text {poly }}$. Let $[f(q, \hbar \partial, \hbar)]$ be an element of the kernel of the natural surjection $M \rightarrow \widetilde{F H}_{S^{1}}^{*}$. We can assume that $f(q, \hbar \partial, \hbar)$ is homogeneous. By the assumption, we can see that there exists an integer $k>0$ such that $\left(q_{1} \cdots q_{r}\right)^{k} f(q, \mathrm{p}, 0)$ is contained in $\sigma\left(\mathcal{O}_{\text {small }}^{\hbar} I_{\text {poly }}\right)$. Because $\sigma\left(\mathcal{O}_{\text {small }}^{\hbar} I_{\text {poly }}\right)=\sum_{\boldsymbol{d}} \mathbb{C}\{q\}\left[q^{-1}\right] \sigma\left(\mathcal{P}_{\boldsymbol{d}}\right) \cap \mathbb{C}\{q\}$, we can see that $f(q, \mathrm{p}, 0)$ is also in $\sigma\left(\mathcal{O}_{\text {poly }}^{\hbar} I_{\text {poly }}\right)$. Therefore, there exists some $f_{1}(q, \hbar \partial, \hbar)$ in $\mathcal{O}_{\text {small }}^{\hbar}\langle\hbar \partial\rangle$ such that $f(q, \hbar \partial, \hbar) \equiv \hbar f_{1}(q, \hbar \partial, \hbar) \bmod \mathcal{O}_{\text {small }}^{\hbar} I_{\text {poly }}$ and $\operatorname{deg} f_{1}=\operatorname{deg} f-2$. Then, [ $\left[\hbar f_{1}\right]$ is in the kernel of $M \rightarrow \widetilde{F H}_{S^{1}}^{*}$. Because the multiplication by $\hbar$ is injective in $\widetilde{F H}_{S^{1}}^{*},\left[f_{1}\right]$ also lies in the kernel. Repeating this, we have $f \equiv \hbar^{n} f_{n} \bmod \mathcal{O}_{\text {small }}^{\hbar} I_{\text {poly }}$ and $\operatorname{deg} f_{n}<0$ for sufficiently large $n$. Because we have already seen that (i) is equivalent to (iii), $c_{1}(X)$ is nef and so $\operatorname{deg} q_{a} \geq 0$. Thus $f_{n}$ must be zero and $[f]=0$ in $M$.

(iv) $\Rightarrow(\mathrm{v})$ : Take the tensor product with $\mathbb{C}[\hbar] \llbracket q \rrbracket$.

(v) $\Rightarrow$ (ii): Because $F H_{S^{1}}^{*}$ is a free $\mathbb{C}[\hbar] \llbracket q \rrbracket$-module of $\operatorname{rank} \operatorname{dim}_{\mathbb{C}} H^{*}(X)$, we see that $\sigma\left(F H_{0}\right) \otimes_{\mathbb{C}[q]} \mathbb{C} \llbracket q \rrbracket$ is a free $\mathbb{C} \llbracket q \rrbracket$-module of $\operatorname{rank} \operatorname{dim}_{\mathbb{C}} H^{*}(X)$. On the other hand, by Proposition $5.11 \sigma\left(F H_{0}\right)\left[q^{-1}\right] \cong \sigma\left(M_{\text {Mell }}\right)$ becomes a free module of rank $N$ ! $\operatorname{Vol}(\operatorname{Conv}(\Sigma))$ when restricted to the complement of the ramification locus. Therefore, we must have $\operatorname{dim}_{\mathbb{C}} H^{*}(X)=$ $N ! \operatorname{Vol}(\operatorname{Conv}(\Sigma))$.

(i) $\Leftrightarrow(\mathrm{v})$ : We can see from the proof of Proposition [5.5 that the $I$-function in (28) is convergent on $|\hbar|=1$ if and only if $c_{1}(X)$ is nef. The connection matrix $\Omega_{a}$ is determined from $I$-function by (27). The gauge transformation $g$ can be found by applying Birkhoff factorization of the loop group $L G L(s+1, \mathbb{C})$ to the loop $\hbar \mapsto\left(T_{i}(\hbar \partial) I(q, \hbar)\right)$ (see [14, 16, 17]).

5.5. Example. We study the case of Hirzeburch surface $\mathbb{F}_{n}=\mathbb{P}\left(\mathcal{O}_{\mathbb{P}^{1}} \oplus \mathcal{O}_{\mathbb{P}^{1}}(n)\right)$. This is given by the fan $\Sigma$ whose one-dimensional cones are generated by $\vec{x}_{1}, \ldots, \vec{x}_{4}$ :

$$
\vec{x}_{1}=(1,0), \quad \vec{x}_{2}=(0,1), \quad \vec{x}_{3}=(-1, n), \quad \vec{x}_{4}=(0,-1) .
$$

We have $\operatorname{dim}_{\mathbb{C}} H^{*}\left(\mathbb{F}_{n}\right)=4$ and $2 ! \operatorname{Vol}(\operatorname{Conv}(\Sigma))=\max (4, n+2)$. Let $w_{1}, \ldots, w_{4}$ be the classes of corresponding toric divisors. Then, $p_{1}:=w_{1}$ and $p_{2}:=w_{4}$ form a nef integral basis and we have $w_{2}=p_{2}-n p_{1}$ and $w_{3}=p_{1}$. The relation of classical cohomology ring is given by $p_{1}^{2}=0$ and $p_{2}^{2}=n p_{1} p_{2}$. The corresponding Mellin system is generated by the following two differential operators:

$$
\mathcal{P}_{(1,0)}=q_{1} W_{2}\left(W_{2}-\hbar\right) \cdots\left(W_{2}-(n-1) \hbar\right)-W_{1} W_{3}, \quad \mathcal{P}_{(0,1)}=q_{2}-W_{4} W_{2},
$$

where $W_{1}=W_{3}=\hbar \partial_{1}, W_{2}=\hbar \partial_{2}-n \hbar \partial_{1}, W_{4}=\hbar \partial_{2}$.

$$
\begin{gathered}
F H_{0} \cong \mathbb{C}\left\langle q_{1}, q_{2}, \hbar \partial_{1}, \hbar \partial_{2}, \hbar\right\rangle /\left\langle\mathcal{P}_{(1,0)}, \mathcal{P}_{(0,1)}\right\rangle, \quad M_{\text {Mell }} \cong F H_{0}\left[q_{1}^{-1}, q_{2}^{-2}\right] \\
F H_{S^{1}}^{*} \cong \mathbb{C}[\hbar] \llbracket q_{1}, q_{2} \rrbracket\left\langle\hbar \partial_{1}, \hbar \partial_{2}\right\rangle / \overline{\left\langle\mathcal{P}_{(1,0)}, \mathcal{P}_{(0,1)}\right\rangle}
\end{gathered}
$$

5.5.1. Fano case $(n=0,1)$. In this case, $F H_{0}$ is freely generated by $[1],\left[\hbar \partial_{1}\right],\left[\hbar \partial_{2}\right],\left[\left(\hbar \partial_{2}\right)^{2}\right]$ over $\mathbb{C}[\hbar, q]$. The small quantum $D$-module of $\mathbb{F}_{n}$ is defined over the polynomial ring $\mathbb{C}[\hbar, q]$ and is isomorphic to $\mathrm{FH}_{0}$. 
5.5.2. Nef but non-Fano case $(n=2)$. In this case, $F H_{0}$ is not finitely generated over $\mathbb{C}[\hbar, q]$. However, $F H_{0}\left[\left(1-4 q_{1}\right)^{-1}\right]$ is freely generated by $[1],\left[\hbar \partial_{1}\right],\left[\hbar \partial_{2}\right],\left[\left(\hbar \partial_{2}\right)^{2}\right]$ over $\mathbb{C}\left[\hbar, q_{1}, q_{2},(1-\right.$ $\left.\left.4 q_{1}\right)^{-1}\right]$. For example, we can write $\left[\left(\hbar \partial_{1}\right)^{2}\right]$ as

$$
\left[\left(\hbar \partial_{1}\right)^{2}\right]=\frac{2 q_{1} q_{2}}{1-4 q_{1}}+\frac{2 \hbar q_{1}}{1-4 q_{1}}\left[\hbar \partial_{1}\right]-\frac{\hbar q_{1}}{1-4 q_{1}}\left[\hbar \partial_{2}\right]-\frac{q_{1}}{1-4 q_{1}}\left[\left(\hbar \partial_{2}\right)^{2}\right]
$$

The small quantum $D$-module is isomorphic to $F H_{0}$ at least on the region $\left|4 q_{1}\right|<1$ after a suitable coordinate change (see [14]).

5.5.3. Non-nef case $(n \geq 3)$. In this case, $F H_{0}$ is not finitely generated over $\mathbb{C}[\hbar, q]$. Furthermore, $F H_{0} \otimes_{\mathbb{C}[\hbar, q]} \mathbb{C}[\hbar] \llbracket q \rrbracket \cong \mathbb{C}[\hbar] \llbracket q \rrbracket\langle\hbar \partial\rangle /\left\langle\mathcal{P}_{(1,0)}, \mathcal{P}_{(0,1)}\right\rangle$ is not finitely generated over $\mathbb{C}[\hbar] \llbracket q \rrbracket$ either. If it were finitely generated, then $\sigma\left(F H_{0}\right) \otimes_{\mathbb{C}[q]} \mathbb{C} \llbracket q \rrbracket$ would be also finitely generated over $\mathbb{C} \llbracket q \rrbracket$ and generated by $1, \mathrm{p}_{1}, \mathrm{p}_{2}, \mathrm{p}_{2}^{2}$ by Nakayama's lemma. It contradicts that $\sigma\left(F H_{0}\right)\left[q_{1}^{-1}, q_{2}^{-1}\right] \cong \sigma\left(M_{\text {Mell }}\right)$ is a free module of rank $n+2>4$. We must consider the $q$-adic closure of the left ideal $\left\langle\mathcal{P}_{(1,0)}, \mathcal{P}_{(0,1)}\right\rangle$ which is strictly bigger:

$$
\left\langle\mathcal{P}_{(1,0)}, \mathcal{P}_{(0,1)}\right\rangle \varsubsetneqq \overline{\left\langle\mathcal{P}_{(1,0)}, \mathcal{P}_{(0,1)}\right\rangle} \quad \text { in } \mathbb{C}[\hbar] \llbracket q \rrbracket\langle\hbar \partial\rangle .
$$

Our $F H_{S^{1}}^{*}$ is a free $\mathbb{C}[\hbar] \llbracket q \rrbracket$-module of rank 4 and isomorphic to the restriction of the big quantum $D$-module to some non-linear subvariety of $H^{*}\left(\mathbb{F}_{n}\right)$. On the other hand, $M_{\mathrm{Mell}}$ is of rank $n+2$ over $\mathbb{C}\left[\hbar, q^{ \pm}\right]$. (In fact, it is generated by $[1],\left[\hbar \partial_{1}\right],\left[\left(\hbar \partial_{1}\right)^{2}\right],\left[\hbar \partial_{2}-n \hbar \partial_{1}\right], \ldots,\left[\left(\hbar \partial_{2}-\right.\right.$ $\left.\left.n \hbar \partial_{1}\right)^{n-1}\right]$.) The characteristic variety of $M_{\text {Mell }}$ is given by

$$
q_{1}\left(\mathrm{p}_{2}-n \mathrm{p}_{1}\right)^{n}=\mathrm{p}_{1}^{2}, \quad q_{2}=\left(\mathrm{p}_{2}-n \mathrm{p}_{1}\right) \mathrm{p}_{2}
$$

or equivalently,

$$
n^{2} q_{1} q_{2}^{n}=\mathrm{p}_{2}^{n-2}\left(\mathrm{p}_{2}^{2}-q_{2}\right)^{2}, \quad q_{2}=\left(\mathrm{p}_{2}-n \mathrm{p}_{1}\right) \mathrm{p}_{2} .
$$

It has $n+2$ solutions $\left(\mathrm{p}_{1}, \mathrm{p}_{2}\right)$ (counted with multiplicity) for a given $\left(q_{1}, q_{2}\right) \in\left(\mathbb{C}^{*}\right)^{2}$. Out of $n+2$ solutions, we have four branches of solutions having the asymptotics:

$$
\mathrm{p}_{1} \sim\left(q_{1}\left(\sqrt{q_{2}}\right)^{n}\right)^{1 / 2}, \quad \mathrm{p}_{2} \sim \sqrt{q_{2}}+\frac{n}{2}\left(q_{1}\left(\sqrt{q_{2}}\right)^{n}\right)^{1 / 2} .
$$

These branches satisfy $\left(\mathrm{p}_{1}, \mathrm{p}_{2}\right) \rightarrow 0$ as $\left(q_{1}, q_{2}\right) \rightarrow 0$ and correspond to the characteristic variety of $\widetilde{F H}_{S^{1}}^{*}$. The other $n-2$ branches have the asymptotics

$$
\mathrm{p}_{1} \sim-n^{\frac{n-4}{n-2}} q_{1}^{-\frac{1}{n-2}}, \quad \mathrm{p}_{2} \sim n^{\frac{2}{n-2}} q_{1}^{\frac{1}{n-2}} q_{2}
$$

and diverge as $q_{1} \rightarrow 0$.

By changing the large radius limit $q \rightarrow 0$, we can construct a $D$-module of rank $n+2$ which is regular singular along $\hat{q}_{1} \hat{q}_{2}=0$. Set $\hat{q}_{1}=q_{1}^{-1}$ and $\hat{q}_{2}=q_{2} q_{1}$. In terms of $\hat{q}_{1}, \hat{q}_{2}$, differential operators of the Mellin system are written as

$$
\begin{gathered}
\mathcal{R}_{(1,0)}=\hat{q}_{1} W_{1} W_{3}-W_{2}\left(W_{2}-\hbar\right) \cdots\left(W_{2}-(n-1) \hbar\right), \quad \mathcal{R}_{(1,1)}=\hat{q}_{1} \hat{q}_{2}-W_{2} W_{4}, \\
\mathcal{R}_{(0,1)}=\hat{q}_{2} W_{2}\left(W_{2}-\hbar\right) \cdots\left(W_{2}-(n-2) \hbar\right)-W_{1} W_{3} W_{4} .
\end{gathered}
$$

Then, the $D$-module

$$
F H_{0}^{\prime}=\mathbb{C}\left\langle\hat{q}_{1}, \hat{q}_{2}, \hbar \partial_{1}, \hbar \partial_{2}, \hbar\right\rangle /\left\langle\mathcal{R}_{(1,0)}, \mathcal{R}_{(1,1)}, \mathcal{R}_{(0,1)}\right\rangle
$$

is freely generated by $[1],\left[\hbar \partial_{1}\right],\left[\left(\hbar \partial_{1}\right)^{2}\right],\left[\hbar \partial_{2}-n \hbar \partial_{1}\right], \cdots,\left[\left(\hbar \partial_{2}-n \hbar \partial_{1}\right)^{n-1}\right]$ as a $\mathbb{C}\left[\hbar, \hat{q}_{1}, \hat{q}_{2}\right]$ module. This becomes an abstract quantum $D$-module in the sense of [16, 17]. Therefore, we can find a canonical frame and flat coordinates by reconstructing an $n+2$-dimensional base space (see [17]). It would be interesting to study a geometric meaning of this $D$-module. 


\section{6. $R$-CONJECTURE (VIRASORO CONSTRAints)}

The Virasoro constraints are infinite dimensional symmetries of the (all genus, descendant) Gromov-Witten potential conjectured by Eguchi-Hori-Xiong [6]. In [13, Givental showed that when the target $X$ has a torus action with only isolated fixed points and isolated onedimensional orbits, the Virasoro conjecture is reduced to the $R$-conjecture. The $R$-conjecture is a conjecture about equivariant quantum cohomology with semisimple non-equivariant counterpart. It states that the asymptotic solution $R$ defined in the equivariant theory has nonequivariant limit. For a class of Fano toric varieties, the $R$-conjecture was proved by Givental [13. The $R$-conjecture has been proved for complete flag [20], Grassmannian [2] and partial flag varieties 3 .

In this section, we explain the $R$-conjecture and prove it for any smooth projective toric variety. The proof of $R$-conjecture will be a first step for the understanding of the mirror oscillatory integral.

6.1. Equivariant quantum cohomology. Let $\mathbb{T}$ be an $l$-dimensional torus $\left(\cong\left(S^{1}\right)^{l}\right)$ and $X$ be a $\mathbb{T}$-manifold. In a manner analogous to section 2, we can define the equivariant quantum cohomology algebra $\left(Q H_{\mathbb{T}}^{*}(X), *\right)$ which is a deformation of the ring structure of the ordinary equivariant cohomology $H_{\mathbb{T}}^{*}(X)[9$. We assume that the $\mathbb{T}$-action on $X$ is Hamiltonian. For projective manifolds, this assumption is equivalent to that the action has at least one fixed point. Then, the equivariant cohomology is of the form $H_{\mathbb{T}}^{*}(X) \cong H^{*}(X) \otimes H_{\mathbb{T}}^{*}(\mathrm{pt})$. This isomorphism is not canonical, but we choose homogeneous equivariant lifts $p_{0}, \ldots, p_{s}$ of the basis in section 2. Let $t_{0}, \ldots, t_{s}$ be linear coordinates dual to $p_{0}, \ldots, p_{s}$ and put $H_{\mathbb{T}}^{*}(\mathrm{pt})=\mathbb{C}\left[\lambda_{1}, \ldots, \lambda_{l}\right]$. Set $q_{a}=\exp \left(t_{a}\right)$ for $1 \leq a \leq r$. The equivariant quantum cohomology is of the form

$$
Q H_{\mathbb{T}}^{*}(X)=H_{\mathbb{T}}^{*}(X) \widehat{\otimes} \mathbb{C} \llbracket t_{0}, q_{1}, \ldots, q_{r}, t_{r+1}, \ldots, t_{s} \rrbracket=H^{*}(X) \otimes \mathbb{C}[\lambda] \llbracket x \rrbracket .
$$

Here, $\mathbb{C}[\lambda] \llbracket x \rrbracket$ is shorthand for $\mathbb{C}\left[\lambda_{1}, \ldots, \lambda_{l}\right] \llbracket t_{0}, q_{1}, \ldots, q_{r}, t_{r+1}, \ldots, t_{s} \rrbracket$ and $\widehat{\otimes}$ means the tensor product completed in the $x$-adic topology.

Our $\left(Q H_{\mathbb{T}}^{*}(X), *\right)$ defines a quasi-conformal Frobenius structure [1] on the base space $\mathcal{M}=$ $\operatorname{Spec}\left(\mathbb{C}(\lambda) \llbracket x \rrbracket\left[q_{1}^{-1}, \ldots, q_{r}^{-1}\right]\right)$. Let $\mathcal{T}_{\mathcal{M}}$ be the relative tangent sheaf of $\mathcal{M} \rightarrow \operatorname{Spec} \mathbb{C}(\lambda)$. Then, each element of $Q H_{\mathbb{T}}^{*}(X)$ gives a section of $\mathcal{T}_{\mathcal{M}}$ by the correspondence $p_{i} \mapsto \partial_{i}:=\partial / \partial t_{i}$. The quasi-conformal Frobenius structure of $Q H_{\mathbb{T}}^{*}(X)$ consists of the following data:

(1) Flat $\mathbb{C}(\lambda)$-bilinear symmetric pairing $\langle\cdot, \cdot\rangle_{\mathbb{T}}$ on $\mathcal{T}_{\mathcal{M}}$ defined by $\left\langle\partial_{i}, \partial_{j}\right\rangle_{\mathbb{T}}=\int_{X}^{\mathbb{T}} p_{i} \cup p_{j}$.

(2) $\mathbb{C}(\lambda)$-bilinear symmetric product $*: \mathcal{T}_{\mathcal{M}} \otimes \mathcal{T}_{\mathcal{M}} \rightarrow \mathcal{T}_{\mathcal{M}}$ satisfying $\left\langle\partial_{i} * \partial_{j}, \partial_{k}\right\rangle_{\mathbb{T}}=\left\langle\partial_{i}, \partial_{j} * \partial_{k}\right\rangle_{\mathbb{T}}$.

(3) Flat unit section $1=p_{0}=\partial_{0} \in \mathcal{T}_{\mathcal{M}}$.

(4) Euler operator $\widetilde{E}$ defined by

$$
\widetilde{E}=2 t_{0} \frac{\partial}{\partial t_{0}}+2 c_{0}(\lambda) \frac{\partial}{\partial t_{0}}+\sum_{a=1}^{r} 2 c_{a} \frac{\partial}{\partial t_{a}}+\sum_{j=r+1}^{s}\left(2-\operatorname{deg} p_{j}\right) t_{j} \frac{\partial}{\partial t_{j}}+\sum_{j=1}^{l} 2 \lambda_{j} \frac{\partial}{\partial \lambda_{j}}
$$

where $c_{0}(\lambda)+\sum_{a=1}^{r} c_{a} p_{a}=c_{1}^{\mathbb{T}}(T X)$.

The name quasi-conformal comes from that $\widetilde{E}$ is not a section of $\mathcal{T}_{\mathcal{M}}$ (it contains the derivation $\left.\lambda_{j} \partial / \partial \lambda_{j}\right)$. As in non-equivariant case, the dual Givental connection $\nabla_{j}^{\hbar}=\hbar\left(\partial / \partial t_{j}\right)+p_{j} *$ on $\mathcal{T}_{\mathcal{M}}$ is flat for any value of $\hbar$. This defines the $\mathbb{T}$-equivariant quantum $D$-module:

$$
Q D M_{\mathbb{T}}^{*}(X)=\left(H^{*}(X) \otimes \mathbb{C}[\lambda, \hbar] \llbracket x \rrbracket, \nabla^{\hbar}\right) .
$$


The Euler operator satisfies the following:

$$
\begin{aligned}
& {\left[\widetilde{E}, V_{1} * V_{2}\right]=V_{1} *\left[\widetilde{E}, V_{2}\right]+\left[\widetilde{E}, V_{1}\right] * V_{2}+2 V_{1} * V_{2},} \\
& \widetilde{E}\left\langle V_{1}, V_{2}\right\rangle_{\mathbb{T}}=\left\langle\left[\widetilde{E}, V_{1}\right], V_{2}\right\rangle_{\mathbb{T}}+\left\langle V_{1},\left[\widetilde{E}, V_{2}\right]\right\rangle_{\mathbb{T}}+(4-2 N)\left\langle V_{1}, V_{2}\right\rangle_{\mathbb{T}},
\end{aligned}
$$

for $V_{1}, V_{2} \in \mathcal{T}_{\mathcal{M}}$ and $N=\operatorname{dim}_{\mathbb{C}} X$.

We assume some familiarity with the semisimplicity and canonical coordinates for conformal Frobenius manifolds, see e.g. 5, 24]. Here, we review the construction of canonical coordinates in equivariant quantum cohomology. Assume that the $\mathbb{T}$ action on $X$ has only isolated fixed points. Then, by the localization theorem of equivariant cohomology, we have an isomorphism of rings

$$
H_{\mathbb{T}}^{*}(X) \otimes_{\mathbb{C}[\lambda]} \mathbb{C}(\lambda) \cong \bigoplus_{\sigma \in X^{\mathbb{T}}} \mathbb{C}(\lambda) \psi_{\sigma}, \quad \psi_{\sigma}=\frac{i_{\sigma *}([\sigma])}{\boldsymbol{e}^{\mathbb{T}}\left(T_{\sigma} X\right)} .
$$

Here, $i_{\sigma}:\{\sigma\} \rightarrow X$ is the inclusion and $\boldsymbol{e}^{\mathbb{T}}$ is the $\mathbb{T}$-equivariant Euler class. Because $i_{\sigma}^{*}\left(\psi_{\tau}\right)=$ $\delta_{\sigma \tau}$ for $\sigma, \tau \in X^{\mathbb{T}}$, we have $\psi_{\sigma} \cup \psi_{\tau}=\delta_{\sigma \tau} \psi_{\sigma}$. The localized equivariant quantum cohomology is also semisimple. The idempotent $\psi_{\sigma}$ can be deformed to the idempotent $\psi_{\sigma}(x)$ of $Q H_{\mathbb{T}}^{*}(X) \widehat{\otimes}_{\mathbb{C}[\lambda]} \mathbb{C}(\lambda)$ such that $\lim _{q \rightarrow 0} \psi_{\sigma}(x)=\psi_{\sigma}$ and $\psi_{\sigma}(x) * \psi_{\tau}(x)=\delta_{\sigma \tau} \psi_{\sigma}(x)$. The projection to the $\psi_{\sigma}(x)$-component $Q H_{\mathbb{T}}^{*}(X) \widehat{\otimes}_{\mathbb{C}[\lambda]} \mathbb{C}(\lambda) \rightarrow \mathbb{C}(\lambda) \llbracket x \rrbracket$ corresponds to a closed 1-form on $\mathcal{M}$. A primitive $\mathrm{u}_{\sigma}(t, \lambda)$ of this closed 1 -form is called a canonical coordinate. Then, we can write $\psi_{\sigma}(x)=\partial / \partial \mathbf{u}_{\sigma}$. Note that $d \mathbf{u}_{\sigma}$ is characterized by

$$
V * \psi_{\sigma}(x)=d \mathbf{u}_{\sigma}(V) \psi_{\sigma}(x), \quad V \in \mathcal{T}_{\mathcal{M}} .
$$

The canonical coordinates $\left\{\mathbf{u}_{\sigma}\right\}_{\sigma \in X^{\mathbb{T}}}$ are determined up to functions in $\lambda_{i}$.

Put $\alpha(\sigma)=i_{\sigma}^{*}(\alpha)$ for $\alpha \in H_{\mathbb{T}}^{*}(X)$. Let $T_{\sigma}^{*} X \cong \chi_{1}(\sigma) \oplus \cdots \oplus \chi_{N}(\sigma)$ be the weight decomposition, where $\chi_{j}(\sigma) \in \mathbb{C}[\lambda]$. Although $\mathcal{M}$ does not contain the divisor $q_{a}=0$, the $\operatorname{limit} \lim _{q \rightarrow 0} d \mathbf{u}_{\sigma}$ exists as an element of $\left(H^{*}(X)\right)^{*} \otimes \mathbb{C}(\lambda)$ because the dual basis $\psi_{\sigma}(x)$ is in $H^{*}(X) \otimes \mathbb{C}(\lambda) \llbracket x \rrbracket$. Since $\lim _{q \rightarrow 0} d \mathbf{u}_{\sigma}$ is the projection to $\psi_{\sigma}$, we have $d \mathbf{u}_{\sigma}=\sum_{i=0}^{s}\left(p_{i}(\sigma)+O(q)\right) d t_{i}$, where $O(q) \in$ $\mathbb{C}(\lambda) \llbracket x \rrbracket$. We normalize canonical coordinates by the following classical limit condition:

$$
\mathrm{u}_{\sigma}(t, \lambda)=\sum_{j=1}^{N}\left(-\chi_{j}(\sigma)+\chi_{j}(\sigma) \log \left(-\chi_{j}(\sigma)\right)\right)+\sum_{i=0}^{s} p_{i}(\sigma) t_{i}+O(q)
$$

where $O(q) \in \mathbb{C}(\lambda) \llbracket x \rrbracket$. This together with (33) determines $\mathbf{u}_{\sigma}(t, \lambda)$ uniquely. Using $c_{1}^{\mathbb{T}}\left(T_{\sigma} X\right)=$ $c_{0}(\lambda)+\sum_{a=1}^{r} c_{a} p_{a}(\sigma)=-\sum_{j=1}^{N} \chi_{j}(\sigma)$, we can check that this $\mathbf{u}_{\sigma}$ automatically satisfies the homogeneity $\widetilde{E} \mathbf{u}_{\sigma}=2 \mathrm{u}_{\sigma}$.

Assume that $Q H_{\mathbb{T}}^{*}(X)$ has convergent structure constants around $x=\lambda=0$. Then $\mathrm{u}_{\sigma}(t, \lambda)$ becomes a multi-valued analytic function. We conjecture the following which resembles the $R$-conjecture.

Conjecture 6.1. When the non-equivariant quantum cohomology is generically semisimple, canonical coordinates $\mathrm{u}_{\sigma}(t, \lambda)$ normalized by 34) is regular at $\lambda=0$ for a semisimple point $t$ of non-equivariant theory.

Let $\mathbf{u}_{\sigma}^{\prime}(t, \lambda)$ be any canonical coordinate which is homogeneous $\widetilde{E} \mathbf{u}_{\sigma}^{\prime}=2 \mathbf{u}_{\sigma}^{\prime}$ and is regular at $\lambda=0$ for semisimple $t$. If this conjecture is true, such a canonical coordinate is different from the above normalization only by a linear form $\sum_{i=1}^{l} a_{i} \lambda_{i}$. Note that the non-equivariant limit $\lambda \rightarrow 0$ of $\mathrm{u}_{\sigma}(t, \lambda)$ gives the canonical coordinate $\mathrm{u}(t)$ of non-equivariant theory satisfying the homogeneity $E \mathrm{u}(t)=2 \mathrm{u}(t)$. (In non-equivariant theory, homogeneous canonical coordinates 
are unique up to order.) Later, we will see that this conjecture (together with $R$-conjecture stated below) holds for toric variety using the equivariant mirror.

Consider the following differential equation for a section $s(t, \lambda, \hbar) \in \mathcal{T}_{\mathcal{M}}$.

$$
\hbar \frac{\partial}{\partial t_{i}} s(t, \lambda, \hbar)=p_{i} * s(t, \lambda, \hbar) .
$$

An asymptotic solution [5, 11, 12, 13, $s_{\tau}(t, \lambda, \hbar)$ for this differential equation is the solution of the form

$$
s_{\tau}(t, \lambda, \hbar)=\sum_{\sigma} R_{\sigma \tau}(t, \lambda, \hbar) e^{\mathbf{u}_{\tau}(t, \lambda) / \hbar} \triangle_{\sigma} \frac{\partial}{\partial \mathbf{u}_{\sigma}}, \quad \triangle_{\sigma}=\left\langle\frac{\partial}{\partial \mathbf{u}_{\sigma}}, \frac{\partial}{\partial \mathbf{u}_{\sigma}}\right\rangle_{\mathbb{T}}^{-1 / 2},
$$

where $R_{\sigma \tau}(t, \lambda, \hbar)=\delta_{\sigma \tau}+R_{\sigma \tau}^{(1)}(t, \lambda) \hbar+R_{\sigma \tau}^{(2)}(t, \lambda) \hbar^{2}+\cdots$ is an asymptotic series in $\hbar$. The matrix $R_{\sigma \tau}$ is uniquely determined by the classical limit condition:

$$
\lim _{q \rightarrow 0} R_{\sigma \tau}(t, \lambda, \hbar)=\delta_{\sigma \tau} \exp \left(b_{\sigma}(\hbar, \lambda)\right), \quad b_{\sigma}(\hbar, \lambda)=\sum_{k=1}^{\infty} N_{2 k-1}(\sigma) \frac{B_{2 k}}{2 k} \frac{\hbar^{2 k-1}}{2 k-1},
$$

where $N_{2 k-1}(\sigma)=\sum_{j=1}^{N} 1 / \chi_{j}(\sigma)^{2 k-1}$. Since $\lim _{q \rightarrow 0} \triangle_{\sigma}=\boldsymbol{e}^{\mathbb{T}}\left(T_{\sigma} X\right)^{1 / 2}$, we can see that $R_{\sigma \tau}^{(n)}(t, \lambda)$ belongs to $\mathbb{C}\left(\lambda, e^{\mathbb{T}}\left(T_{\sigma} X\right)^{1 / 2}\right) \llbracket x \rrbracket$. Under this normalization, $R_{\sigma \tau}$ automatically satisfies the following homogeneity and the unitarity:

$$
\left(2 \hbar \frac{\partial}{\partial \hbar}+\widetilde{E}\right) R_{\sigma \tau}(t, \lambda, \hbar)=0, \quad \sum_{\nu \in X^{\mathbb{T}}} R_{\nu \sigma}(t, \lambda,-\hbar) R_{\nu \tau}(t, \lambda, \hbar)=\delta_{\sigma \tau} .
$$

Conjecture 6.2 (R-conjecture [13]). When the non-equivariant quantum cohomology is generically semisimple, the asymptotic solution $R_{\sigma \tau}^{(n)}(t, \lambda)$ normalized by (36) is regular at $\lambda=0$ for a semisimple point $t$ of non-equivariant theory.

An asymptotic solution $R_{\sigma \tau}(t, \lambda, \hbar)$ satisfying the homogeneity and regularity at $\lambda=0$ for a semisimple $t$ is unique if it exists. Thus, if $R$-conjecture is true, such a solution exists and satisfies the above classical limit condition (36). In this case, the non-equivariant limit $\lim _{\lambda \rightarrow 0} R_{\sigma \tau}$ gives the homogeneous asymptotic solution of non-equivariant theory.

By the theory of Givental [12, 13, the $R$-conjecture implies the Virasoro constraints for the non-equivariant Gromov-Witten theory of $X$.

6.2. Equivariant mirror. We review an equivariant version of the mirror of toric variety 10. We use the same notation as in section [5.1. The torus $\mathbb{T}:=\left(S^{1}\right)^{r+N}$ acts on a toric variety $X=\mathbb{C}_{\mathcal{B}}^{r+N} /\left(\mathbb{C}^{*}\right)^{r}$ by $\left(z_{1}, \ldots, z_{r+N}\right) \mapsto\left(t_{1} z_{1}, \ldots, t_{r+N} z_{r+N}\right)$. Here, $w_{1}, \ldots, w_{r} \in H_{\mathbb{T}}^{2}(X)$ denotes the $\mathbb{T}$-equivariant Poincaré duals of torus invariant prime divisors $D_{1}, \ldots, D_{r+N}$. In other words, $w_{i}$ is the $\mathbb{T}$-equivariant first Chern class of the line bundle

$$
\mathbb{C}_{\mathcal{B}}^{r+N} \times \mathbb{C} /\left(z_{1}, \ldots, z_{r+N}, v\right) \sim\left(t^{w_{1}} z_{1}, \ldots, t^{w_{r+N}} z_{r+N}, t^{w_{i}} v\right),\left(t_{1}, \ldots, t_{r}\right) \in\left(\mathbb{C}^{*}\right)^{r}
$$

with $\mathbb{T}$-action $\left(z_{1}, \ldots, z_{r+N}, v\right) \mapsto\left(t_{1} z_{1}, \ldots, t_{r+N} z_{r+N}, t_{i} v\right)$. Let $p_{a}$ denote an equivariant lift of the nef integral basis in section [5.1. More precisely, we define $p_{a}$ as the $\mathbb{T}$-equivariant first Chern class of the line bundle

$$
\mathbb{C}_{\mathcal{B}}^{r+N} \times \mathbb{C} /\left(z_{1}, \ldots, z_{r+N}, v\right) \sim\left(t^{w_{1}} z_{1}, \ldots, t^{w_{r+N}} z_{r+N}, t_{a} v\right),\left(t_{1}, \ldots, t_{r}\right) \in\left(\mathbb{C}^{*}\right)^{r} .
$$

with $\mathbb{T}$-action $\left(z_{1}, \ldots, z_{r+N}, v\right) \mapsto\left(t_{1} z_{1}, \ldots, t_{r+N} z_{r+N}, v\right)$. Then, $w_{i}$ is written as

$$
w_{i}=\sum_{a=1}^{r} m_{i a} p_{a}-\lambda_{j}
$$


Define an equivariant phase function $F^{\mathbb{T}}(\mathrm{w} ; \lambda)$ on the mirror family $\pi: Y \rightarrow\left(\mathbb{C}^{*}\right)^{r}$ by $F^{\mathbb{T}}(\mathrm{w} ; \lambda)=$ $\sum_{i=1}^{r+N}\left(\mathrm{w}_{i}+\lambda_{i} \log \mathrm{w}_{i}\right)$. Set $F_{q}^{\mathbb{T}}=\left.F^{\mathbb{T}}\right|_{Y_{q}}$. Then consider the equivariant oscillatory integral:

$$
\mathcal{I}_{\Gamma}(q, \lambda, \hbar)=\int_{\Gamma_{q} \subset Y_{q}} e^{F_{q}^{\mathbb{T}} / \hbar} \omega_{q}
$$

We can easily obtain the equivariant version of Proposition 5.1

Proposition 6.3. The equivariant oscillatory integral $\mathcal{I}_{\Gamma}$ satisfies $\mathcal{P}_{\boldsymbol{d}}^{\mathbb{T}} \mathcal{I}_{\Gamma}(q, \lambda, \hbar)=0$ for all $\boldsymbol{d} \in \mathbb{Z}^{r}$, where

$$
\mathcal{P}_{\boldsymbol{d}}^{\mathbb{T}}=q^{\boldsymbol{d}} \prod_{\left\langle w_{i}, \boldsymbol{d}\right\rangle<0} \prod_{k=0}^{-\left\langle w_{i}, \boldsymbol{d}\right\rangle-1}\left(\sum_{a=1}^{r} m_{i a} \hbar \partial_{a}-\lambda_{i}-k \hbar\right)-\prod_{\left\langle w_{i}, \boldsymbol{d}\right\rangle>0} \prod_{k=0}^{\left\langle w_{i}, \boldsymbol{d}\right\rangle-1}\left(\sum_{a=1}^{r} m_{i a} \hbar \partial_{a}-\lambda_{i}-k \hbar\right) .
$$

We introduce the $D$-module $M_{\text {Mell }}^{\mathbb{T}}$ as in section 5.1 .

$$
M_{\mathrm{Mell}}^{\mathbb{T}}=\mathbb{C}\left\langle q^{ \pm}, \hbar \partial, \hbar, \lambda\right\rangle / I_{\text {Mell }}^{\mathbb{T}}, \quad I_{\text {Mell }}^{\mathbb{T}}=\sum_{\boldsymbol{d} \in \mathbb{Z}^{r}} \mathbb{C}\left\langle q^{ \pm}, \hbar \partial, \hbar, \lambda\right\rangle \mathcal{P}_{\boldsymbol{d}}^{\mathbb{T}}
$$

6.3. $\mathbb{T} \times S^{1}$-equivariant Floer cohomology. We can construct $\mathbb{T} \times S^{1}$-equivariant Floer cohomology $F H_{\mathbb{T} \times S^{1}}^{*}$ in an analogous manner to section [5.2. First, $H_{\mathbb{T} \times S^{1}}^{\infty / 2}$ is defined by the limit of the inductive system $\left(H_{\mathbb{T} \times S^{1}}^{*}\left(L_{\boldsymbol{d}}^{\infty}\right), i_{\boldsymbol{d}, \boldsymbol{d}^{\prime}}\right)$, where $H_{\mathbb{T} \times S^{1}}^{*}\left(L_{\boldsymbol{d}}^{\infty}\right)=\mathbb{C}[P, \hbar, \lambda], i_{\boldsymbol{d}, \boldsymbol{d}^{\prime}}(\alpha)=$ $\alpha \prod_{i=1}^{N} \prod_{k=\left\langle w_{i}, \boldsymbol{d}^{\prime}\right\rangle}^{\left\langle w_{i}, \boldsymbol{d}\right\rangle-1} W_{i, k}$ and $W_{i, k}=\sum_{a=1}^{r} m_{i a} P_{a}-\lambda_{i}-k \hbar$. It has an action of $\mathbb{C}\left\langle P, Q^{ \pm}, \hbar, \lambda\right\rangle$. Let $\Delta^{\mathbb{T}}$ be the image of 1 in $H_{\mathbb{T} \times S^{1}}^{*}\left(L_{0}^{\infty}\right)$. Let $F H_{0}^{\mathbb{T}}$ be a $\mathbb{C}\langle P, Q, \hbar, \lambda\rangle$-submodule of $H_{\mathbb{T} \times S^{1}}^{\infty / 2}$ generated by $\Delta^{\mathbb{T}}$. Finally, we define $F H_{\mathbb{T} \times S^{1}}^{*}$ as the $Q$-adic completion of $F H_{0}^{\mathbb{T}}$. We can check that (by the argument in [16]) $F H_{\mathbb{T} \times S^{1}}^{*}$ is a free module over $\mathbb{C}[\hbar, \lambda] \llbracket Q \rrbracket$ and has $\left\{T_{i}(P) \Delta^{\mathbb{T}}\right\}_{i=0}^{s}$ as a basis, where $T_{i}\left(y_{1}, \ldots, y_{r}\right)$ is the polynomial in Proposition 5.5. By the same argument as Proposition 5.2, 5.4, we obtain

$$
H_{\mathbb{T} \times S^{1}}^{\infty / 2} \cong M_{\text {Mell }}^{\mathbb{T}}, \quad F H_{0}^{\mathbb{T}} \cong \mathbb{C}\langle q, \hbar \partial, \hbar, \lambda\rangle / I_{\text {poly }}^{\mathbb{T}}, \quad F H_{\mathbb{T} \times S^{1}}^{*} \cong \mathbb{C}[\hbar, \lambda] \llbracket q \rrbracket\langle\hbar \partial\rangle / \overline{I_{\text {poly }}^{\mathbb{T}}},
$$

where $I_{\text {poly }}^{\mathbb{T}}=I_{\text {Mell }}^{\mathbb{T}} \cap \mathbb{C}\langle q, \hbar \partial, \hbar, \lambda\rangle$ and $\overline{I_{\text {poly }}^{\mathbb{T}}}$ is the closure in the $q$-adic topology. The relationship between the $\mathbb{T} \times S^{1}$-equivariant Floer cohomology and $\mathbb{T}$-equivariant quantum cohomology is given as follows (c.f. Theorem [5.6).

Theorem 6.4. There exists an embedding $\mathfrak{e m b}:\left(\mathbb{C}^{r}, 0\right) \rightarrow\left(\mathbb{C}^{s+1}, 0\right)$ and an isomorphism of $D$-modules $\Phi_{\mathfrak{e m b}}: \mathfrak{e m b}^{*}\left(Q D M_{\mathbb{T}}^{*}(X)\right) \cong F H_{\mathbb{T} \times S^{1}}^{*}$. The map $\mathfrak{e m b}$ is given by the equations

$\hat{t}_{0}=F_{0}(q ; \lambda), \hat{q}_{1}=q_{1} \exp \left(F_{1}(q ; \lambda)\right), \ldots, \hat{q}_{r}=q_{r} \exp \left(F_{r}(q ; \lambda)\right), \hat{t}_{r+1}=F_{r+1}(q ; \lambda), \ldots, \hat{t}_{s}=F_{s}(q ; \lambda)$ for $F_{i}(q ; \lambda) \in \mathbb{C}[\lambda] \llbracket q \rrbracket, F_{i}(0 ; \lambda)=0$ and $\left.\Phi_{\mathfrak{e m b}}\right|_{q=0}$ is determined by the canonical isomorphism $H_{\mathbb{T}}^{*}(X) \otimes \mathbb{C}[\hbar] \cong F H_{\mathbb{T} \times S^{1}}^{*} / \sum_{a=1}^{r} q_{a} F H_{\mathbb{T} \times S^{1}}^{*}$.

This theorem is a generalization of the result of [17] to the $\mathbb{T}$-equivariant case. The proof is completely similar and based on a $\mathbb{T}$-equivariant version of Coates-Givental's quantum Lefschetz theorem [4]. The main points in the proof are (1) any toric variety can be realized as a complete intersection of torus invariant divisors in a Fano toric variety $\widetilde{X},(2)$ the $J$-function of $F H_{\mathbb{T} \times S^{1}}^{*}$ is identical with the Coates-Givental modification of the $J$-function of small quantum cohomology of the ambient $\widetilde{X}$, and (3) the reconstruction from small to big is unique. We can see that Coates-Givental's quantum Lefschetz theorem admits a $\mathbb{T}$-equivariant generalization. 
6.4. Asymptotic solution via equivariant mirror. Using the equivariant mirror, we will construct asymptotic solutions subject to the classical limit condition (36). Then it follows that the $R$-conjecture 6.2 holds for toric varieties. Unfortunately, we could not prove the convergence of $\mathbb{T}$-equivariant quantum cohomology using the method in this paper. Instead, we use a result in a forthcoming paper [18, which proves the convergence of equivariant quantum cohomology by the localization method.

We start with the construction of canonical coordinates for equivariant mirror. Each fixed point $\sigma \in X^{\mathbb{T}}$ can be written as an intersection of $N$ toric divisors $\cap_{i \in I_{\sigma}} D_{i}$, where $I_{\sigma}$ is a subset of $\{1,2, \ldots, r+N\}$. We can use $\left\{\mathrm{w}_{i}\right\}_{i \in I_{\sigma}}$ as fiber coordinates of the family $\pi: Y \rightarrow\left(\mathbb{C}^{*}\right)^{r}$. We write for $j \notin I_{\sigma}$,

$$
\mathrm{w}_{j}=\prod_{a=1}^{r} q_{a}^{l_{a j}^{\sigma}} \prod_{i \in I_{\sigma}} \mathrm{w}_{i}^{x_{j i}^{\sigma}} .
$$

Here, the matrix $l_{a j}^{\sigma}$ is the inverse of $\left(m_{j a}\right)_{j \notin I^{\sigma}, a=1, \ldots, r}$ and $x_{j i}^{\sigma}=-\sum_{a=1}^{r} m_{i a} l_{a j}^{\sigma}$. Since $\left\{p_{a}\right\}_{a=1}^{r}$ is a nef basis, it follows that the matrix $l_{a j}^{\sigma}$ satisfies $l_{a j}^{\sigma} \geq 0$ and $\sum_{a=1}^{r} l_{a j}^{\sigma}>0$.

We need the following elementary lemma. See the Appendix for the proof.

Lemma 6.5. Let $U \subset \mathbb{C}^{l}$ be a neighborhood of 0 and $D \subset U \backslash\{0\}$ be the complement of an analytic subvariety in $U$. Let $f\left(q_{1}, \ldots, q_{r}, \lambda_{1}, \ldots, \lambda_{l}\right)$ be a function holomorphic in the neighborhood of $\{q=0, \lambda \in D\}$. Assume that $f(\cdot, \lambda)$ can be expanded in the form $\sum_{\boldsymbol{d} \geq 0} f_{\boldsymbol{d}}(\lambda) q^{\boldsymbol{d}}$ for each $\lambda \in D$ and that $f_{\boldsymbol{d}}(\lambda)$ can be analytically continued to a holomorphic function on $U$. Then $f$ can be extended to a holomorphic function around $q=\lambda=0$.

Put $\chi_{i}(\sigma):=-w_{i}(\sigma)$. Then we have the following relations.

$$
\begin{gathered}
T_{\sigma}^{*} X \cong \bigoplus_{i \in I_{\sigma}} \chi_{i}(\sigma), \quad \chi_{i}(\sigma)=\lambda_{i}+\sum_{j \notin I_{\sigma}} \lambda_{j} x_{j i}^{\sigma} \quad \text { for } i \in I_{\sigma}, \\
\chi_{j}(\sigma)=\lambda_{j}-\sum_{a=1}^{r} m_{j a} p_{a}(\sigma)=0 \quad \text { for } j \notin I_{\sigma},
\end{gathered}
$$

Lemma 6.6. (i) For each $\sigma \in X^{\mathbb{T}}$, there exists a unique branch of critical points $\left\{\operatorname{crit}_{\sigma}(q)\right\}_{q}$ of $F_{q}^{\mathbb{T}}$ such that in a coordinate system $\left\{\mathrm{w}_{i}\right\}_{i \in I_{\sigma}}$,

$$
\mathrm{w}_{i}\left(\operatorname{crit}_{\sigma}(q)\right)=-\chi_{i}(\sigma)+O(q), \quad i \in I_{\sigma} .
$$

Here, $O(q)$ has an expansion of the form $\sum_{|\boldsymbol{d}|>0} f_{\boldsymbol{d}}(\lambda) q^{\boldsymbol{d}}$ for $f_{\boldsymbol{d}}(\lambda) \in \mathbb{C}\left[\lambda, \chi_{i}(\sigma)^{-1} ; i \in I_{\sigma}\right]$.

(ii) The critical value $F_{q}^{\mathbb{T}}\left(\operatorname{crit}_{q}(\sigma)\right)$ equals the pull-back $\mathfrak{e m b}^{*}\left(\mathbf{u}_{\sigma}\right)$ of the canonical coordinate satisfying the classical limit condition (34), where $\mathfrak{e m b}$ is the map in Theorem 6.4.

(iii) The defining equations of the map $\mathfrak{c m b}$ are convergent power series.

Proof. (i) follows from a direct calculation. For (ii), first note that there exists an isomorphism

$$
d F^{\mathbb{T}}: \bigcup_{q \in\left(\mathbb{C}^{*}\right)^{r}} \operatorname{Crit}\left(F_{q}^{\mathbb{T}}\right) \cong \operatorname{Ch}\left(M_{\text {Mell }}^{\mathbb{T}}\right)
$$

as in non-equivariant case (Lemma [5.10). Because we have a surjection

$$
M_{\text {Mell }}^{\mathbb{T}} \otimes_{\mathbb{C}\left[\hbar, \lambda, q^{ \pm}\right]} \mathbb{C}[\hbar, \lambda] \llbracket q \rrbracket\left[q^{-1}\right] \rightarrow F H_{\mathbb{T} \times S^{1}}^{*}\left[q^{-1}\right] \cong \mathfrak{e m b ^ { * }}\left(Q D M_{\mathbb{T}}^{*}(X)\right)\left[q^{-1}\right],
$$

$\left.\mathfrak{e m b}^{*}\left(\operatorname{Ch}\left(Q D M_{\mathbb{T}}^{*}(X)\right)\right)\right|_{q_{a} \neq 0}$ is embedded in $\bigcup_{q} \operatorname{Crit}\left(F_{q}^{\mathbb{T}}\right)$. The characteristic variety of $Q D M_{\mathbb{T}}^{*}(X)$ is the union $\bigcup_{\sigma} \operatorname{Graph}\left(d \mathrm{u}_{\sigma}\right)$ of graphs of the differentials of canonical coordinates. Thus, for a branch $\{\operatorname{crit}(q)\}$ contained in $\mathfrak{e m b}^{*}\left(\mathrm{Ch}\left(Q D M_{\mathbb{T}}^{*}(X)\right)\right)$, the critical value $F_{q}^{\mathbb{T}}(\operatorname{crit}(q))$ gives us a pull-back of a canonical coordinate. Furthermore, by the relations (37), it follows that 
$F_{q}^{\mathbb{T}}\left(\operatorname{crit}_{\sigma}(q)\right)$ satisfies the classical limit condition (34). From this we can see that the branch $\left\{\operatorname{crit}_{\sigma}(q)\right\}$ actually corresponds to a branch of $\operatorname{Ch}\left(Q D M_{\mathbb{T}}^{*}(X)\right)$ and $F_{q}^{\mathbb{T}}\left(\operatorname{crit}_{\sigma}(q)\right)=\mathfrak{e m b}^{*}\left(\mathbf{u}_{\sigma}\right)(q)$. In the forthcoming paper [18, we will prove that $Q H_{\mathbb{T}}^{*}(X)$ has convergent structure constants. Thus, the natural flat coordinates $\hat{x}=\left\{\hat{t}_{0}, \hat{q}_{1}, \ldots, \hat{q}_{r}, \hat{t}_{r+1}, \ldots, \hat{t}_{s}\right\}$ of $Q H_{\mathbb{T}}^{*}(X)$ can be written as analytic functions in $\mathrm{u}_{\sigma}$ and $\lambda$. Because $F_{q}^{\mathbb{T}}\left(\operatorname{crit}_{\sigma}(q)\right)$ is a multi-valued analytic function in $q$ and $\lambda, \mathfrak{e m b}^{*}\left(\hat{x}_{i}\right)$ is a (possibly multi-valued) analytic function in $q$ and $\lambda$. It is easy to see that it is holomorphic in the neighborhood of $\{q=0, \lambda$ generic $\}$ (identically zero at $q=0$ ). Since $\mathfrak{e m b}^{*}\left(\hat{x}_{i}\right)$ belongs to $\mathbb{C}[\lambda] \llbracket q \rrbracket$, (iii) follows from Lemma 6.5.

Remark 6.7. (i) The above lemma shows that Conjecture 6.1 holds for toric varieties.

(ii) The branch $\left\{\operatorname{crit}_{\sigma}(q)\right\}_{q}$ in the above lemma corresponds to a branch described in Proposition 5.11 (ii) in the non-equivariant limit.

(iii) Because the map $\mathfrak{e m b}$ preserves the degree, the homogeneity for $\mathfrak{e m b}^{*}\left(\mathbf{u}_{\sigma}\right)=F_{q}^{\mathbb{T}}\left(\operatorname{crit}_{\sigma}(q)\right)$ can be written as $c_{0}(\lambda)+\left(\sum_{a=1}^{r} c_{a} q_{a} \partial / \partial q_{a}+\sum_{j=1}^{r+N} \lambda_{j} \partial / \partial \lambda_{j}\right) F_{q}^{\mathbb{T}}\left(\operatorname{crit}_{\sigma}(q)\right)=F_{q}^{\mathbb{T}}\left(\operatorname{crit}_{\sigma}(q)\right)$ for $c_{0}(\lambda)=-\sum_{j=1}^{r+N} \lambda_{j}$. This can also be shown by a direct calculation.

Let $\Gamma(\sigma)$ be the descending Morse cycle of $\Re\left(F_{q}^{\mathbb{T}} / \hbar\right)$ from the critical point $\operatorname{crit}_{\sigma}(q)$. From now, we write $\mathbf{u}_{\sigma}$ for $\mathfrak{e m b}^{*}\left(\mathbf{u}_{\sigma}\right)$ by abuse of notation. Let $\mathcal{I}_{\sigma}(q, \lambda, \hbar):=\mathcal{I}_{\Gamma(\sigma)}(q, \lambda, \hbar)$ be the oscillatory integral over $\Gamma(\sigma)$. By the method of stationary phase, $\mathcal{I}_{\sigma}$ can be expanded in an asymptotic series $\mathcal{I}_{\sigma}^{\text {asym }}$. Put $\mathrm{w}_{i}=e^{\mathrm{T}_{i}} \mathrm{w}_{i}\left(\operatorname{crit}_{\sigma}\right)$ and $\mathrm{T}_{i}=\sqrt{\hbar} \mathrm{t}_{i}$. Note that $\mathrm{T}_{j}=\sum_{i \in I_{\sigma}} x_{j i}^{\sigma} \mathrm{T}_{i}$ for $j \notin I_{\sigma}$.

$$
\begin{aligned}
& \mathcal{I}_{\sigma}(q, \lambda, \hbar)=e^{F_{q}^{\mathbb{T}}\left(\operatorname{crit}_{\sigma}(q)\right) / \hbar} \int_{\Gamma(\sigma)} \exp \frac{F_{q}^{\mathbb{T}}\left(e^{\top} \operatorname{crit}_{\sigma}(q)\right)-F_{q}^{\mathbb{T}}\left(\operatorname{crit}_{\sigma}(q)\right)}{\hbar} \prod_{i \in I_{\sigma}} d \mathrm{~T}_{i} \\
&=e^{\mathbf{u}_{\sigma} / \hbar} \int_{\Gamma(\sigma)} \exp \frac{\sum_{i=1}^{r+N}\left(e^{\mathrm{T}_{i}}-1\right) \mathrm{w}_{i}\left(\operatorname{crit}_{\sigma}(q)\right)+\lambda_{i} \mathrm{~T}_{i}}{\hbar} \prod_{i \in I_{\sigma}} d \mathrm{~T}_{i} \\
& \sim \hbar^{N / 2} e^{\mathrm{u}_{\sigma} / \hbar} \int_{\mathbb{R}^{N}} e^{\sum_{i=1}^{r+N} \mathrm{w}_{i}\left(\operatorname{crit}_{\sigma}\right) \mathbf{t}_{i}^{2} / 2} \exp \left(\sum_{i=1}^{r+N} \sum_{n=3}^{\infty} \hbar^{n / 2-1} \mathbf{w}_{i}\left(\operatorname{crit}_{\sigma}\right) \frac{\mathrm{t}_{i}^{n}}{n !}\right) \prod_{i \in I_{\sigma}} d \mathbf{t}_{i} \\
&=: \mathcal{I}_{\sigma}^{\operatorname{asym}}(q, \lambda, \hbar)=(2 \pi \hbar)^{N / 2} e^{\mathbf{u}_{\sigma} / \hbar} \frac{1}{\sqrt{ \pm \operatorname{Hess}_{\sigma}}} \sum_{n=0}^{\infty} \mathcal{I}_{\sigma n}(q, \lambda) \hbar^{n} .
\end{aligned}
$$

Here, $\mathcal{I}_{\sigma 0}=1, \pm \operatorname{Hess}_{\sigma}=(-1)^{N} \operatorname{det}\left(\partial^{2} F_{q}^{\mathbb{T}} / \partial \mathrm{T}_{i} \partial \mathrm{T}_{j}\left(\operatorname{crit}_{\sigma}(q)\right)\right)_{i, j \in I_{\sigma}}$ and we assumed $\mathrm{w}_{i}\left(\operatorname{crit}_{\sigma}\right)<$ 0 and $\hbar>0$. All half-integer powers of $\hbar$ disappear due to the antisymmetry. We can see that the analytic function $\mathcal{I}_{\sigma n}(q, \lambda)$ can be expanded in a $q$-series of the form $\sum_{\boldsymbol{d}} f_{\boldsymbol{d}}(\lambda) q^{\boldsymbol{d}}$, where $f_{\boldsymbol{d}}(\lambda) \in \mathbb{C}\left[\lambda, \chi_{i}(\sigma)^{-1} ; i \in I_{\sigma}\right]$.

Proposition 6.8. The asymptotic series $\mathcal{I}_{\sigma}^{\text {asym }}$ is a formal solution to the $D$-module $F H_{\mathbb{T} \times S^{1}}^{*}$ for each $\sigma \in X^{\mathbb{T}}$. More precisely, for any differential operator $f(q, \lambda, \hbar \partial, \hbar) \in \overline{I_{\text {poly }}^{\mathbb{T}}} \subset \mathbb{C}[\hbar, \lambda] \llbracket q \rrbracket\langle\hbar \partial\rangle$, we have $f(q, \lambda, \hbar \partial, \hbar) \mathcal{I}_{\sigma}^{\text {asym }}(q, \lambda, \hbar)=0$ as an $\hbar$-series.

Proof. Take $f \in \overline{I_{\text {poly }}^{\mathbb{T}}}$. We put $f \cdot \mathcal{I}_{\sigma}^{\text {asym }}=(2 \pi \hbar)^{N / 2} e^{\mathbf{u}_{\sigma} / \hbar} \sum_{k=0}^{\infty} a_{k}(q, \lambda) \hbar^{k}$. Note that $a_{k}(q, \lambda)$ is a formal power series in $q$. Let $f_{n} \in I_{\text {poly }}^{\mathbb{T}}$ be a sequence converging to $f \in \overline{I_{\text {poly }}^{\mathbb{T}}}$ such that $g_{n}=f-f_{n}=O\left(q^{n}\right)$. Then we have

$$
g_{n} \cdot \mathcal{I}_{\sigma}^{\text {asym }}=f \cdot \mathcal{I}_{\sigma}^{\text {asym }}=(2 \pi \hbar)^{N / 2} e^{\mathbf{u}_{\sigma} / \hbar} \sum_{k=0}^{\infty} a_{k}(q, \lambda) \hbar^{k}
$$


by Proposition 6.3. Because we have the following expansions

$$
\left( \pm \operatorname{Hess}_{\sigma}\right)^{-1 / 2}=\left(\prod_{i \in I_{\sigma}} \chi_{i}(\sigma)\right)^{-1 / 2}\left(1+\sum_{|\boldsymbol{d}|>0} b_{\boldsymbol{d}}(\lambda) q^{\boldsymbol{d}}\right), \quad q_{a} \frac{\partial \mathrm{u}_{\sigma}}{\partial q_{a}}=\sum_{\boldsymbol{d} \geq 0} c_{\boldsymbol{d}}(\lambda) q^{\boldsymbol{d}}
$$

for some $b_{\boldsymbol{d}}(\lambda), c_{\boldsymbol{d}}(\lambda) \in \mathbb{C}\left[\lambda, \chi_{i}(\sigma)^{-1} ; i \in I_{\sigma}\right]$, we can see from (39) that $a_{k}(q, \lambda)=O\left(q^{n}\right)$. Because this holds for all $n, a_{k}$ must be zero.

In non-equivariant case, each coefficient of the asymptotic expansion of $\mathcal{I}_{\Gamma}$ in $\hbar$ is still analytic function in $q$ but not necessarily expanded in $q$-series. However, $f(q, \hbar \partial, \hbar) \cdot \mathcal{I}_{\Gamma}^{\operatorname{asym}}(q, \hbar)$ does make sense for $f \in \mathcal{O}_{\text {small }}^{\hbar}\langle\hbar \partial\rangle$ as an $\hbar$-series.

Corollary 6.9. Let $\Gamma$ be the descending Morse cycle of $\Re\left(F_{q}\right)$ from a critical point in a branch described in Proposition 5.11 (ii). The asymptotic expansion of the non-equivariant mirror oscillatory integral $\mathcal{I}_{\Gamma}(q, \hbar)$ in $\hbar$ gives a formal solution to $\widetilde{F H}_{S^{1}}^{*}$. More precisely, any differential operator in $\overline{I_{\text {poly }}} \subset \mathcal{O}_{\text {small }}^{\hbar}\langle\hbar \partial\rangle$ annihilates the asymptotic expansion of $\mathcal{I}_{\Gamma}$ as an $\hbar$-series.

For $v \in Q H_{\mathbb{T}}^{*}(X)$, there exists a differential operator $\mathcal{D}[v](q, \lambda, \hbar \partial, \hbar) \in \mathbb{C}[\hbar, \lambda] \llbracket q \rrbracket\langle\hbar \partial\rangle$ such that $\Phi_{\mathfrak{e m b}}(v)=\mathcal{D}[v](Q, \lambda, P, \hbar) \Delta^{\mathbb{T}}$. We can write $v=\mathcal{D}[v]\left(q, \lambda, \nabla^{\hbar}, \hbar\right) \cdot \Phi_{\mathfrak{e m b}}^{-1} \Delta^{\mathbb{T}}$, where $\nabla_{a}^{\hbar}=$ $\nabla_{\mathfrak{e m b}_{*}\left(\partial_{a}\right)}^{\hbar}$. Define a formal section $s_{\sigma}(q, \lambda, \hbar)$ of $\mathfrak{e m} \mathfrak{b}^{*} \mathcal{T}_{\mathcal{M}}$ by the formula

$$
\left\langle v,(-2 \pi \hbar)^{N / 2} s_{\sigma}\right\rangle_{\mathbb{T}}=\mathcal{D}[v](q, \lambda, \hbar \partial, \hbar) \cdot \mathcal{I}_{\sigma}^{\text {asym }} .
$$

By Proposition 6.8, the right hand side depends only on $v$ and not on a choice of $\mathcal{D}[v]$. Using $\left\langle\partial / \partial \mathbf{u}_{\sigma}, \partial / \partial \mathbf{u}_{\tau}\right\rangle_{\mathbb{T}}=\delta_{\sigma \tau} \triangle_{\sigma}^{-2}$, we can also write

$$
s_{\sigma}(q, \lambda, \hbar)=\frac{1}{(-2 \pi \hbar)^{N / 2}} \sum_{\tau} \triangle_{\tau}\left(\mathcal{D}\left[\psi_{\tau}(q)\right] \mathcal{I}_{\sigma}^{\text {asym }}\right) \triangle_{\tau} \frac{\partial}{\partial \mathrm{u}_{\tau}},
$$

where $\psi_{\tau}(q)=\psi_{\tau}(\mathfrak{e m b}(q))$.

Theorem 6.10. (i) The section $s_{\sigma}$ is the pull-back of an asymptotic solution in section [6.1 by the map $\mathfrak{e m b}$.

(ii) This satisfies the classical limit condition (36).

(iii) The $R$-conjecture 6.2 holds for smooth projective toric variety.

Proof. (i): For $v \in H_{\mathbb{T}}^{*}(X)$ and $\tilde{s}_{\sigma}=(-2 \pi \hbar)^{N / 2} s_{\sigma}$, we have

$$
\begin{aligned}
\left\langle v, \hbar q_{a} \frac{\partial}{\partial q_{a}} \tilde{s}_{\sigma}\right\rangle_{\mathbb{T}} & =\hbar \partial_{a} \mathcal{D}[v] \mathcal{I}_{\sigma}^{\text {asym }}=\mathcal{D}\left[\nabla_{\mathfrak{e m b} \mathfrak{b}_{*}\left(\partial_{a}\right)}^{\hbar} v\right] \mathcal{I}_{\sigma}^{\text {asym }} \\
& =\left\langle\mathfrak{e m b}_{*}\left(\partial_{a}\right) * v, \tilde{s}_{\sigma}\right\rangle_{\mathbb{T}}=\left\langle v, \mathfrak{e m b}_{*}\left(\partial_{a}\right) * \tilde{s}_{\sigma}\right\rangle_{\mathbb{T}} .
\end{aligned}
$$

Therefore, $s_{\sigma}$ is a solution to the differential equation (35). We calculate

$$
\mathcal{D}\left[\psi_{\tau}(q)\right] \mathcal{I}_{\sigma}^{\text {asym }}=(2 \pi \hbar)^{N / 2} e^{\mathbf{u}_{\sigma} / \hbar}\left(\frac{\mathcal{D}\left[\psi_{\tau}(q)\right]\left(q, \lambda, \partial \mathbf{u}_{\sigma}, \hbar=0\right)}{\sqrt{ \pm \operatorname{Hess}_{\sigma}}}+O(\hbar)\right) .
$$

Let $V(q, \lambda, \hbar \partial, \hbar)$ be a differential operator such that $V\left(q, \lambda, \nabla^{\hbar}, \hbar\right) \cdot 1=\Phi_{\mathfrak{e m b}}^{-1} \Delta^{\mathbb{T}}$. Then we have $\psi_{\tau}(q)=\mathcal{D}\left[\psi_{\tau}(q)\right]\left(q, \lambda, \nabla^{\hbar}, \hbar\right) V\left(q, \lambda, \nabla^{\hbar}, \hbar\right) \cdot 1=\mathcal{D}\left[\psi_{\tau}(q)\right](q, \lambda, \partial *, 0) V(q, \lambda, \partial *, 0) 1$, where $\partial_{a} *$ is the quantum product by $\mathfrak{e m b}_{*}\left(\partial_{a}\right)$. By using (33), we have

$$
\begin{aligned}
\delta_{\sigma \tau} \psi_{\sigma}(q)=\psi_{\tau}(q) * \psi_{\sigma}(q) & =\mathcal{D}\left[\psi_{\tau}(q)\right](q, \lambda, \partial *, 0) V(q, \lambda, \partial *, 0) \psi_{\sigma}(q) \\
& =\mathcal{D}\left[\psi_{\tau}(q)\right]\left(q, \lambda, \partial \mathbf{u}_{\sigma}, 0\right) V\left(q, \lambda, \partial \mathbf{u}_{\sigma}, 0\right) \psi_{\sigma}(q)
\end{aligned}
$$


Therefore,

$$
\frac{1}{(-2 \pi \hbar)^{N / 2}} \triangle_{\tau} \mathcal{D}\left[\psi_{\tau}(q)\right] \mathcal{I}_{\sigma}^{\mathrm{asym}}=e^{\mathbf{u}_{\sigma} / \hbar}\left(\frac{\delta_{\sigma \tau} \triangle_{\sigma}}{V\left(q, \lambda, \partial \mathbf{u}_{\sigma}, 0\right) \sqrt{\mathrm{Hess}_{\sigma}}}+O(\hbar)\right) .
$$

The equality $\triangle_{\sigma}=V\left(q, \lambda, \partial \mathbf{u}_{\sigma}, 0\right) \sqrt{\operatorname{Hess}_{\sigma}}$ follows from the differential equation $\hbar \partial_{a} s_{\sigma}=\partial_{a} * s_{\sigma}$,

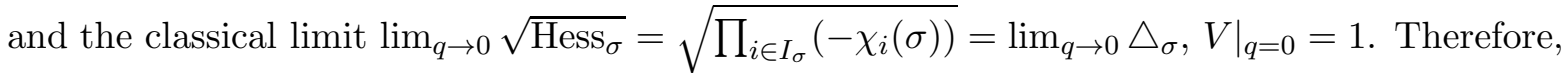
$s_{\sigma}$ is an asymptotic solution in section 6.1.

(ii): By Lemma [6.6] and (38), we have

$$
\begin{aligned}
\triangle_{\tau} \mathcal{D}\left[\psi_{\tau}(q)\right] \mathcal{I}_{\sigma}=\triangle_{\tau} \mathcal{D}\left[\psi_{\tau}(q)\right]\left(e^{\mathbf{u}_{\sigma} / \hbar} \int_{\mathbb{R}_{\geq 0}^{N}} e^{-\sum_{i \in I_{\sigma}} \chi_{i}(\sigma)\left(\mathrm{x}_{i}-\log \mathrm{x}_{i}-1\right) / \hbar+O(q)} \prod_{i \in I_{\sigma}} d \log \mathrm{x}_{i}\right) \\
=e^{\mathbf{u}_{\sigma} / \hbar} \int_{\mathbb{R}_{\geq 0}^{N}}\left(\triangle_{\tau} \mathcal{D}\left[\psi_{\tau}(q)\right]\left(0, \lambda, \partial \mathbf{u}_{\sigma}, \hbar\right) \prod_{i \in I_{\sigma}} e^{-\left(\mathrm{x}_{i}-1\right) \chi_{i}(\sigma) / \hbar} \mathbf{x}_{i}^{\chi_{i}(\sigma) / \hbar-1}+O(q)\right) \prod_{i \in I_{\sigma}} d \mathrm{x}_{i} \\
=e^{\mathbf{u}_{\sigma} / \hbar}\left(\delta_{\sigma \tau} \sqrt{\prod_{i \in I_{\sigma}}-\chi_{i}(\sigma)} \prod_{i \in I_{\sigma}} e^{\chi_{i}(\sigma) / \hbar}\left(\frac{\chi_{i}(\sigma)}{\hbar}\right)^{-\chi_{i}(\sigma) / \hbar} \Gamma\left(\frac{\chi_{i}(\sigma)}{\hbar}\right)+O(q)\right) .
\end{aligned}
$$

We put $\mathrm{x}_{i}=e^{\mathrm{T}_{i}}$ in the first line, used $\partial_{a} \partial_{b} \mathbf{u}_{\sigma}=O(q)$ in the second line and $\mathcal{D}\left[\psi_{\tau}(q)\right]\left(0, \lambda, \partial \mathbf{u}_{\sigma}, \hbar\right)=$ $\delta_{\tau \sigma}$ in the third line. Note that we are assuming $\chi_{i}(\sigma)>0$. The conclusion follows from the asymptotic expansion of the Gamma function around infinity (Stirling's formula).

$$
\log \Gamma(z) \sim\left(z-\frac{1}{2}\right) \log z-z+\frac{1}{2} \log 2 \pi+\sum_{k=1}^{\infty} \frac{B_{2 k}}{2 k(2 k-1) z^{2 k-1}}, \quad \Re(z)>0 .
$$

(iii): First note that if the asymptotic solution is regular at $\lambda=0$ for one semisimple point $t_{0}$, then it is regular for any semisimple point. (We can use the value at $t_{0}$ as an initial condition for the differential equation (35).) Thus, it suffices to check that the above solution $s_{\sigma}$ is regular at $\lambda=0$ for a semisimple $q$. Let $T_{i}\left(y_{1}, \ldots, y_{r}\right)$ be the polynomial in Proposition 5.5. Because $\left\{T_{i}(P) \Delta^{\mathbb{T}}\right\}_{i=0}^{s}$ forms a $\mathbb{C}[\hbar, \lambda] \llbracket q \rrbracket$-basis of $F H_{\mathbb{T} \times S^{1}}^{*}$, we can write $\Phi_{\mathfrak{e m b}}\left(\partial / \partial t_{i}\right)=$ $\sum_{j=0}^{s} A_{i j}(q, \lambda, \hbar) T_{j}(P) \Delta^{\mathbb{T}}$ for some $A_{i j} \in \mathbb{C}[\hbar, \lambda] \llbracket q \rrbracket$. Let $\triangle_{\tau} \psi_{\tau}(q)=\sum_{i=0}^{s} \Psi_{\tau i}(q, \lambda)\left(\partial / \partial t_{i}\right)$. Because $Q H_{\mathbb{T}}^{*}(X)$ is convergent, $\Psi_{\tau i}(q, \lambda)$ is an analytic function of $q, \lambda$ which is regular at $\lambda=0$ for semisimple $q$. We can take $\mathcal{D}\left[\psi_{\tau}(q)\right]$ as

$$
\triangle_{\tau} \mathcal{D}\left[\psi_{\tau}(q)\right]=\sum_{i, j=0}^{s} \Psi_{\tau i}(q, \lambda) A_{i j}(q, \lambda, \hbar) T_{j}(\hbar \partial)
$$

Then, the matrix $R$ is given by $(-2 \pi \hbar)^{N / 2} e^{\mathbf{u}_{\sigma} / \hbar} R_{\tau \sigma}=\sum_{i, j=0}^{s} \Psi_{\tau i}(q, \lambda) A_{i j}(q, \lambda, \hbar) T_{j}(\hbar \partial) \mathcal{I}_{\sigma}^{\text {asym }}$. Because we can write $T_{j}(\hbar \partial) \mathcal{I}_{\sigma}^{\text {asym }}=(-2 \pi \hbar)^{N / 2} \mathcal{R}_{j \sigma} e^{\mathbf{u}_{\sigma} / \hbar}$ for some $\mathcal{R}_{j \sigma}=\sum_{n \geq 0} \mathcal{R}_{j \sigma}^{(n)}(q, \lambda) \hbar^{n}$, we have

$$
R_{\tau \sigma}(q, \lambda, \hbar)=\sum_{i, j=0}^{s} \Psi_{\tau i}(q, \lambda) A_{i j}(q, \lambda, \hbar) \mathcal{R}_{j \sigma}(q, \lambda, \hbar) .
$$

On the right hand side, the regularity at $\lambda=0$ is clear for $\Psi_{\tau i}$ and $\mathcal{R}_{j \sigma}$. Thus, it suffices to show that $A_{i j}^{(n)}(q, \lambda)$ is a holomorphic function around $q=\lambda=0$ where $A_{i j}=\sum_{n \geq 0} A_{i j}^{(n)}(q, \lambda) \hbar^{n}$. Since $\left(A_{i j}\right)=\left(\Psi_{\tau i}\right)^{-1}\left(R_{\tau \sigma}\right)\left(\mathcal{R}_{j \sigma}\right)^{-1}$ and $R_{\tau \sigma}^{(n)}(q, \lambda)$ is an analytic function (because $Q H_{\mathbb{T}}^{*}(X)$ is convergent), we can see that $A_{i j}^{(n)}(q, \lambda)$ is convergent around $q=\lambda=0$ from Lemma 6.5. 


\section{AppendiX}

7.1. Proof of Lemma 4.9, First we study $\varepsilon_{1}(M)$. We have

$$
\begin{aligned}
\varepsilon_{1}(d, M) & :=\sum_{i=1}^{d-1} \frac{(d+1)^{M}}{(d-i+1)^{M}(i+1)^{M}} \\
& \leq \int_{0}^{d} \frac{(d+1)^{M} d x}{(d-x+1)^{M}(x+1)^{M}}=\epsilon^{M-1}(1-\epsilon)^{M} \int_{\epsilon}^{1-\epsilon} \frac{d y}{y^{M}(1-y)^{M}},
\end{aligned}
$$

where we put $y=(x+1) /(d+2)$ and $\epsilon=1 /(d+2)$. Set $y=\sin ^{2}(\theta / 2)$ and $\epsilon=\sin ^{2}(\alpha / 2)$. Then we have

$$
\varepsilon_{1}(d, M) \leq 4 \cos ^{2}\left(\frac{\alpha}{2}\right)(\sin \alpha)^{2 M-2} \int_{\alpha}^{\pi / 2} \frac{d \theta}{(\sin \theta)^{2 M-1}}
$$

Because we can assume $d \geq 2$, we have $0<\alpha \leq \pi / 3$. Using the formula (see [15], 2.515)

$$
\int \frac{d \theta}{(\sin \theta)^{2 M-1}}=\frac{(2 M-3) ! !}{(2 M-2) ! !}\left\{-\sum_{r=0}^{M-2} \frac{(2 M-4-2 r) ! !}{(2 M-3-2 r) ! !} \frac{\cos \theta}{(\sin \theta)^{2 M-2-2 r}}+\log \left|\tan \frac{\theta}{2}\right|\right\},
$$

we have

$$
\begin{aligned}
\varepsilon_{1}(d, M) \leq & 4 \cos ^{2}\left(\frac{\alpha}{2}\right) \frac{(2 M-3) ! !}{(2 M-2) ! !}\left\{\sum_{r=0}^{M-2} \frac{(2 M-4-2 r) ! !}{(2 M-3-2 r) ! !} \cos \alpha(\sin \alpha)^{2 r}\right. \\
& \left.-(\sin \alpha)^{2 M-2} \log \left|\tan \frac{\alpha}{2}\right|\right\} \\
\leq & 4 \frac{(2 M-3) ! !}{(2 M-2) ! !}\left\{\frac{1}{1-\sin ^{2} \alpha}+(\sin \alpha)^{2 M-3} \frac{2}{e}\right\} \\
\leq & 4\left(1-\frac{1}{2 M-2}\right)\left(1-\frac{1}{2 M-4}\right) \cdots\left(1-\frac{1}{2}\right)\left(4+\frac{2}{e}\right)
\end{aligned}
$$

Therefore, we have $\varepsilon_{1}(M) \rightarrow 0$ as $M \rightarrow \infty$.

Next we study $\varepsilon_{2}\left(B_{2}, M\right)$. Set $C=B_{2} / B_{1}$. We have for $C>1$,

$$
\begin{aligned}
\sum_{i=1}^{d}\left(\frac{B_{1}}{B_{2}}\right)^{i} \frac{(d+1)^{M}}{(d+1-i)^{M}} & \leq \int_{0}^{d} \frac{(d+1)^{M} d x}{C^{x}(d+1-x)^{M}}+\frac{(d+1)^{M}}{C^{d}} \\
& \leq \int_{0}^{1-\frac{1}{d+1}} \frac{(d+1) d y}{C^{(d+1) y}(1-y)^{M}}+\left(\frac{2 M}{e \log C}\right)^{M} \\
& \leq \int_{0}^{1 / 2} \frac{(d+1) 2^{M}}{C^{(d+1) y}} d y+\int_{1 / 2}^{1-\frac{1}{d+1}} \frac{(d+1) d y}{C^{(d+1) / 2}(1-y)^{M}}+\left(\frac{2 M}{e \log C}\right)^{M} \\
& \leq \frac{2^{M}}{\log C}+\frac{(d+1)^{M}}{(M-1) C^{(d+1) / 2}}+\left(\frac{2 M}{e \log C}\right)^{M} \\
& \leq \frac{2^{M}}{\log C}+\frac{1}{M-1}\left(\frac{2 M}{e \log C}\right)^{M}+\left(\frac{2 M}{e \log C}\right)^{M}
\end{aligned}
$$

Hence we have $\varepsilon\left(B_{2}, N\right) \rightarrow 0$ as $B_{2} \rightarrow \infty$. 
7.2. Proof of Lemma 6.5. We prove the lemma by induction on $l$. We write $\lambda=\left(\lambda^{\prime}, \lambda_{l}\right) \in$ $\mathbb{C}^{l-1} \times \mathbb{C}$. Set $D^{\prime}=\left\{\lambda^{\prime} \in \mathbb{C}^{l-1} \mid\left\{\lambda^{\prime}\right\} \times \mathbb{C} \cap D\right.$ is non-empty $\}$. We will show that $f$ is holomorphic in the neighborhood of $\left\{q=0, \lambda_{l}=0, \lambda^{\prime} \in D^{\prime}\right\}$. We choose $\epsilon\left(\lambda^{\prime}\right)>0$ so that $\left\{\lambda^{\prime}\right\} \times\left\{\left|\lambda_{l}\right|=\right.$ $\left.\epsilon\left(\lambda^{\prime}\right)\right\} \subset D$. Then there exists a positive $C\left(\lambda^{\prime}\right)>0$ such that $\left|f_{\boldsymbol{d}}\left(\lambda^{\prime}, \lambda_{l}\right)\right| \leq C\left(\lambda^{\prime}\right)^{|\boldsymbol{d}|+1}$ on $\left|\lambda_{l}\right|=\epsilon\left(\lambda^{\prime}\right)$. For $|\zeta|<\epsilon\left(\lambda^{\prime}\right) / 2$, because $f_{\boldsymbol{d}}(\lambda)$ is holomorphic on $U$,

$$
f_{\boldsymbol{d}}\left(\lambda^{\prime}, \zeta\right)=\frac{1}{2 \pi i} \int_{|z|=\epsilon\left(\lambda^{\prime}\right)} \frac{f_{\boldsymbol{d}}\left(\lambda^{\prime}, z\right)}{z-\zeta} d z
$$

From this we can see that $f_{\boldsymbol{d}}\left(\lambda^{\prime}, \zeta\right) \leq 2 C\left(\lambda^{\prime}\right)^{|\boldsymbol{d}|+1}$ for $\lambda^{\prime} \in D^{\prime}$ and $|\zeta|<\epsilon\left(\lambda^{\prime}\right) / 2$.

\section{REFERENCES}

1. A. Bayer, Yuri I. Manin, (Semi)simple exercises in quantum cohomology. The Fano Conference, pp.143-173, Univ. Torino, Turin, 2004.

2. A. Bertram, Ionut Ciocan-Fontanine, Bumsig Kim, Two proofs of a conjecture of Hori and Vafa. Duke Math. J. 126 (2005), no. 1, pp.101-136.

3. A. Bertram, Ionut Ciocan-Fontanine, Bumsig Kim, Gromov-Witten invariants for abelian and non-abelian quotients. math.AG/0407254

4. T. Coates, A.B. Givental, Quantum Riemann - Roch, Lefschetz and Serre. math.AG/0110142

5. B. Dubrovin, Geometry of $2 D$ topological field theories. Integrable systems and quantum groups (Montecatini Terme, 1993), pp.120-348, Lecture Notes in Math. 1620, Springer, Berlin, 1996.

6. Tohru Eguchi, Kentaro Hori, Chuan-Sheng Xiong, Quantum cohomology and Virasoro algebra. Phys. Lett. B 402 (1997), no. 1-2, pp.71-80.

7. A. B. Givental, Homological geometry I. Projective hypersurfaces. Selecta Math. (N.S.) 1 (1995), no. 2, pp.325-345.

8. A. B. Givental, Homological geometry and mirror symmetry. In: Proceedings of the ICM, Zürich 1994, Birkhäuser 1995, vol 1, pp.472-480

9. A. B. Givental, Equivariant Gromov-Witten invariants. Internat. Math. Res. Notices 1996, no. 13, pp.613663.

10. A. B. Givental, A mirror theorem for toric complete intersections. Topological field theory, primitive forms and related topics (Kyoto, 1996), pp.141-175, Progr. Math., 160, Birkhäuser Boston, Boston, MA, 1998.

11. A. B. Givental, Elliptic Gromov-Witten invariants and the generalized mirror conjecture. Integrable systems and algebraic geometry, Proceedings of the Taniguchi symposium 1997, pp.107-155, World Scientific, 1998.

12. A. B. Givental Semisimple Frobenius structures at higher genus. Internat. Math. Res. Notices 2001, no. 23, pp. $1265-1286$.

13. A. B. Givental, Gromov-Witten invariants and quantization of quadratic Hamiltonians. Dedicated to the memory of I. G. Petrovskii on the occasion of his 100th anniversary. Mosc. Math. J. 1 (2001), no. 4, pp.551568,645 .

14. M. A. Guest, Quantum cohomology via D-modules. Topology 44 (2005), no. 2, pp.263-281.

15. I. S. Gradshteyn, I. M. Ryzhik, Table of integrals, series, and products. Fourth edition prepared by Ju. V. Geronimus and M. Ju. Cel̆tlin. Translated from the Russian by Scripta Technica, Inc. Translation edited by Alan Jeffrey, Academic Press, New York-London 1965.

16. H. Iritani, Quantum D-modules and equivariant Floer theory for free loop spaces., Math. Z. 252 (2006) no.3, pp. 577-622

17. H. Iritani, Quantum D-modules and generalized mirror transformations. math.DG/0411111

18. H. Iritani, Convergence of Gromov-Witten theory by localization. in preparation.

19. M. Jinzenji, Coordinate change of Gauss-Manin system and generalized mirror transformation. Internat. J. Modern Phys. A 20 (2005), no. 10, pp.2131-2156.

20. Dosang Joe, Bumsig Kim, Equivariant mirrors and the Virasoro conjecture for flag manifolds. Int. Math. Res. Not. 2003, no. 15, pp.859-882.

21. Yujiro Kawamata, Derived categories of toric varieties. math.AG/0503102

22. Bumsig Kim, A. Kresch, T. Pantev, Functoriality in intersection theory and a conjecture of Cox, Katz, and Lee. J. Pure Appl. Algebra 179 (2003), no. 1-2, pp.127-136.

23. A. G. Kushnirenko, Polyèdres de Newton et nombres de Milnor. Invent. Math. 32 (1976), no. 1, pp.1-31.

24. Yuri I. Manin, Frobenius manifolds, quantum cohomology, and moduli spaces. American Mathematical Society Colloquium Publications, 47. American Mathematical Society, Providence, RI, 1999. 
25. R. Pandharipande, Rational curves on hypersurfaces (after A. Givental). Séminaire Bourbaki. Vol. $1997 / 98$. Astérisque No. 252, (1998), Exp. No. 848, 5, pp.307-340.

Department of Mathematics, Graduate School of Science, Kyoto University, Oiwake-Cho, KiTASHIRAKAWA, SAKYO-KU, KYOTO, 606-8502, JAPAN.

E-mail address: iritani@math.kyoto-u.ac.jp 